ESAIM: M2AN 48 (2014) 1807-1857

DOI: $10.1051 / \mathrm{m} 2 \mathrm{an} / 2014021$
ESAIM: Mathematical Modelling and Numerical Analysis

www.esaim-m2an.org

\title{
ON SOME IMPLICIT AND SEMI-IMPLICIT STAGGERED SCHEMES FOR THE SHALLOW WATER AND EULER EQUATIONS
}

\author{
R. Herbin ${ }^{1}$, W. KheriJi ${ }^{2}$ AND J.-C. LATChÉ ${ }^{2}$
}

\begin{abstract}
In this paper, we propose implicit and semi-implicit in time finite volume schemes for the barotropic Euler equations (hence, as a particular case, for the shallow water equations) and for the full Euler equations, based on staggered discretizations. For structured meshes, we use the MAC finite volume scheme, and, for general mixed quadrangular/hexahedral and simplicial meshes, we use the discrete unknowns of the Rannacher-Turek or Crouzeix-Raviart finite elements. We first show that a solution to each of these schemes satisfies a discrete kinetic energy equation. In the barotropic case, a solution also satisfies a discrete elastic potential balance; integrating these equations over the domain readily yields discrete counterparts of the stability estimates which are known for the continuous problem. In the case of the full Euler equations, the scheme relies on the discretization of the internal energy balance equation, which offers two main advantages: first, we avoid the space discretization of the total energy, which involves cell-centered and face-centered variables; second, we obtain an algorithm which boils down to a usual pressure correction scheme in the incompressible limit. Consistency (in a weak sense) with the original total energy conservative equation is obtained thanks to corrective terms in the internal energy balance, designed to compensate numerical dissipation terms appearing in the discrete kinetic energy inequality. It is then shown in the 1D case, that, supposing the convergence of a sequence of solutions, the limit is an entropy weak solution of the continuous problem in the barotropic case, and a weak solution in the full Euler case. Finally, we present numerical results which confirm this theory.
\end{abstract}

Mathematics Subject Classification. 35Q31, 65N12, 76M10, 76M12.

Received January 1st, 2013. Revised January 16, 2014.

Published online October 10, 2014.

\section{INTRODUCTION}

The objective pursued in this work is to develop and analyze a class of efficient numerical schemes for the simulation of compressible flows at all Mach number regimes. To this purpose, our basic choice is to extend algorithms that are classical in the incompressible framework, namely pressure correction schemes based on (inf-sup stable) staggered discretizations.

\footnotetext{
Keywords and phrases. Finite volumes, finite elements, staggered, pressure correction, Euler equations, shallow-water equations, compressible flows, analysis.

1 Aix-Marseille Université, CNRS, Centrale Marseille, I2M, UMR 7373, 13453 Marseille, France. raphaele.herbin@univ-amu.fr

2 Institut de Radioprotection et de Sûreté Nucléaire (IRSN), BP 3, 13115 Saint-Paul-lez-Durance cedex, France.

kheriji.walid@gmail.com; jean-claude.latche@irsn.fr
} 
The fractional step strategy involving an elliptic pressure correction step has been recognized to yield algorithms which are not limited by stringent stability conditions (such as CFL conditions based on the celerity of the fastest waves) since the first attempts to build "all flow velocity" schemes in the late sixties [23] or in the early seventies [24]; these algorithms may be seen as an extension to the compressible case of the celebrated MAC scheme, introduced some years before [25]. These seminal papers have been the starting point for the development of numerous schemes, using staggered finite volume space discretizations $[4,6,34,35,38,41,47,64-69,71]$, colocated finite volumes $[2,10,32,33,36,37,39,43,48-51,54,57,59,61,70]$ or finite elements $[3,46,52,72]$. Algorithms proposed in these works may be essentially implicit-in-time, and the pressure correction step is then an ingredient of a SIMPLE-like iterative procedure, or only semi-implicit, with a single (or a limited number of) prediction and correction step(s), as in projection methods for incompressible flows (see [7,60] for seminal works and [19] for a review of most of the variants). The schemes which we propose in the present paper fall in this latter class.

We first deal with the barotropic Euler equations, which read:

$$
\begin{aligned}
& \partial_{t} \rho+\operatorname{div}(\rho \boldsymbol{u})=0, \\
& \partial_{t}(\rho \boldsymbol{u})+\operatorname{div}(\rho \boldsymbol{u} \otimes \boldsymbol{u})+\nabla p=0, \\
& \rho \geq 0, p=\wp(\rho)=\rho^{\gamma},
\end{aligned}
$$

where $t$ stands for the time, $\rho, \boldsymbol{u}, p$, are the density, velocity, pressure in the flow, and $\gamma>1$ is a coefficient specific to the considered fluid. For $p=\rho^{\gamma}, \gamma=2$ and $\rho=h$, we also get the shallow water (or Saint-Venant) equations.

Then, we address the full Euler equations, which are obtained from the complete Navier-Stokes equations

$$
\begin{aligned}
& \partial_{t} \rho+\operatorname{div}(\rho \boldsymbol{u})=0, \\
& \partial_{t}(\rho \boldsymbol{u})+\operatorname{div}(\rho \boldsymbol{u} \otimes \boldsymbol{u})+\nabla p-\operatorname{div}(\boldsymbol{\tau}(\boldsymbol{u}))=0, \\
& \partial_{t}(\rho E)+\operatorname{div}(\rho E \boldsymbol{u})+\operatorname{div}(p \boldsymbol{u})=0, \\
& p=(\gamma-1) \rho e, \quad E=\frac{1}{2}|\boldsymbol{u}|^{2}+e,
\end{aligned}
$$

by neglecting the viscous stress tensor, i.e. setting $\boldsymbol{\tau}(\boldsymbol{u})=0$. In this system, $E$ and $e$ stand for the total energy and the internal energy in the flow, respectively.

Both problems (1.1) and (1.2) are supposed to be posed over $\Omega \times(0, T)$, where $\Omega$ is an open bounded connected subset of $\mathbb{R}^{d}, 1 \leq d \leq 3$, and $(0, T)$ is a finite time interval. System (1.1) (resp. (1.2)) is complemented by initial conditions for $\rho$ and $\boldsymbol{u}$ (resp. $\rho, e$ and $\boldsymbol{u}$ ); these initial conditions are denoted by $\rho_{0}$ and $\boldsymbol{u}_{0}$ (resp. $\rho_{0}, e_{0}$ and $\boldsymbol{u}_{0}$ ), with $\rho_{0}>0$ and $e_{0}>0$. In both cases, we consider the boundary condition $\boldsymbol{u} \cdot \boldsymbol{n}=0$ at any time and a.e. on $\partial \Omega$, where $\boldsymbol{n}$ stands for the normal vector to the boundary.

This paper is organized as follows. We begin by describing the space discretizations (Sect. 2). We then address the barotropic case in Section 3; we introduce a fully implicit scheme and a pressure correction scheme (Sects. 3.1 and 3.2 resp.). We prove the stability of each algorithm and their consistency in the one-dimensional case, in the Lax-Wendroff sense. We finally propose in Section 4 a pressure correction scheme for the full Euler equations. We first give the general form of the algorithm (Sect. 4.2). The scheme solves the internal energy balance, which offers three advantages: first, we avoid the space discretization of the total energy, which involves cell-centered and face-centered variables; second, we obtain an algorithm which boils down to a usual pressure correction scheme in the incompressible limit; third, this relation implies that the internal energy remains positive. Consistency (again in the Lax-Wendroff sense) with the original total energy conservative equation is obtained thanks to corrective terms in the internal energy balance, designed to compensate numerical dissipation terms appearing in the discrete kinetic energy inequality. It is then shown in the 1D case (Sect. 4.3), that, supposing the convergence of a sequence of solutions, the limit is a weak solution of the continuous problem. Finally, we present some numerical tests in Section 4.4 for the case of the Euler equations (note that the numerical study of the correction pressure 
scheme in the barotropic case was performed in [27]). In several theoretical developments, we are lead to use a derived form of a discrete finite volume convection operator (for instance, typically, a convection operator for the kinetic energy, possibly with residual terms, obtained from the finite volume discretization of the convection of the velocity components); the proofs of various related discrete identities are given in the Appendix.

\section{MESHES AND UNKNOWNS}

Let $\mathcal{M}$ be a decomposition of the domain $\Omega$, supposed to be regular in the usual sense of the finite element literature (see e.g. [8]). The cells may be:

- for a general domain $\Omega$, either convex quadrilaterals $(d=2)$ or hexahedra $(d=3)$ or simplices, both types of cells being possibly combined in a same mesh,

- for a domain whose boundaries are hyperplanes normal to a coordinate axis, rectangles $(d=2)$ or rectangular parallelepipeds $(d=3)$ (the faces of which, of course, are then also necessarily normal to a coordinate axis).

By $\mathcal{E}$ and $\mathcal{E}(K)$ we denote the set of all $(d-1)$-faces $\sigma$ of the mesh and of the element $K \in \mathcal{M}$ respectively. The set of faces included in $\Omega$ (resp. in the boundary $\partial \Omega$ ) is denoted by $\mathcal{E}_{\text {int }}$ (resp. $\mathcal{E}_{\text {ext }}$ ); a face $\sigma \in \mathcal{E}_{\text {int }}$ separating the cells $K$ and $L$ is denoted by $\sigma=K \mid L$. The outward normal vector to a face $\sigma$ of $K$ is denoted by $\boldsymbol{n}_{K, \sigma}$. For $K \in \mathcal{M}$ and $\sigma \in \mathcal{E}$, we denote by $|K|$ the measure of $K$ and by $|\sigma|$ the $(d-1)$-measure of the face $\sigma$. For $1 \leq i \leq d$, we denote by $\mathcal{E}^{(i)} \subset \mathcal{E}$ and $\mathcal{E}_{\text {ext }}^{(i)} \subset \mathcal{E}_{\text {ext }}$ the subset of the faces of $\mathcal{E}$ and $\mathcal{E}_{\text {ext }}$ respectively which are perpendicular to the $i$ th unit vector of the canonical basis of $\mathbb{R}^{d}$.

The space discretization is staggered. In the case of rectangular or orthogonal parallelepipedic meshes, we use the Marker-And Cell (MAC) scheme [24,25]. For mixed simplicial or quadrilateral/hexahedral meshes, we use the discrete unknowns of the Crouzeix-Raviart [9] and Ranacher-Turek [58] finite element spaces; however, the associated finite element formulation is not used here (but it would readily provide a discretization of the diffusion terms, in the Navier-Stokes case $[1,16])$.

For all these space discretizations, the degrees of freedom for the scalar unknowns are associated with the cells of the mesh $\mathcal{M}$; these are the discrete pressure and density unknowns, and, for the full Euler equations, the internal energy unknowns, which are denoted by:

$$
\left\{p_{K}, \rho_{K}, e_{K}, K \in \mathcal{M}\right\} .
$$

Let us then turn to the degrees of freedom for the velocity (i.e. the discrete velocity unknowns).

- Rannacher-Turek or Crouzeix-Raviart discretizations - The discrete velocity unknowns are located at the center of the faces of the mesh, and represent the average of the velocity through the face. The set of discrete velocity unknowns reads:

$$
\left\{u_{\sigma, i}, \sigma \in \mathcal{E}, 1 \leq i \leq d\right\} .
$$

- MAC discretization - The degrees of freedom for the $i$ th component of the velocity are located at the center of the faces $\sigma \in \mathcal{E}^{(i)}$, so that the set of discrete velocity unknowns reads:

$$
\left\{u_{\sigma, i}, \sigma \in \mathcal{E}^{(i)}, 1 \leq i \leq d\right\} .
$$

We now introduce a dual mesh, which will be used for the finite volume approximation of the time derivative and convection terms in the momentum balance equation.

- Rannacher-Turek or Crouzeix-Raviart discretizations - For the RT or CR discretizations, the dual mesh is the same for all the velocity components. When $K \in \mathcal{M}$ is a simplex, a rectangle or a cuboid, for $\sigma \in \mathcal{E}(K)$, we define $D_{K, \sigma}$ as the cone with basis $\sigma$ and with vertex the mass center of $K$ (see Fig. 1). We thus obtain a partition of $K$ in $m$ sub-volumes, where $m$ is the number of faces of the mesh, each sub-volume 


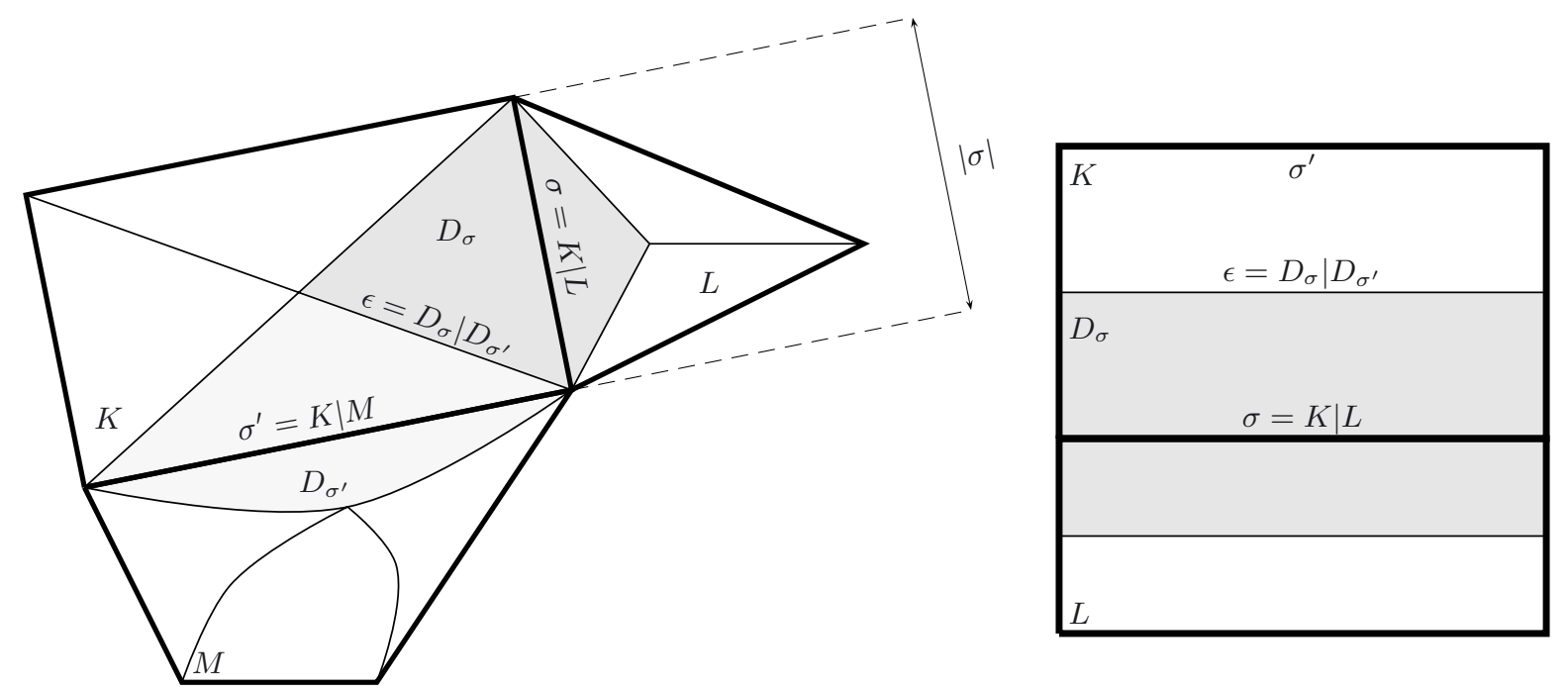

Figure 1. Notations for control volumes and dual cells - Left: Finite Elements (the present sketch illustrates the possibility, implemented in our software $\left(\mathrm{CALIF}^{3} \mathrm{~S}[5]\right)$, of mixing simplicial (Crouzeix-Raviart) and quadrangular (Rannacher-Turek) cells) - Right: MAC discretization, dual cell for the $y$-component of the velocity.

having the same measure $\left|D_{K, \sigma}\right|=|K| / m$. We extend this definition to general quadrangles and hexahedra, by supposing that we have built a partition still of equal-volume sub-cells, and with the same connectivities; note that this is of course always possible, but that such a volume $D_{K, \sigma}$ may be no longer a cone; indeed, if $K$ is far from a parallelogram, it may not be possible to build a cone having $\sigma$ as basis, the opposite vertex lying in $K$ and a volume equal to $|K| / m$. The volume $D_{K, \sigma}$ is referred to as the half-diamond cell associated with $K$ and $\sigma$.

For $\sigma \in \mathcal{E}_{\text {int }}, \sigma=K \mid L$, we now define the diamond cell $D_{\sigma}$ associated with $\sigma$ by $D_{\sigma}=D_{K, \sigma} \cup D_{L, \sigma}$; for an external face $\sigma \in \mathcal{E}_{\text {ext }} \cap \mathcal{E}(K), D_{\sigma}$ is just the same volume as $D_{K, \sigma}$.

- MAC discretization - For the MAC scheme, the dual mesh depends on the component of the velocity. For the $i$ th component, the dual cells are associated to the faces perpendicular to the $i$ th unit vector of the canonical basis of $\mathbb{R}^{d}$, i.e. to the faces of $\mathcal{E}^{(i)}$ (which is, of course, consistent with the location of the velocity discrete unknowns). A MAC dual cell only differs from the corresponding RT or CR one by the choice of the half-diamond cell, which, for $K \in \mathcal{M}$ and $\sigma \in \mathcal{E}(K)$, is now the rectangle or rectangular parallelepiped of basis $\sigma$ and of measure $\left|D_{K, \sigma}\right|=|K| / 2$.

We denote by $\left|D_{\sigma}\right|$ the measure of the dual cell $D_{\sigma}$, by $\epsilon=D_{\sigma} \mid D_{\sigma^{\prime}}$ the face separating two diamond cells $D_{\sigma}$ and $D_{\sigma^{\prime}}$, and by $\overline{\mathcal{E}}\left(D_{\sigma}\right)$ the set of the faces of $D_{\sigma}$.

Finally, we need to deal with the impermeability (i.e. $\boldsymbol{u} \cdot \boldsymbol{n}=0$ ) boundary condition. Since the velocity unknowns lie on the boundary (and not inside the cells), these conditions are taken into account in the definition of the discrete spaces. To avoid technicalities in the expression of the schemes, we suppose throughout this paper that the boundary is a.e. normal to a coordinate axis, (even in the case of the RT or CR discretizations), which allows to simply set to zero the corresponding velocity unknowns:

$$
\text { for } i=1, \ldots, d, \forall \sigma \in \mathcal{E}_{\text {ext }}^{(i)}, \quad u_{\sigma, i}=0 .
$$


Therefore, there are no discrete velocity unknowns on the boundary for the MAC scheme, and there are only $d-1$ discrete velocity unknowns on each boundary face for the CR and RT discretizations, which depend on the orientation of the face. In order to be able to write a unique expression of the discrete equations for both $\mathrm{MAC}$ and $\mathrm{CR} / \mathrm{RT}$ schemes, we introduce the set of faces $\mathcal{E}_{\mathcal{S}}^{(i)}$ associated with the degrees of freedom of each component of the velocity ( $\mathcal{S}$ stands for "scheme"):

$$
\mathcal{E}_{\mathcal{S}}^{(i)}=\mid \begin{aligned}
& \mathcal{E}^{(i)} \backslash \mathcal{E}_{\text {ext }}^{(i)} \text { for the MAC scheme, } \\
& \mathcal{E} \backslash \mathcal{E}_{\text {ext }}^{(i)} \text { for the CR or RT schemes. }
\end{aligned}
$$

Similarly, we unify the notation for the set of dual faces for both schemes by defining:

$$
\overline{\mathcal{E}}_{\mathcal{S}}^{(i)}=\mid \begin{aligned}
& \overline{\mathcal{E}}^{(i)} \backslash \overline{\mathcal{E}}_{\text {ext }}^{(i)} \text { for the MAC scheme, } \\
& \overline{\mathcal{E}} \backslash \overline{\mathcal{E}}_{\text {ext }}^{(i)} \text { for the CR or RT schemes, }
\end{aligned}
$$

where the symbol $\sim$ refers to the dual mesh; for instance, $\overline{\mathcal{E}}^{(i)}$ is thus the set of faces of the dual mesh associated with the $i$ th component of the velocity, and $\overline{\mathcal{E}}_{\text {ext }}^{(i)}$ stands for the subset of these dual faces included in the boundary. Note that, for the MAC scheme, the faces of $\overline{\mathcal{E}}^{(i)}$ are perpendicular to a unit vector of the canonical basis of $\mathbb{R}^{d}$, but not necessarily to the $i$ th one.

Note that general domains can easily be addressed (of course, with the CR or RT discretizations) by redefining, through linear combinations, the degrees of freedom at the external faces, so as to introduce the normal velocity as a new degree of freedom.

\section{IMPLiCIT AND SEMI-IMPLICIT SCHEMES FOR THE BAROTROPIC EQUATIONS}

We study two schemes for the numerical solution of System (1.1) which differ by the time discretization: the first one is implicit, and the second one is a non-iterative pressure-correction scheme introduced in [14]. This latter algorithm (and, by an easy extension, also the first one) was shown in [14] to have at least one solution, to provide solutions satisfying $\rho>0$ (and therefore also $p>0$ ) and to be unconditionally stable, in the sense that its (their) solution(s) satisfies an inequality corresponding to the control in $\mathrm{L}^{\infty}(0, T)$ of the integral of the discrete entropy over the domain. In this section, we complement this work in several directions. For the implicit scheme, we obtain the following results.

- First we pass from a (discrete) global (i.e. integrated over $\Omega$ ) entropy balance to (discrete) local balance equations. Precisely speaking, a discrete kinetic energy balance is established on dual cells, while a discrete potential elastic balance is established on primal cells.

These equations yield the stability of the scheme (i.e. the same global entropy conservation as in [14]) by a simple integration in space (i.e. summation over the primal and dual control volumes).

- Second, in one space dimension, the limit of any convergent sequence of solutions to the scheme is shown to be a weak solution to the continuous problem, and thus to satisfy the Rankine-Hugoniot conditions.

- Finally, passing to the limit in the discrete kinetic energy and elastic potential balances, such a limit is also shown to satisfy the usual entropy inequality.

For the pressure correction scheme, the results are essentially the same: the scheme is unconditionally stable, and the passage to the limit in the scheme shows that if a sequence of approximate solutions obtained with the scheme is assumed to converge to some limit, then the predicted and end-of-step velocities necessarily tend to the same function, and that the limit is a weak solution to the problem, satisfying the entropy inequality. The numerical study of this scheme (which is the only one implemented in practice) is performed in [27]. It confirms the present theoretical study: in particular, the scheme is observed to converge to weak entropy solutions of Riemann problems, with an approximately first order rate; in addition, it yields qualitatively correct solutions for CFL numbers much larger than one. 


\subsection{An implicit scheme}

\subsubsection{The scheme}

Let us consider a uniform partition $0=t_{0}<t_{1}<\ldots<t_{N}=T$ of the time interval (0,T), and let $\delta t=t_{n+1}-t_{n}$ for $n=0,1, \ldots, N-1$ be the constant time step. We consider an implicit-in-time scheme, which reads in its fully discrete form, for $0 \leq n \leq N-1$ :

$$
\forall K \in \mathcal{M}, \quad \frac{|K|}{\delta t}\left(\rho_{K}^{n+1}-\rho_{K}^{n}\right)+\sum_{\sigma \in \mathcal{E}(K)} F_{K, \sigma}^{n+1}=0,
$$

For $1 \leq i \leq d, \forall \sigma \in \mathcal{E}_{\mathcal{S}}^{(i)}$

$$
\begin{aligned}
& \quad \frac{\left|D_{\sigma}\right|}{\delta t}\left(\rho_{D_{\sigma}}^{n+1} u_{\sigma, i}^{n+1}-\rho_{D_{\sigma}}^{n} u_{\sigma, i}^{n}\right)+\sum_{\epsilon \in \overline{\mathcal{E}}\left(D_{\sigma}\right)} F_{\sigma, \epsilon}^{n+1} u_{\epsilon, i}^{n+1}-\left|D_{\sigma}\right|\left(\Delta^{\mathcal{M}} \boldsymbol{u}\right)_{\sigma, i}^{n+1}+\left|D_{\sigma}\right|(\nabla p)_{\sigma, i}^{n+1}=0, \\
& \forall K \in \mathcal{M}, \quad p_{K}^{n+1}=\wp\left(\rho_{K}^{n+1}\right)=\left(\rho_{K}^{n+1}\right)^{\gamma},
\end{aligned}
$$

where the terms introduced for each discrete equation are defined hereafter.

Equation (3.1a) is obtained by discretizing the mass balance (1.1a) over the primal mesh, and $F_{K, \sigma}^{n+1}$ stands for the mass flux across $\sigma$ outward $K$, which, because of the impermeability condition, vanishes on external faces and is given on the internal faces by:

$$
\forall \sigma=K\left|L \in \mathcal{E}_{\text {int }}, \quad F_{K, \sigma}^{n+1}=\right| \sigma \mid \rho_{\sigma}^{n+1} u_{K, \sigma}^{n+1},
$$

where $u_{K, \sigma}^{n+1}$ is an approximation of the normal velocity to the face $\sigma$ outward $K$. This latter quantity is defined by:

$$
u_{K, \sigma}^{n+1}=\mid \begin{aligned}
& u_{\sigma, i}^{n+1} \boldsymbol{e}^{(i)} \cdot \boldsymbol{n}_{K, \sigma} \text { for } \sigma \in \mathcal{E}^{(i)} \text { in the MAC case, } \\
& \boldsymbol{u}_{\sigma}^{n+1} \cdot \boldsymbol{n}_{K, \sigma} \text { in the CR and RT cases, }
\end{aligned}
$$

where $\boldsymbol{e}^{(i)}$ denotes the $i$ th vector of the orthonormal basis of $\mathbb{R}^{d}$. The density at the face $\sigma=K \mid L$ is approximated by the upwind technique:

$$
\rho_{\sigma}^{n+1}=\mid \begin{array}{ll}
\rho_{K}^{n+1} & \text { if } u_{K, \sigma}^{n+1} \geq 0 \\
\rho_{L}^{n+1} & \text { otherwise. }
\end{array}
$$

Note that the positivity of the density in (1.1c) is not enforced in the scheme but results from the above upwind choice (see e.g. [16], Lem. 2.1).

We now turn to the discrete momentum balance (3.1b). In order to obtain the desired estimates on the approximate solution such as a discrete kinetic energy inequality, the discretization of the momentum balance must be performed in a way that is compatible with the discretization of the mass balance equation. Hence we must carefully choose the values $\rho_{D_{\sigma}}^{n+1}$ and $\rho_{D_{\sigma}}^{n}$ as functions of the primal unknowns $\left(\rho_{K}^{n+1}\right)_{K \in \mathcal{M}}$ and $\left(\rho_{K}^{n}\right)_{K \in \mathcal{M}}$ and the fluxes on the dual faces $F_{\sigma, \epsilon}^{n+1}$ as functions of the fluxes on the primal faces $F_{K, \sigma}^{n+1}$. The values $\rho_{D_{\sigma}}^{n+1}$ and $\rho_{D_{\sigma}}^{n}$, which approximate the density on the face $\sigma$ at time $t^{n+1}$ and $t^{n}$ respectively, are given by the following weighted average:

$$
\text { for } \sigma=K \mid L \in \mathcal{E}_{\text {int }}, \text { for } k=n \text { and } k=n+1, \quad\left|D_{\sigma}\right| \rho_{D_{\sigma}}^{k}=\left|D_{K, \sigma}\right| \rho_{K}^{k}+\left|D_{L, \sigma}\right| \rho_{L}^{k} .
$$

Let us then detail the discretization of the convection term. The first task is to define the discrete mass flux through the dual face $\epsilon$ outward $D_{\sigma}$, denoted by $F_{\sigma, \epsilon}^{n+1}$; the guideline for its construction is that a finite volume discretization of the mass balance equation over the diamond cells, of the form

$$
\forall \sigma \in \mathcal{E}, \quad \frac{\left|D_{\sigma}\right|}{\delta t}\left(\rho_{D_{\sigma}}^{n+1}-\rho_{D_{\sigma}}^{n}\right)+\sum_{\epsilon \in \mathcal{E}\left(D_{\sigma}\right)} F_{\sigma, \epsilon}^{n+1}=0
$$


must hold in order to be able to derive a discrete kinetic energy balance (see Sect. 3.1.1). For the MAC scheme, the flux on a dual face which is located on two primal faces is the mean value of the sum of the fluxes on these two primal faces, and the flux of a dual face located between two primal faces is again the mean value of the sum of the fluxes on these two primal faces [28]. In the case of the CR and RT schemes, for a dual face $\epsilon$ included in the primal cell $K$, this flux is computed as a linear combination (with constant coefficients, i.e. independent of the cell) of the mass fluxes through the faces of $K$, i.e. the quantities $\left(F_{K, \sigma}^{n+1}\right)_{\sigma \in \mathcal{E}(K)}$ appearing in the discrete mass balance (3.1a). We refer to [1,15] for a detailed construction of this approximation. Let us remark that a dual face lying on the boundary is then also a primal face, and the flux across that face is zero. Therefore, the values $u_{\epsilon, i}^{n+1}$ are only needed at the internal dual faces; we choose them to be centered (in fact, the upwind choice is also covered by our analysis, see the comments on the numerical diffusion below); so, for $\epsilon=D_{\sigma} \mid D_{\sigma^{\prime}}$, $u_{\epsilon, i}^{n+1}$ reads:

$$
u_{\epsilon, i}^{n+1}=\frac{u_{\sigma, i}^{n+1}+u_{\sigma^{\prime}, i}^{n+1}}{2}
$$

The quantity $\left(\Delta^{\mathcal{M}} \boldsymbol{u}\right)_{\sigma, i}^{n+1}$ stands for a possible stabilizing diffusion term which may be written under a finite volume form over any diamond cell $D_{\sigma}$ associated with the $i$ th component of the velocity:

$$
-\left|D_{\sigma}\right|\left(\Delta^{\mathcal{M}} \boldsymbol{u}\right)_{\sigma, i}^{n+1}=\sum_{\epsilon=D_{\sigma} \mid D_{\sigma^{\prime}}} \nu h_{\epsilon}^{d-2}\left(u_{\sigma, i}^{n+1}-u_{\sigma^{\prime}, i}^{n+1}\right),
$$

where $h_{\epsilon}$ is a characteristic dimension of the face $\epsilon$, and $\nu$ stands for a non-negative coefficient, possibly depending on a power of $h_{\epsilon}$. Note that external faces are excluded in the sum at the right-hand side, which means that the possible associated diffusion flux is set to zero. Note also that $\left(\Delta^{\mathcal{M}} \boldsymbol{u}\right)_{\sigma, i}^{n+1}$ is usually (i.e. for general meshes) not consistent with a Laplace operator. Moreover, the upwind scheme

$$
u_{\epsilon, i}^{n+1}=\mid \begin{array}{ll}
u_{\sigma, i}^{n+1} & \text { if } F_{\sigma, \epsilon}^{n+1} \geq 0, \\
u_{\sigma^{\prime}, i}^{n+1} & \text { otherwise, }
\end{array}
$$

can also be written under this form, since in this case, the convection term may be written as:

$$
\left(F_{\sigma, \epsilon}^{n+1} u_{\epsilon, i}^{n+1}\right)^{(\mathrm{up})}=F_{\sigma, \epsilon}^{n+1} \frac{u_{\sigma, i}^{n+1}+u_{\sigma^{\prime}, i}^{n+1}}{2}+\frac{1}{2}\left|F_{\sigma, \epsilon}^{n+1}\right|\left(u_{\sigma, i}^{n+1}-u_{\sigma^{\prime}, i}^{n+1}\right) \text { for } \epsilon=D_{\sigma} \mid D_{\sigma^{\prime}} .
$$

Hence the upwind choice is included in the formulation (3.1b), (3.6), with a numerical diffusion term defined by (3.7), setting

$$
\nu h_{\epsilon}^{d-2}=\frac{1}{2}\left|F_{\sigma, \epsilon}^{n+1}\right|,
$$

and $\nu$ behaves as $h_{\epsilon}$ in this case (provided that the density and the velocity are uniformly bounded).

The introduction of a numerical diffusion of the form (3.7) presents two advantages:

- On one hand, if we assume that the coefficient $\nu$ is such that $C_{1} h_{\epsilon}^{\alpha} \leq \nu \leq C_{2} h_{\epsilon}^{\alpha}$ with $C_{1}, C_{2} \in \mathbb{R}_{+}$and $0<\alpha<2$, we obtain a weak $\mathrm{L}^{2}\left(\mathrm{H}^{1}\right)$ control of the velocity which is sufficient, at least in one space dimension, to pass to the limit in the scheme (see Sect. 3.1.3). This is not the the case for a pure (i.e. without additional diffusion term) upwind discretization, because $\nu$ vanishes with the mass flux (i.e. the normal velocity).

Note however that the situation is different if we now assume that the approximate velocity $\boldsymbol{u}$ satisfies a $\mathrm{B} V$ estimate: the convergence analysis of Section 3.1.3 then still holds in the centered or upwind case, without requiring any additional diffusion.

- On the other hand, this formalism may prepare for a stabilization strategy which could be less diffusive than the upwind choice, choosing for instance $\nu$ on the basis of an a posteriori analysis of the local regularity of the solution $[20,21,40]$. 
The last term $(\nabla p)_{\sigma, i}^{n+1}$ stands for the $i$ th component of the discrete pressure gradient at the face $\sigma$. The gradient operator is built as the transpose of the natural discrete divergence operator which is defined by

$$
|K|(\operatorname{div} \boldsymbol{u})_{K}=\sum_{\sigma \in \mathcal{E}(K)}|\sigma| u_{K, \sigma}
$$

In the CR and RT case, the duality between the divergence and gradient operators simply reads:

$$
\sum_{K \in \mathcal{M}}|K| p_{K}(\operatorname{div} \boldsymbol{u})_{K}+\sum_{\sigma \in \mathcal{E}}\left|D_{\sigma}\right| \boldsymbol{u}_{\sigma} \cdot(\nabla p)_{\sigma}=0
$$

This duality relation may be rewritten so as to fit both the CR/RT scheme and the MAC scheme as follows:

$$
\sum_{K \in \mathcal{M}}|K| p_{K}(\operatorname{div} \boldsymbol{u})_{K}+\sum_{i=1}^{d} \sum_{\sigma \in \mathcal{E}_{\mathcal{S}}^{(i)}}\left|D_{\sigma}\right| u_{\sigma, i}(\nabla p)_{\sigma, i}=0 .
$$

Therefore, on any internal face, the components of the gradients are given by:

$$
\text { for } \sigma=K \mid L \in \mathcal{E}_{\text {int }}, \quad(\nabla p)_{\sigma, i}^{n+1}=\frac{|\sigma|}{\left|D_{\sigma}\right|}\left(p_{L}^{n+1}-p_{K}^{n+1}\right) \boldsymbol{n}_{K, \sigma} \cdot \boldsymbol{e}^{(i)} .
$$

Note that, because of the impermeability boundary conditions, the discrete gradient is not defined at the external faces.

Finally, the initial approximations for $\rho$ and $\boldsymbol{u}$ are given by the average of the initial conditions $\rho_{0}$ and $\boldsymbol{u}_{0}$ on the primal and dual cells respectively:

$$
\begin{aligned}
& \forall K \in \mathcal{M}, \quad \rho_{K}^{0}=\frac{1}{|K|} \int_{K} \rho_{0}(\boldsymbol{x}) \mathrm{d} \boldsymbol{x} \\
& \text { for } 1 \leq i \leq d, \forall \sigma \in \mathcal{E}_{\mathcal{S}}^{(i)}, \quad u_{\sigma, i}^{0}=\frac{1}{\left|D_{\sigma}\right|} \int_{D_{\sigma}}\left(\boldsymbol{u}_{0}(\boldsymbol{x})\right)_{i} \mathrm{~d} \boldsymbol{x} .
\end{aligned}
$$

\subsubsection{Estimates}

We begin with an estimate on the velocity which is a discrete equivalent of the kinetic energy balance. Recall that in the continuous setting, the kinetic energy balance is formally obtained by multiplying the $i$ th component of the momentum balance equation (1.1b) by the $i$ th component $u_{i}$ of $\boldsymbol{u}$; this yields for $1 \leq i \leq d$, using the mass balance equation (1.1a) twice:

$$
\partial_{t}\left(\frac{1}{2} \rho u_{i}^{2}\right)+\operatorname{div}\left(\left(\frac{1}{2} \rho u_{i}^{2}\right) \boldsymbol{u}\right)+\left(\partial_{x_{i}} p\right) u_{i}=0
$$

and thus, summing over the components:

$$
\partial_{t}\left(\rho E_{k}\right)+\operatorname{div}\left(\rho E_{k} \boldsymbol{u}\right)+\nabla p \cdot \boldsymbol{u}=0, \quad \text { with } E_{k}=\frac{1}{2}|\boldsymbol{u}|^{2} .
$$

In the discrete setting, this multiplication must be performed on the dual mesh, since the velocity unknowns are defined on the faces. This is the reason why we chose the fluxes on the faces of the dual mesh in such a way that a discrete mass balance equation holds on the dual grid cells, thus allowing us to use Lemma A.2 (which performs the discrete equivalent of the above formal computations) on the dual mesh. 
Lemma 3.1 (Discrete kinetic energy balance, implicit scheme). A solution to the system (3.1) satisfies the following equality, for $1 \leq i \leq d, \sigma \in \mathcal{E}_{\mathcal{S}}^{(i)}$ and $0 \leq n \leq N-1$ :

$$
\frac{1}{2} \frac{\left|D_{\sigma}\right|}{\delta t}\left[\rho_{D_{\sigma}}^{n+1}\left(u_{\sigma, i}^{n+1}\right)^{2}-\rho_{D_{\sigma}}^{n}\left(u_{\sigma, i}^{n}\right)^{2}\right]+\frac{1}{2} \sum_{\epsilon=D_{\sigma} \mid D_{\sigma^{\prime}}} F_{\sigma, \epsilon}^{n+1} u_{\sigma, i}^{n+1} u_{\sigma^{\prime}, i}^{n+1}+\left|D_{\sigma}\right|(\nabla p)_{\sigma, i}^{n+1} u_{\sigma, i}^{n+1}=-R_{\sigma, i}^{n+1}
$$

where

$$
R_{\sigma, i}^{n+1}=\frac{\left|D_{\sigma}\right|}{2 \delta t} \rho_{D_{\sigma}}^{n}\left(u_{\sigma, i}^{n+1}-u_{\sigma, i}^{n}\right)^{2}+\left[\sum_{\epsilon=D_{\sigma} \mid D_{\sigma^{\prime}}} \nu h_{\epsilon}^{d-2}\left(u_{\sigma, i}^{n+1}-u_{\sigma^{\prime}, i}^{n+1}\right)\right] u_{\sigma, i}^{n+1} .
$$

Proof. Let us multiply equation (3.1b) by the corresponding velocity unknown $u_{\sigma, i}^{n+1}$; this yields

$$
T_{\sigma, i}^{\mathrm{conv}}+T_{\sigma, i}^{\Delta}+T_{\sigma, i}^{\nabla}=0
$$

with:

$$
\begin{aligned}
& T_{\sigma, i}^{\mathrm{conv}}=\left[\frac{\left|D_{\sigma}\right|}{\delta t}\left(\rho_{D_{\sigma}}^{n+1} u_{\sigma, i}^{n+1}-\rho_{D_{\sigma}}^{n} u_{\sigma, i}^{n}\right)+\sum_{\epsilon=D_{\sigma} \mid D_{\sigma^{\prime}}} F_{\sigma, \epsilon}^{n+1} u_{\epsilon, i}^{n+1}\right] u_{\sigma, i}^{n+1}, \\
& T_{\sigma, i}^{\Delta}=\left[\sum_{\epsilon=D_{\sigma} \mid D_{\sigma^{\prime}}} \nu h_{\epsilon}^{d-2}\left(u_{\sigma, i}^{n+1}-u_{\sigma^{\prime}, i}^{n+1}\right)\right] u_{\sigma, i}^{n+1}, \\
& T_{\sigma, i}^{\nabla}=\left|D_{\sigma}\right|(\nabla p)_{\sigma, i}^{n+1} u_{\sigma, i}^{n+1} .
\end{aligned}
$$

Applying Lemma A.2 with $P=D_{\sigma}$, we get from the identity (A.9) that

$$
T_{\sigma, i}^{\mathrm{conv}}=\frac{1}{2} \frac{\left|D_{\sigma}\right|}{\delta t}\left[\rho_{D_{\sigma}}^{n+1}\left(u_{\sigma, i}^{n+1}\right)^{2}-\rho_{D_{\sigma}}^{n}\left(u_{\sigma, i}^{n}\right)^{2}\right]+\frac{1}{2} \sum_{\epsilon=D_{\sigma} \mid D_{\sigma^{\prime}}} F_{\sigma, \epsilon}^{n+1} u_{\sigma, i}^{n+1} u_{\sigma^{\prime}, i}^{n+1}+\frac{\left|D_{\sigma}\right|}{2 \delta t} \rho_{D_{\sigma}}^{n}\left(u_{\sigma, i}^{n+1}-u_{\sigma, i}^{n}\right)^{2} .
$$

We then note that

$$
T_{\sigma, i}^{\Delta}+\frac{\left|D_{\sigma}\right|}{2 \delta t} \rho_{D_{\sigma}}^{n}\left(u_{\sigma, i}^{n+1}-u_{\sigma, i}^{n}\right)^{2}=R_{\sigma, i}^{n+1}
$$

where $R_{\sigma, i}^{n+1}$ is defined by (3.15), which concludes the proof of (3.14).

Let us now define the elastic potential $\mathcal{P}$ :

$$
\mathcal{P}(z)=\int_{0}^{z} \frac{\wp(s)}{s^{2}} \text { ds i.e. } \mathcal{P}(z)=\left\{\begin{array}{llc}
\frac{z^{\gamma-1}}{\gamma-1} & \text { if } & \gamma>1 \\
\ln (z) & \text { if } & \gamma=1
\end{array}\right.
$$

and let $\mathcal{H}$ be the function defined over $(0,+\infty)$ by

$$
\mathcal{H}(z)=z \mathcal{P}(z)=\left\{\begin{array}{lll}
\frac{z^{\gamma}}{\gamma-1} & \text { if } & \gamma>1 \\
z \ln (z) & \text { if } & \gamma=1
\end{array}\right.
$$

It may easily be checked that $z \mathcal{H}^{\prime}(z)-\mathcal{H}(z)=\wp(z)$; therefore, by a formal computation detailed in the appendix (see (A.3)), multiplying (1.1a) by $\mathcal{H}^{\prime}(\rho)$ yields:

$$
\partial_{t}(\mathcal{H}(\rho))+\operatorname{div}(\mathcal{H}(\rho) \boldsymbol{u})+p \operatorname{div}(\boldsymbol{u})=0 .
$$

We now derive a discrete analogue of this relation. 
Lemma 3.2 (Discrete potential balance). Let $\mathcal{H}$ be defined by (3.17). A solution to the system (3.1) satisfies the following equality, for $K \in \mathcal{M}$ and $0 \leq n \leq N-1$ :

$$
\frac{|K|}{\delta t}\left[\mathcal{H}\left(\rho_{K}^{n+1}\right)-\mathcal{H}\left(\rho_{K}^{n}\right)\right]+\sum_{\sigma \in \mathcal{E}(K)}|\sigma| \mathcal{H}\left(\rho_{\sigma}^{n+1}\right) u_{K, \sigma}^{n+1}+|K| p_{K}^{n+1}\left(\operatorname{div} \boldsymbol{u}^{n+1}\right)_{K}=-R_{K}^{n+1},
$$

with:

$$
R_{K}^{n+1}=\frac{|K|}{2 \delta t} \mathcal{H}^{\prime \prime}\left(\bar{\rho}_{K}^{n+\frac{1}{2}}\right)\left(\rho_{K}^{n+1}-\rho_{K}^{n}\right)^{2}+\frac{1}{2} \sum_{\sigma=K \mid L}|\sigma|\left(u_{K, \sigma}^{n+1}\right)^{-} \mathcal{H}^{\prime \prime}\left(\bar{\rho}_{\sigma}^{n+1}\right)\left(\rho_{\sigma}^{n+1}-\rho_{K}^{n+1}\right)^{2},
$$

where $\bar{\rho}_{K}^{n+\frac{1}{2}} \in\left[\min \left(\rho_{K}^{n+1}, \rho_{K}^{n}\right), \max \left(\rho_{K}^{n+1}, \rho_{K}^{n}\right)\right], \bar{\rho}_{\sigma}^{n+1} \in\left[\min \left(\rho_{\sigma}^{n+1}, \rho_{K}^{n+1}\right), \max \left(\rho_{\sigma}^{n+1}, \rho_{K}^{n+1}\right)\right]$ for all $\sigma \in \mathcal{E}(K)$, and, for $a \in \mathbb{R}, a^{-} \geq 0$ is defined by $a^{-}=-\min (a, 0)$. Note that, since the function $\mathcal{H}$ is convex, $R_{K}^{n+1}$ is non-negative.

Proof. Let us multiply the discrete mass balance (3.1a) by $\mathcal{H}^{\prime}\left(\rho_{K}^{n+1}\right)$. The result is then a consequence of Lemma A.1 with $P=K$, using the fact that $z \mathcal{H}^{\prime}(z)-\mathcal{H}(z)=\wp(z)$ and that $\rho_{\sigma}^{n+1}$ is the upwind choice between $\rho_{K}$ and $\rho_{L}$ in the remainder term $R_{K, \delta t}$.

Summing (3.13) and (3.18) yields:

$$
\partial_{t} \eta+\operatorname{div}((\eta+p) \boldsymbol{u})=0,
$$

where $\eta=\rho E_{k}+\mathcal{H}(\rho)$ is the entropy of the system. This relation is only valid for regular solutions, and should be replaced by an inequality to take into account the presence of shocks (see relations (3.34)-(3.35)). Integrating over $\Omega$ and using the boundary conditions yields:

$$
\frac{\mathrm{d}}{\mathrm{d} t} \int_{\Omega} \eta(\boldsymbol{x}, t) \mathrm{d} \boldsymbol{x} \leq 0 \quad\left(\text { for regular solutions, } \frac{\mathrm{d}}{\mathrm{d} t} \int_{\Omega} \eta(\boldsymbol{x}, t) \mathrm{d} \boldsymbol{x}=0\right),
$$

and, for $t \in(0, T)$,

$$
\int_{\Omega} \eta(\boldsymbol{x}, t) \mathrm{d} \boldsymbol{x} \leq \int_{\Omega} \eta(\boldsymbol{x}, 0) \mathrm{d} \boldsymbol{x} .
$$

The following proposition states a discrete analogue to this relation.

Proposition 3.3 (Global discrete entropy inequality, existence of a solution). Assume that the initial density $\rho_{0}$ is positive. Then, there exists a solution $\left(\boldsymbol{u}^{n}, \rho^{n}\right)_{0 \leq n \leq N}$ to the scheme (3.1), and, for $1 \leq n \leq N, \rho^{n}>0$ and the following inequality holds:

$$
\frac{1}{2} \sum_{i=1}^{d} \sum_{\sigma \in \mathcal{E}_{\mathcal{S}}^{(i)}}\left|D_{\sigma}\right| \rho_{D_{\sigma}}^{n}\left(u_{\sigma, i}^{n}\right)^{2}+\sum_{K \in \mathcal{M}}|K| \mathcal{H}\left(\rho_{K}^{n}\right)+\mathcal{R}^{n} \leq C_{0},
$$

where $C_{0} \in \mathbb{R}_{+}$only depends on the initial conditions, and $\mathcal{R}^{n}$ is the following non-negative remainder which depends on the space and time translates of the unknowns:

$$
\begin{aligned}
\mathcal{R}^{n}= & \sum_{i=1}^{d} \sum_{k=1}^{n}\left[\frac{1}{2} \sum_{\sigma \in \mathcal{E}_{\mathcal{S}}^{(i)}}\left|D_{\sigma}\right| \rho_{D_{\sigma}}^{k-1}\left(u_{\sigma, i}^{k}-u_{\sigma, i}^{k-1}\right)^{2}+\delta t \sum_{\epsilon=D_{\sigma} \mid D_{\sigma^{\prime}} \in \overline{\mathcal{E}}_{\mathcal{S}}^{(i)}} \nu h_{\sigma}^{d-2}\left(u_{\sigma, i}^{k}-u_{\sigma^{\prime}, i}^{k}\right)^{2}\right] \\
& +\frac{\gamma}{2} \sum_{k=1}^{n} \delta t \sum_{\sigma=K \mid L \in \mathcal{E}_{\mathrm{int}}}|\sigma|\left(\rho_{\sigma, \gamma}^{k}\right)^{\gamma-2}\left|u_{K, \sigma}^{k}\right|\left(\rho_{K}^{k}-\rho_{L}^{k}\right)^{2},
\end{aligned}
$$

with $\rho_{\sigma, \gamma}^{k}$ equal to either $\rho_{K}^{k}$ or $\rho_{L}^{k}$ and such that $\left(\rho_{\sigma, \gamma}^{k}\right)^{\gamma-2}=\min \left(\left(\rho_{K}^{k}\right)^{\gamma-2},\left(\rho_{L}^{k}\right)^{\gamma-2}\right)$. 
Remark 3.4. For $\gamma>1$, the function $\mathcal{H}$ is positive and increasing over $(0,+\infty)$. The inequality (3.21) thus readily provides an estimate on the unknowns.

This is still true also for $\gamma=1$, since in this case $\mathcal{H}(z)=z \ln z$ and therefore $\mathcal{H}(z) \geq-1 / e, \forall z \in(0,+\infty)$, and $\mathcal{H}$ is increasing over $(1 / e,+\infty)$. In fact, in order to get the usual formulation of an estimate, we may rephrase the inequality (3.21) by changing the expression of $\mathcal{H}$ to $\mathcal{H}(z)=\max (z \log (z), 0)$ and adding $|\Omega| / e$ to the constant $C$ at the right-hand side.

Proof. Let us give the proof of Proposition 3.3. The positivity of the density is a consequence of the properties of the upwind choice (3.3) for $\rho$ [16], Lemma 2.1; note that it may also be proved applying Lemma A.1 with $\psi(s)=\frac{1}{2}\left(s^{-}\right)^{2}$ and $P=K$.

Let us then sum equation (3.14) over the components $i$ and the faces $\sigma \in \mathcal{E}_{\mathcal{S}}^{(i)}$, equation (3.19) over $K \in \mathcal{M}$, and, finally, the two obtained relations. Since the discrete gradient and divergence operators are dual with respect to the $\mathrm{L}^{2}$-inner product (see (3.11)), noting that the conservative fluxes vanish in the summation, we get, for $1 \leq k \leq N$ :

$\frac{1}{2} \sum_{i=1}^{d} \sum_{\sigma \in \mathcal{E}_{\mathcal{S}}^{(i)}} \frac{\left|D_{\sigma}\right|}{\delta t}\left[\rho_{D_{\sigma}}^{k}\left(u_{\sigma, i}^{k}\right)^{2}-\rho_{D_{\sigma}}^{k-1}\left(u_{\sigma, i}^{k-1}\right)^{2}\right]+\sum_{K \in \mathcal{M}} \frac{|K|}{\delta t}\left(\mathcal{H}\left(\rho_{K}^{k}\right)-\mathcal{H}\left(\rho_{K}^{k-1}\right)\right)=-\sum_{i=1}^{d} \sum_{\sigma \in \mathcal{E}_{\mathcal{S}}^{(i)}} R_{\sigma, i}^{k}-\sum_{K \in \mathcal{M}} R_{K}^{k}$.

Summing (3.23) for $k=1$ to $n$, and using the fact that $\mathcal{H}^{\prime \prime}(z)=\gamma z^{\gamma-2}$ for any $\gamma \geq 1$ yields (3.21), with $\mathcal{R}^{n}$ given by (3.22) and

$$
C_{0}=\frac{1}{2} \sum_{i=1}^{d} \sum_{\sigma \in \mathcal{E}_{\mathcal{S}}^{(i)}}\left|D_{\sigma}\right| \rho_{\sigma}^{0}\left|u_{\sigma, i}^{0}\right|^{2}+\sum_{K \in \mathcal{M}}|K| \mathcal{H}\left(\rho_{K}^{0}\right) .
$$

Finally, the existence of a solution may be inferred by the Brouwer fixed point theorem, by an easy adaptation of the proof of ([12], Prop. 5.2). This proof relies on the following set of mesh-dependent estimates: the conservativity of the mass balance discretization, together with the fact that the density is positive, yields an estimate for $\rho$ in the $\mathrm{L}^{1}$-norm, and so, by a norm equivalence argument, of the pressure in any norm; the discrete momentum balance equation then provides a control on the velocity. Therefore, computing

(i) $\rho$ from the mass balance for fixed $\boldsymbol{u}$;

(ii) $p$ from $\rho$ by the equation of state;

(iii) and finally $\boldsymbol{u}$ from the momentum balance equation with fixed $\rho$ and $p$.

yields an iteration in a bounded convex subset of a finite dimensional space.

\subsubsection{Passing to the limit in the scheme}

The objective of this section is to show, in the one dimensional case, that if a sequence of solutions is controlled in suitable norms and converges to a limit, this latter necessarily satisfies a (part of the) weak formulation of the continuous problem.

The 1D version of the scheme which is studied in this section may be obtained from Scheme (3.1) by taking the MAC variant, with only one horizontal stripe of grid cells, supposing that the vertical component of the velocity (the degrees of freedom of which are located on the top and bottom boundaries) vanishes, and that the measure of the vertical faces is equal to 1 . For the sake of readability, however, we completely rewrite this 1D scheme, and, to this purpose, we first introduce some adaptations of the notations to the one dimensional case. For any $K \in \mathcal{M}$, we denote by $h_{K}$ its length (so $h_{K}=|K|$ ); when we write $K=\left[\sigma \sigma^{\prime}\right]$, this means that either $K=\left(x_{\sigma}, x_{\sigma^{\prime}}\right)$ or $K=\left(x_{\sigma^{\prime}}, x_{\sigma}\right)$; if we need to specify the order, i.e. $K=\left(x_{\sigma}, x_{\sigma^{\prime}}\right)$ with $x_{\sigma}<x_{\sigma^{\prime}}$, then we write $K=\left[\overrightarrow{\sigma \sigma^{\prime}}\right]$. For an interface $\sigma=K \mid L$ between two cells $K$ and $L$, we define $h_{\sigma}=\left(h_{K}+h_{L}\right) / 2$, so, by definition of the dual mesh, $h_{\sigma}=\left|D_{\sigma}\right|$. If we need to specify the order of the cells $K$ and $L$, say $K$ is left of $L$, 
then we write $\sigma=\overrightarrow{K \mid L}$. With these notations, the implicit scheme (3.1) may be written as follows in the one dimensional setting:

$$
\begin{aligned}
& \forall K \in \mathcal{M}, \quad \rho_{K}^{0}=\frac{1}{|K|} \int_{K} \rho_{0}(x) \mathrm{d} x \\
& \forall \sigma \in \mathcal{E}_{\text {int }}, \quad u_{\sigma}^{0}=\frac{1}{\left|D_{\sigma}\right|} \int_{D_{\sigma}} u_{0}(x) \mathrm{d} x, \\
& \forall K=\left[\overrightarrow{\sigma \sigma^{\prime}}\right] \in \mathcal{M}, \\
& \qquad \frac{|K|}{\delta t}\left(\rho_{K}^{n+1}-\rho_{K}^{n}\right)+F_{\sigma^{\prime}}^{n+1}-F_{\sigma}^{n+1}=0, \\
& \forall \sigma=\overrightarrow{K \mid L} \in \mathcal{E}_{\text {int }}, \quad \frac{\left|D_{\sigma}\right|}{\delta t}\left(\rho_{D_{\sigma}}^{n+1} u_{\sigma}^{n+1}-\rho_{D_{\sigma}}^{n} u_{\sigma}^{n}\right)+F_{L}^{n+1} u_{L}^{n+1}-F_{K}^{n+1} u_{K}^{n+1} \\
& \forall K \in \mathcal{M}, \quad p_{K}^{n+1}=\wp\left(\rho_{K}^{n+1}\right)=\left(\rho_{K}^{n+1}\right)^{\gamma} .
\end{aligned}
$$

The mass flux in the discrete mass balance equation is given, for $\sigma \in \mathcal{E}_{\text {int }}$, by:

$$
F_{\sigma}^{n+1}=\rho_{\sigma}^{n+1} u_{\sigma}^{n+1},
$$

where the upwind approximate density $\rho_{\sigma}^{n+1}$ at the face $\sigma$ is defined by (3.3). In the momentum balance equation, the application of the procedure described in Section 3.1.1 yields the following expression for the density associated with the dual cell $D_{\sigma}$ with $\sigma=K \mid L$ and for the mass fluxes at the dual face located at the center of the cell $K=\left[\sigma \sigma^{\prime}\right]$ :

$$
\rho_{D_{\sigma}}^{n+1}=\frac{1}{2\left|D_{\sigma}\right|}\left(|K| \rho_{K}^{n+1}+|L| \rho_{L}^{n+1}\right), \quad F_{K}^{n+1}=\frac{1}{2}\left(F_{\sigma}^{n+1}+F_{\sigma^{\prime}}^{n+1}\right),
$$

and the approximation of the velocity at this face is centered: $u_{K}^{n+1}=\left(u_{\sigma}^{n+1}+u_{\sigma^{\prime}}^{n+1}\right) / 2$. Finally, for a face $\sigma=\overrightarrow{K \mid L}$ with $K=\left[\overrightarrow{\sigma^{\prime} \sigma}\right]$ and $L=\left[\overrightarrow{\sigma \sigma^{\prime \prime}}\right]$, the stabilization diffusion term reads:

$$
-\left|D_{\sigma}\right|\left(\Delta_{\mathcal{M}} u\right)_{\sigma}^{n+1}=\nu\left[\frac{1}{h_{K}}\left(u_{\sigma}^{n+1}-u_{\sigma^{\prime}}^{n+1}\right)+\frac{1}{h_{L}}\left(u_{\sigma}^{n+1}-u_{\sigma^{\prime \prime}}^{n+1}\right)\right] .
$$

Definition 3.5 (Regular sequence of discretizations). We define a regular sequence of discretizations $\left(\mathcal{M}^{(m)}, \delta t^{(m)}, \nu^{(m)}\right)_{m \in \mathbb{N}}$ as a sequence of meshes, time steps and numerical diffusion coefficients satisfying the following assertions:

(i) both the time step $\delta t^{(m)}$ and the size $h^{(m)}$ of the mesh $\mathcal{M}^{(m)}$, defined by $h^{(m)}=\sup _{K \in \mathcal{M}^{(m)}} h_{K}$, tend to zero as $m \rightarrow+\infty$;

(ii) there exists $\theta>0$ such that:

$$
\theta \leq \frac{h_{K}}{h_{L}} \leq \frac{1}{\theta}, \quad \forall m \in \mathbb{N} \text { and } K, L \in \mathcal{M}^{(m)} \text { sharing a face, }
$$

(iii) the sequence of numerical diffusion coefficients $\left(\nu^{(m)}\right)_{m \in \mathbb{N}}$ is such that:

$$
\lim _{m \rightarrow+\infty} \nu^{(m)}=0, \quad \lim _{m \rightarrow+\infty} \frac{\left(h^{(m)}\right)^{2}}{\nu^{(m)}}=0 .
$$


Let such a regular sequence of discretizations be given, and $\rho^{(m)}, p^{(m)}$ and $u^{(m)}$ be a solution given by the scheme (3.24) with the mesh $\mathcal{M}^{(m)}$, the time step $\delta t^{(m)}$ and the numerical diffusion coefficient $\nu^{(m)}$. To the discrete unknowns, we associate piecewise constant functions on time intervals and on primal or dual meshes, so that the density $\rho^{(m)}$, the pressure $p^{(m)}$ and the velocity $u^{(m)}$ are defined almost everywhere on $\Omega \times(0, T)$ by:

$$
\begin{aligned}
\rho^{(m)}(x, t) & =\sum_{n=0}^{N-1} \sum_{K \in \mathcal{M}}\left(\rho^{(m)}\right)_{K}^{n+1} \mathcal{X}_{K}(x) \mathcal{X}_{(n, n+1]}(t), \quad p^{(m)}(x, t)=\sum_{n=0}^{N-1} \sum_{K \in \mathcal{M}}\left(p^{(m)}\right)_{K}^{n+1} \mathcal{X}_{K}(x) \mathcal{X}_{(n, n+1]}(t), \\
u^{(m)}(x, t) & =\sum_{n=0}^{N-1} \sum_{\sigma \in \mathcal{E}}\left(u^{(m)}\right)_{\sigma}^{n+1} \mathcal{X}_{D_{\sigma}}(x) \mathcal{X}_{(n, n+1]}(t),
\end{aligned}
$$

where $\mathcal{X}_{K}, \mathcal{X}_{D_{\sigma}}$ and $\mathcal{X}_{(n, n+1]}$ stand for the characteristic functions of the intervals $K, D_{\sigma}$ and $\left(t^{n}, t^{n+1}\right]$ respectively.

A weak solution to the continuous problem satisfies, for any $\varphi \in \mathrm{C}_{c}^{\infty}(\Omega \times[0, T))$ :

$$
\begin{aligned}
& -\int_{0}^{T} \int_{\Omega}\left[\rho \partial_{t} \varphi+\rho u \partial_{x} \varphi\right] \mathrm{d} x \mathrm{~d} t-\int_{\Omega} \rho_{0}(x) \varphi(x, 0) \mathrm{d} x=0 \\
& -\int_{0}^{T} \int_{\Omega}\left[\rho u \partial_{t} \varphi+\left(\rho u^{2}+p\right) \partial_{x} \varphi\right] \mathrm{d} x \mathrm{~d} t-\int_{\Omega} \rho_{0}(x) u_{0}(x) \varphi(x, 0) \mathrm{d} x=0 \\
& p=\rho^{\gamma}
\end{aligned}
$$

Note that these relations are not sufficient to define a weak solution to the problem, since they do not imply anything about the boundary conditions. However, they allow to derive the Rankine-Hugoniot conditions; hence if we show that they are satisfied by the limit of a sequence of solutions to the discrete problem, this implies, loosely speaking, that the scheme computes correct shocks (i.e. shocks where the jumps of the unknowns and of the fluxes are linked to the shock speed by Rankine-Hugoniot conditions). This is the result we are seeking and which we state in Theorem 3.7. In order to prove this theorem, we need some definitions of interpolates of regular test functions on the primal and dual mesh.

Definition 3.6 (Interpolates on one-dimensional meshes). Let $\Omega$ be an open bounded interval of $\mathbb{R}$, let $\varphi \in$ $C_{c}^{\infty}(\Omega \times[0, T))$, and let $\mathcal{M}$ be a mesh of $\Omega$. The interpolate $\varphi_{\mathcal{M}}$ of $\varphi$ on the primal mesh $\mathcal{M}$ is defined by:

$$
\varphi_{\mathcal{M}}=\sum_{n=0}^{N-1} \sum_{K \in \mathcal{M}} \varphi_{K}^{n} \mathcal{X}_{K} \mathcal{X}_{\left[t^{n}, t^{n+1}\right)}
$$

where, for $0 \leq n \leq N$ and $K \in \mathcal{M}$, we set $\varphi_{K}^{n}=\varphi\left(x_{K}, t^{n}\right)$, with $x_{K}$ the mass center of $K$. The time and space discrete derivatives of the discrete function $\varphi_{\mathcal{M}}$ are defined by:

$$
\partial_{t} \varphi_{\mathcal{M}}=\sum_{n=0}^{N-1} \sum_{K \in \mathcal{M}} \frac{\varphi_{K}^{n+1}-\varphi_{K}^{n}}{\delta t} \mathcal{X}_{K} \mathcal{X}_{\left[t^{n}, t^{n+1}\right)} \text {, and } \partial_{x} \varphi_{\mathcal{M}}=\sum_{n=0}^{N-1} \sum_{\sigma=\overrightarrow{K \mid L} \in \mathcal{E}_{\mathrm{int}}} \frac{\varphi_{L}^{n}-\varphi_{K}^{n}}{h_{\sigma}} \mathcal{X}_{D_{\sigma}} \mathcal{X}_{\left[t^{n}, t^{n+1}\right)}
$$

Let $\varphi_{\mathcal{E}}$ be an interpolate of $\varphi$ on the dual mesh, defined by:

$$
\varphi_{\mathcal{E}}=\sum_{n=0}^{N-1} \sum_{\sigma \in \mathcal{E}} \varphi_{\sigma}^{n} \mathcal{X}_{D_{\sigma}} \mathcal{X}_{\left[t^{n}, t^{n+1}\right)}
$$


where, for $1 \leq n \leq N$ and $\sigma \in \mathcal{E}$, we set $\varphi_{\sigma}^{n}=\varphi\left(x_{\sigma}, t^{n}\right)$, with $x_{\sigma}$ the abscissa of the interface $\sigma$. We also define the time and space discrete derivatives of this discrete function by:

$$
\check{\partial}_{t} \varphi_{\mathcal{E}}=\sum_{n=0}^{N-1} \sum_{\sigma \in \mathcal{E}} \frac{\varphi_{\sigma}^{n+1}-\varphi_{\sigma}^{n}}{\delta t} \mathcal{X}_{D_{\sigma}} \mathcal{X}_{\left[t^{n}, t^{n+1}\right)} \text {, and } ð_{x} \varphi_{\mathcal{E}}=\sum_{n=0}^{N-1} \sum_{K=\left[\overrightarrow{\left.\sigma \sigma^{\prime}\right]}\right.} \frac{\varphi_{\sigma^{\prime}}^{n}-\varphi_{\sigma}^{n}}{h_{K}} \mathcal{X}_{K} \mathcal{X}_{\left[t^{n}, t^{n+1}\right)} \text {. }
$$

Finally, we define $\check{\partial}_{x} \varphi_{\mathcal{M}, \mathcal{E}}$ by:

$$
\partial_{x} \varphi_{\mathcal{M}, \mathcal{E}}=\sum_{n=0}^{N-1} \sum_{K=\left[\overrightarrow{\left.\sigma \sigma^{\prime}\right]} \in \mathcal{M}\right.} \frac{\varphi_{K}^{n}-\varphi_{\sigma}^{n}}{h_{K} / 2} \mathcal{X}_{D_{K, \sigma}} \mathcal{X}_{\left[t^{n}, t^{n+1}\right)},+\frac{\varphi_{\sigma^{\prime}}^{n}-\varphi_{K}^{n}}{h_{K} / 2} \mathcal{X}_{D_{K, \sigma^{\prime}}} \mathcal{X}_{\left[t^{n}, t^{n+1}\right)}
$$

Theorem 3.7 (Consistency of the one-dimensional implicit scheme). Let $\Omega$ be an open bounded interval of $\mathbb{R}$. We suppose that the initial data satisfies $\rho_{0} \in \mathrm{L}^{\infty}(\Omega), 1 / \rho_{0} \in \mathrm{L}^{\infty}(\Omega)$ and $u_{0} \in \mathrm{L}^{\infty}(\Omega)$. Let $\left(\mathcal{M}^{(m)}, \delta t^{(m)}, \nu^{(m)}\right)_{m \in \mathbb{N}}$ be a regular sequence of discretizations in the sense of Definition 3.5, and $\left(\rho^{(m)}, p^{(m)}, u^{(m)}\right)_{m \in \mathbb{N}}$ be the corresponding sequence of solutions. We suppose that this sequence converges in $\mathrm{L}^{p}(\Omega \times(0, T))^{3}$, for $1 \leq p<+\infty$, to $(\bar{\rho}, \bar{p}, \bar{u}) \in \mathrm{L}^{\infty}(\Omega \times(0, T))^{3}$. We suppose in addition that both sequences $\left(\rho^{(m)}\right)_{m \in \mathbb{N}}$ and $\left(1 / \rho^{(m)}\right)_{m \in \mathbb{N}}$ are uniformly bounded in $\mathrm{L}^{\infty}(\Omega \times(0, T))$.

Then the limit $(\bar{\rho}, \bar{p}, \bar{u})$ satisfies the system (3.28).

Proof. With the assumed convergence for the sequence of solutions, the limit clearly satisfies the equation of state (note that in reality this is the difficult point to prove with the estimates at hand, see e.g. [12]). The proof of this theorem is thus obtained by passing to the limit in the scheme, first for the mass balance equation and then for the momentum balance equation. Thanks to the assumption on the initial condition, the stability estimate of Proposition 3.3 is uniform with respect to $m$, and thus provides uniform bounds for some space translates of the solution (see the expression (3.22) of the remainder term), which are used all along the proof. In particular, using in addition the assumption that both sequences $\left(\rho^{(m)}\right)_{m \in \mathbb{N}}$ and $\left(1 / \rho^{(m)}\right)_{m \in \mathbb{N}}$ are uniformly bounded in $\mathrm{L}^{\infty}(\Omega \times(0, T))$, exploiting the last part of the remainder term, we get the following weak $\mathrm{B} V$ estimate for $\rho$ :

$$
\forall m \in \mathbb{N}, \quad \sum_{n=0}^{N^{(m)}-1} \delta t \sum_{\sigma=K \mid L \in \mathcal{E}_{\mathrm{int}}^{(m)}}\left|\left(u^{(m)}\right)_{\sigma}^{n+1}\right|\left[\left(\rho^{(m)}\right)_{K}^{n+1}-\left(\rho^{(m)}\right)_{L}^{n+1}\right]^{2} \leq C,
$$

where $C$ stands for a real number which is independent of $m$.

Mass balance equation - Let $\varphi \in C_{c}^{\infty}(\Omega \times[0, T))$. Let $m \in \mathbb{N}, \mathcal{M}^{(m)}, \delta t^{(m)}$ and $\nu^{(m)}$ be given. Dropping for short the superscript ${ }^{(m)}$, let $\varphi_{\mathcal{M}}$ be the interpolate of $\varphi$ on the primal mesh and let $\partial_{t} \varphi_{\mathcal{M}}$ and $\partial_{x} \varphi_{\mathcal{M}}$ be its time and space discrete derivatives in the sense of Definition 3.6. Thanks to the regularity of $\varphi$, these functions respectively converge in $\mathrm{L}^{r}\left(\Omega \times(0, T)\right.$ ), for $r \geq 1$ (including $r=+\infty$ ), to $\varphi, \partial_{t} \varphi$ and $\partial_{x} \varphi$ respectively. In addition, $\varphi_{\mathcal{M}^{(m)}}(\cdot, 0)$ converges to $\varphi(\cdot, 0)$ in $\operatorname{L}^{r}(\Omega)$ for $r \geq 1$ as $m \rightarrow+\infty$. Since the support of $\varphi$ is compact in $\Omega \times[0, T)$, for $m$ large enough, $\varphi_{\mathcal{M}^{(m)}}$ vanishes at the boundary cells and at the final time; hereafter, we systematically assume that this indeed the case.

Let us multiply the discrete mass balance equation (3.24b) by $\delta t \varphi_{K}^{n}$, and sum the result on $n \in\{0, \ldots, N-1\}$ and $K \in \mathcal{M}$, to obtain $T_{1}^{(m)}+T_{2}^{(m)}=0$ with

$$
T_{1}^{(m)}=\sum_{n=0}^{N-1} \sum_{K \in \mathcal{M}}|K|\left(\rho_{K}^{n+1}-\rho_{K}^{n}\right) \varphi_{K}^{n}, \quad T_{2}^{(m)}=\sum_{n=0}^{N-1} \delta t \sum_{K=\left[\overrightarrow{\sigma \sigma^{\prime}}\right] \in \mathcal{M}}\left[F_{\sigma^{\prime}}^{n+1}-F_{\sigma}^{n+1}\right] \varphi_{K}^{n} .
$$


(In the above expressions and in the remainder of the proof, we whall omit the superscript ${ }^{(m)}$ in the summations for the sake of clarity.) Reordering the sums in $T_{1}^{(m)}$ yields:

$$
\begin{aligned}
T_{1}^{(m)} & =-\sum_{n=0}^{N-1} \delta t \sum_{K \in \mathcal{M}}|K| \rho_{K}^{n+1} \frac{\varphi_{K}^{n+1}-\varphi_{K}^{n}}{\delta t}-\sum_{K \in \mathcal{M}}|K| \rho_{K}^{0} \varphi_{K}^{0}, \\
& =-\int_{0}^{T} \int_{\Omega} \rho^{(m)} \partial_{t} \varphi_{\mathcal{M}^{(m)}} \mathrm{d} x \mathrm{~d} t-\int_{\Omega} \rho_{0}(x) \varphi_{\mathcal{M}^{(m)}}(x, 0) \mathrm{d} x .
\end{aligned}
$$

Since, by assumption, the sequence of discrete solutions converges in $\mathrm{L}^{r}(\Omega \times(0, T))$ for $r \geq 1$, we get:

$$
\lim _{m \longrightarrow+\infty} T_{1}^{(m)}=-\int_{0}^{T} \int_{\Omega} \bar{\rho} \partial_{t} \varphi \mathrm{d} x \mathrm{~d} t-\int_{\Omega} \rho_{0}(x) \varphi(x, 0) \mathrm{d} x .
$$

Reordering the sums in $T_{2}^{(m)}$, we get:

$$
T_{2}^{(m)}=-\sum_{n=0}^{N-1} \delta t \sum_{\sigma=\overrightarrow{K \mid L} \in \mathcal{E}_{\mathrm{int}}}\left|D_{\sigma}\right| \rho_{\sigma}^{n+1} u_{\sigma}^{n+1} \frac{\varphi_{L}^{n}-\varphi_{K}^{n}}{h_{\sigma}}
$$

where $h_{\sigma}$ (which is equal to $\left|D_{\sigma}\right|$ ) is by definition equal to $\left|x_{L}-x_{K}\right|$ and we recall that $\rho_{\sigma}^{n+1}$ is the upwind approximation of $\rho^{n+1}$ at the face $\sigma$. Using the fact that $\left|D_{\sigma}\right|=(|K|+|L|) / 2$, we may write $T_{2}^{(m)}=\mathcal{T}_{2}^{(m)}+\mathcal{R}_{2}^{(m)}$ with:

$$
\begin{aligned}
\mathcal{T}_{2}^{(m)} & =-\sum_{n=0}^{N-1} \delta t \sum_{\sigma=\overrightarrow{K \mid L} \in \mathcal{E}_{\text {int }}}\left[\frac{|K|}{2} \rho_{K}^{n+1}+\frac{|L|}{2} \rho_{L}^{n+1}\right] u_{\sigma}^{n+1} \frac{\varphi_{L}^{n}-\varphi_{K}^{n}}{h_{\sigma}}, \\
\mathcal{R}_{2}^{(m)} & =-\sum_{n=0}^{N-1} \delta t \sum_{\sigma=\overrightarrow{K \mid L} \in \mathcal{E}_{\text {int }}}\left[\frac{|K|}{2}\left(\rho_{K}^{n+1}-\rho_{L}^{n+1}\right)\left(u_{\sigma}^{n+1}\right)^{-}+\frac{|L|}{2}\left(\rho_{K}^{n+1}-\rho_{L}^{n+1}\right)\left(u_{\sigma}^{n+1}\right)^{+}\right] \frac{\varphi_{L}^{n}-\varphi_{K}^{n}}{h_{\sigma}} .
\end{aligned}
$$

Therefore we get

$$
\mathcal{T}_{2}^{(m)}=-\int_{0}^{T} \int_{\Omega} \rho^{(m)} u^{(m)} \partial_{x} \varphi_{\mathcal{M}^{(m)}} \mathrm{d} x \mathrm{~d} t, \quad \text { and } \lim _{m \longrightarrow+\infty} \mathcal{T}_{2}^{(m)}=-\int_{0}^{T} \int_{\Omega} \bar{\rho} \bar{u} \partial_{x} \varphi \mathrm{d} x \mathrm{~d} t,
$$

and the remainder term $\mathcal{R}_{2}^{(m)}$ is bounded as follows:

$$
\begin{aligned}
\left|\mathcal{R}_{2}^{(m)}\right| & \leq C_{\varphi} \sum_{n=0}^{N-1} \delta t \sum_{\sigma=K \mid L \in \mathcal{E}_{\mathrm{int}}}\left|D_{\sigma}\right|\left|\rho_{K}^{n+1}-\rho_{L}^{n+1}\right|\left|u_{\sigma}^{n+1}\right| \\
& \leq C_{\varphi} h^{1 / 2} \sum_{n=0}^{N-1} \delta t\left[\sum_{\sigma=K \mid L \in \mathcal{E}_{\mathrm{int}}}\left|u_{\sigma}^{n+1}\right|\left|\rho_{K}^{n+1}-\rho_{L}^{n+1}\right|^{2}\right]^{1 / 2}\left[\sum_{\sigma=K \mid L \in \mathcal{E}_{\mathrm{int}}}\left|D_{\sigma}\right|\left|u_{\sigma}^{n+1}\right|\right]^{1 / 2},
\end{aligned}
$$

where the notation $C_{\varphi}$ means that this real number only depends on the function $\varphi$. Thanks to the stability estimate (3.29), this term tends to zero when $m$ tends to $+\infty$.

Momentum balance equation - Let $\varphi_{\mathcal{E}}, \partial_{t} \varphi_{\mathcal{E}}$ and $\partial_{x} \varphi_{\mathcal{E}}$ be the interpolate of $\varphi$ on the dual mesh and its discrete time and space derivatives, in the sense of Definition 3.6, which converge in $\operatorname{L}^{r}(\Omega \times(0, T))$, for $r \geq 1$ (including $r=+\infty$ ), to $\varphi, \partial_{t} \varphi$ and $\partial_{x} \varphi$ respectively. 
Let us multiply the discrete momentum balance equation (3.24c) by $\delta t \varphi_{\sigma}^{n}$, and sum the result over $n \in$ $\{0, \ldots, N-1\}$ and $\sigma \in \mathcal{E}_{\text {int }}$. We obtain $T_{1}^{(m)}+T_{2}^{(m)}+T_{3}^{(m)}+T_{4}^{(m)}=0$ with:

$$
\begin{aligned}
T_{1}^{(m)} & =\sum_{n=0}^{N-1} \sum_{\sigma \in \mathcal{E}_{\text {int }}}\left|D_{\sigma}\right|\left(\rho_{D_{\sigma}}^{n+1} u_{\sigma}^{n+1}-\rho_{D_{\sigma}}^{n} u_{\sigma}^{n}\right) \varphi_{\sigma}^{n}, \\
T_{2}^{(m)} & =\sum_{n=0}^{N-1} \delta t \sum_{\sigma=\overrightarrow{K \mid L} \in \mathcal{E}_{\text {int }}}\left[F_{L}^{n+1} u_{L}^{n+1}-F_{K}^{n+1} u_{K}^{n+1}\right] \varphi_{\sigma}^{n}, \\
T_{3}^{(m)} & =\sum_{n=0}^{N-1} \delta t \sum_{\sigma=\overrightarrow{K \mid L} \in \mathcal{E}_{\text {int }}}\left(p_{L}^{n+1}-p_{K}^{n+1}\right) \varphi_{\sigma}^{n}, \\
T_{4}^{(m)} & =\sum_{n=0}^{N-1} \delta t \sum_{\sigma \in \mathcal{E}_{\text {int }}}\left[\sum_{K=\left[\sigma \sigma^{\prime}\right]} \frac{\nu}{h_{K}}\left(u_{\sigma}^{n+1}-u_{\sigma^{\prime}}^{n+1}\right)\right] \varphi_{\sigma}^{n} .
\end{aligned}
$$

Thanks to the definition (3.26) of the density on the dual mesh $\rho_{D_{\sigma}}$, reordering the sums, we get for $T_{1}^{(m)}$ :

$$
T_{1}^{(m)}=-\sum_{n=0}^{N-1} \delta t \sum_{\sigma=K \mid L \in \mathcal{E}_{\mathrm{int}}}\left[\frac{|K|}{2} \rho_{K}^{n+1}+\frac{|L|}{2} \rho_{L}^{n+1}\right] u_{\sigma}^{n+1} \frac{\varphi_{\sigma}^{n+1}-\varphi_{\sigma}^{n}}{\delta t}-\sum_{\sigma=K \mid L \in \mathcal{E}_{\mathrm{int}}}\left[\frac{|K|}{2} \rho_{K}^{0}+\frac{|L|}{2} \rho_{L}^{0}\right] u_{\sigma}^{0} \varphi_{\sigma}^{0} .
$$

Therefore:

$$
T_{1}^{(m)}=-\int_{0}^{T} \int_{\Omega} \rho^{(m)} u^{(m)} \varlimsup_{t} \varphi_{\mathcal{M}^{(m)}} \mathrm{d} x \mathrm{~d} t-\int_{\Omega}\left(\rho^{(m)}\right)^{0}(x)\left(u^{(m)}\right)^{0}(x) \varphi_{\mathcal{M}^{(m)}}(x, 0) \mathrm{d} x .
$$

From the definition (3.24a) of the initial conditions and the assumed regularity of $\rho_{0}$ and $u_{0}$, the sequences $\left(\left(\rho^{(m)}\right)^{0}\right)$ and $\left.\left(u^{(m)}\right)^{0}\right)$ converge in $\mathrm{L}^{r}(\Omega)$, for $1 \leq r<+\infty$, to $\rho_{0}$ and $u_{0}$ respectively. From the convergence assumption for the sequence of discrete solutions, we thus get:

$$
\lim _{m \longrightarrow+\infty} T_{1}^{(m)}=-\int_{0}^{T} \int_{\Omega} \bar{\rho} \bar{u} \partial_{t} \varphi \mathrm{d} x \mathrm{~d} t-\int_{\Omega} \rho_{0}(x) u_{0}(x) \varphi(x, 0) \mathrm{d} x .
$$

Let us now turn to $T_{2}^{(m)}$. From the expression (3.26) of the fluxes $F_{K}$ and the values $u_{K}$, reordering the sums, we get:

$$
T_{2}^{(m)}=-\frac{1}{4} \sum_{n=0}^{N-1} \delta t \sum_{K=\left[\overrightarrow{\sigma \sigma^{\prime}}\right] \in \mathcal{M}}\left(\rho_{\sigma}^{n+1} u_{\sigma}^{n+1}+\rho_{\sigma^{\prime}}^{n+1} u_{\sigma^{\prime}}^{n+1}\right)\left(u_{\sigma}^{n+1}+u_{\sigma^{\prime}}^{n+1}\right)\left(\varphi_{\sigma^{\prime}}^{n}-\varphi_{\sigma}^{n}\right)
$$

which we write $T_{2}^{(m)}=\mathcal{T}_{2}^{(m)}+\mathcal{R}_{2}^{(m)}$ with:

$$
\mathcal{T}_{2}^{(m)}=-\frac{1}{2} \sum_{n=0}^{N-1} \delta t \sum_{K=\left[\overrightarrow{\sigma \sigma^{\prime}}\right] \in \mathcal{M}} \rho_{K}^{n+1}\left[\left(u_{\sigma}^{n+1}\right)^{2}+\left(u_{\sigma^{\prime}}^{n+1}\right)^{2}\right]\left(\varphi_{\sigma^{\prime}}^{n}-\varphi_{\sigma}^{n}\right)
$$


This term reads:

$$
\mathcal{T}_{2}^{(m)}=-\int_{0}^{T} \int_{\Omega} \rho^{(m)}\left(u^{(m)}\right)^{2} \partial_{x} \varphi_{\mathcal{E}} \mathrm{d} x \mathrm{~d} t, \text { and so } \lim _{m \longrightarrow+\infty} \mathcal{T}_{2}^{(m)}=-\int_{0}^{T} \int_{\Omega} \bar{\rho} \bar{u}^{2} \partial_{x} \varphi \mathrm{d} x \mathrm{~d} t .
$$

The remainder term $\mathcal{R}_{2}^{(m)}$ reads:

$\mathcal{R}_{2}^{(m)}=-\frac{1}{4} \sum_{n=0}^{N-1} \delta t \sum_{K=\left[\overrightarrow{\sigma \sigma^{\prime}}\right] \in \mathcal{M}}\left[\left(\rho_{\sigma}^{n+1} u_{\sigma}^{n+1}+\rho_{\sigma^{\prime}}^{n+1} u_{\sigma^{\prime}}^{n+1}\right)\left(u_{\sigma}^{n+1}+u_{\sigma^{\prime}}^{n+1}\right)-2 \rho_{K}^{n+1}\left(\left(u_{\sigma}^{n+1}\right)^{2}+\left(u_{\sigma^{\prime}}^{n+1}\right)^{2}\right)\right]\left(\varphi_{\sigma^{\prime}}^{n}-\varphi_{\sigma}^{n}\right)$.

Expanding the quantity $2 \rho_{K}^{n+1}\left(\left(u_{\sigma}^{n+1}\right)^{2}+\left(u_{\sigma^{\prime}}^{n+1}\right)^{2}\right)$ thanks to the identity $2\left(a^{2}+b^{2}\right)=(a+b)^{2}+(a-b)^{2}$, we get $\mathcal{R}_{2}^{(m)}=\mathcal{R}_{2,1}^{(m)}+\mathcal{R}_{2,2}^{(m)}$ :

$$
\begin{aligned}
& \mathcal{R}_{2,1}^{(m)}=-\frac{1}{4} \sum_{n=0}^{N-1} \delta t \sum_{K=\left[\overrightarrow{\left.\sigma \sigma^{\prime}\right]}\right.}\left[\left(\left(\rho_{\sigma}^{n+1}-\rho_{K}^{n+1}\right) u_{\sigma}^{n+1}+\left(\rho_{\sigma^{\prime}}^{n+1}-\rho_{K}^{n+1}\right) u_{\sigma^{\prime}}^{n+1}\right)\left(u_{\sigma}^{n+1}+u_{\sigma^{\prime}}^{n+1}\right)\right]\left(\varphi_{\sigma^{\prime}}^{n}-\varphi_{\sigma}^{n}\right), \\
& \mathcal{R}_{2,2}^{(m)}=\frac{1}{4} \sum_{n=0}^{N-1} \delta t \sum_{K=\left[\overrightarrow{\sigma \sigma^{\prime}}\right] \in \mathcal{M}} \rho_{K}^{n+1}\left(u_{\sigma}^{n+1}-u_{\sigma^{\prime}}^{n+1}\right)^{2}\left(\varphi_{\sigma^{\prime}}^{n}-\varphi_{\sigma}^{n}\right) .
\end{aligned}
$$

First we study $\mathcal{R}_{2,1}^{(m)}$. Thanks to the definition (3.3) of the upwind value $\rho_{\sigma}^{n+1}$, reordering the sum by faces, we get that:

$$
\left|\mathcal{R}_{2,1}^{(m)}\right|=\frac{1}{4}\left|\sum_{n=0}^{N-1} \delta t \sum_{\substack{\sigma \in \mathcal{E}_{\text {int }}, \sigma=L \rightarrow K, K=\left[\sigma \sigma^{\prime}\right]}}\left(\rho_{L}^{n+1}-\rho_{K}^{n+1}\right) u_{\sigma}^{n+1}\left(u_{\sigma}^{n+1}+u_{\sigma^{\prime}}^{n+1}\right)\left(\varphi_{\sigma}^{n}-\varphi_{\sigma^{\prime}}^{n}\right)\right|,
$$

where the notation $L \rightarrow K$ means that the flow is going from $L$ to $K$, or, in other words, that if $u_{\sigma}^{n+1} \geq 0$ (resp. $\left.u_{\sigma}^{n+1} \leq 0\right)$, the cells $K$ and $L$ are chosen such that $\sigma=\overrightarrow{L \mid K}\left(\right.$ resp. $\sigma=\overrightarrow{K \mid L}$ ). Since $\left|\varphi_{\sigma}^{n}-\varphi_{\sigma^{\prime}}^{n}\right| \leq C_{\varphi}|K| \leq$ $C_{\varphi}\left(\left|D_{\sigma}\right|+\left|D_{\sigma^{\prime}}\right|\right)$, we get:

$$
\left|\mathcal{R}_{2,1}^{(m)}\right| \leq \frac{C_{\varphi}}{4} \sum_{n=0}^{N-1} \delta t \sum_{\substack{\sigma \in \mathcal{E}_{\text {int }}, \sigma=L \rightarrow K, K=\left[\sigma \sigma^{\prime}\right]}}\left(\left|D_{\sigma}\right|+\left|D_{\sigma^{\prime}}\right|\right)\left|\rho_{L}^{n+1}-\rho_{K}^{n+1}\right|\left|u_{\sigma}^{n+1}\right|\left|u_{\sigma}^{n+1}+u_{\sigma^{\prime}}^{n+1}\right| .
$$

Therefore, by the Cauchy-Schwarz inequality, we get:

$$
\begin{aligned}
\left|\mathcal{R}_{2,1}^{(m)}\right| & \leq \frac{C_{\varphi}}{4} h^{1 / 2} \sum_{n=0}^{N-1} \delta t\left[\sum_{\sigma=K \mid L \in \mathcal{E}_{\mathrm{int}}}\left|u_{\sigma}^{n+1}\right|\left(\rho_{L}^{n+1}-\rho_{K}^{n+1}\right)^{2}\right]^{1 / 2} \\
& \times\left[\sum_{\substack{\sigma \in \mathcal{E}_{\text {int }}, \sigma=L \rightarrow K, K=\left[\sigma \sigma^{\prime}\right]}}\left(\left|D_{\sigma}\right|+\left|D_{\sigma^{\prime}}\right|\right)\left|u_{\sigma}^{n+1}\right|\left(u_{\sigma}^{n+1}+u_{\sigma^{\prime}}^{n+1}\right)^{2}\right]^{1 / 2} .
\end{aligned}
$$

Since the ratio of the size of two neighboring cells is bounded by the regularity assumption on the mesh (item (ii) of Def. 3.5), we get from the estimate (3.29) on the solution:

$$
\left|\mathcal{R}_{2,1}^{(m)}\right| \leq C h^{1 / 2}\left\|u^{(m)}\right\|_{L^{3}(\Omega \times(0, T))}^{3 / 2},
$$


where the real number $C$ is independent of $m$ and therefore $\mathcal{R}_{2,1}^{(m)}$ tends to zero when $m$ tends to $+\infty$. For $\mathcal{R}_{2,2}^{(m)}$, we have, thanks to the estimate (3.21):

$$
\left|\mathcal{R}_{2,2}^{(m)}\right| \leq C_{\varphi} h^{2} \sum_{n=0}^{N-1} \delta t \sum_{K \in \mathcal{M}} \rho_{K}^{n+1} \frac{1}{h_{K}}\left(u_{\sigma}^{n+1}-u_{\sigma^{\prime}}^{n+1}\right)^{2} \leq C \frac{h^{2}}{\nu^{(m)}}\left\|\rho^{(m)}\right\|_{L^{\infty}(\Omega \times(0, T))},
$$

where $C>0$ does not depend on $m$; therefore, this term also tends to zero when $m$ tends to $+\infty$, since, by assumption, $h^{2} / \nu^{(m)}$ tends to zero.

We turn to the term $T_{3}^{(m)}$ :

$$
T_{3}^{(m)}=-\sum_{n=0}^{N-1} \delta t \sum_{K=\left[\overrightarrow{\left.\sigma \sigma^{\prime}\right]} \in \mathcal{M}\right.}|K| p_{K}^{n+1} \frac{\varphi_{\sigma^{\prime}}^{n}-\varphi_{\sigma}^{n}}{h_{K}}=-\int_{0}^{T} \int_{\Omega} p^{(m)} \rtimes_{x} \varphi_{\mathcal{E}} \mathrm{d} x \mathrm{~d} t
$$

and therefore,

$$
\lim _{m \longrightarrow+\infty} T_{3}^{(m)}=-\int_{0}^{T} \int_{\Omega} \bar{p} \partial_{x} \varphi \mathrm{d} x \mathrm{~d} t .
$$

Let us finally study $T_{4}^{(m)}$. Reordering the sums, we get:

$$
T_{4}^{(m)}=\sum_{n=0}^{N-1} \delta t \sum_{K=\left[\overrightarrow{\left.\sigma \sigma^{\prime}\right]}\right] \in \mathcal{M}} \frac{\nu^{(m)}}{h_{K}}\left(u_{\sigma}^{n+1}-u_{\sigma^{\prime}}^{n+1}\right)\left(\varphi_{\sigma}^{n}-\varphi_{\sigma^{\prime}}^{n}\right)
$$

The Cauchy-Schwarz inequality yields:

$$
\left|T_{4}^{(m)}\right| \leq\left[\sum_{n=0}^{N-1} \delta t \sum_{K=\left[\overrightarrow{\left.\sigma \sigma^{\prime}\right]} \in \mathcal{M}\right.} \frac{\nu^{(m)}}{h_{K}}\left(u_{\sigma}^{n+1}-u_{\sigma^{\prime}}^{n+1}\right)^{2}\right]^{1 / 2}\left[\sum_{n=0}^{N-1} \delta t \sum_{K=\left[\overrightarrow{\left.\sigma \sigma^{\prime}\right]} \in \mathcal{M}\right.} \frac{\nu^{(m)}}{h_{K}}\left(\varphi_{\sigma}^{n}-\varphi_{\sigma^{\prime}}^{n}\right)^{2}\right]^{1 / 2},
$$

and thus, in view of the estimate (3.21), this term tends to zero when $\nu^{(m)}$ tends to zero.

Conclusion - Gathering the limits of all the terms of the mass and momentum balance equations concludes the proof.

Remark 3.8 (Sharper bounds and convergence assumptions). The convergence properties and bounds assumed for the solution have been chosen so as to match what may be observed in the theoretical works on compressible Navier-Stokes equations $[13,44,53]$. Note that they are weaker than the assumptions of the Lax-Wendroff theorem for colocated finite volume schemes on hyperbolic systems, see e.g. [11], Theorem 5.3. However, examining the proof of Theorem 3.7, we observe that what we really need is that the sequences $\rho^{(m)} u^{(m)}, \rho^{(m)}\left(u^{(m)}\right)^{2}$, $p^{(m)} u^{(m)}$ converge in the distribution sense to $\bar{\rho} \bar{u}, \bar{\rho} \bar{u}^{2}$ and $\bar{p} \bar{u}$ respectively, that $\left(\rho^{(m)}\right)^{\gamma}$ converge a.e. to $\bar{\rho}^{\gamma}$, and that the sequence $\left(u^{(m)}\right)_{m \in \mathbb{N}}$ be bounded in $\mathrm{L}^{3}(\Omega \times(0, T))$. The required second assumption for $\left(\nu^{(m)}\right)_{m \in \mathbb{N}}$ is in fact:

$$
\lim _{m \rightarrow+\infty} \frac{\left(h^{(m)}\right)^{2}}{\nu^{(m)}}\left\|\rho^{(m)}\right\|_{L^{\infty}(\Omega \times(0, T))}=0,
$$

and may be verified, for instance supposing a relation between $\delta t^{(m)}$ and $h^{(m)}$ and invoking inverse inequalities, with milder estimates on $\left(\rho^{(m)}\right)_{m \in \mathbb{N}}$. Finally, the bound of $\left(1 / \rho^{(m)}\right)_{m \in \mathbb{N}}$ in $\mathrm{L}^{\infty}(\Omega \times(0, T)$ ) (which, loosely speaking, means that the appearance of void is excluded) is needed to obtain the weak-B $V$ estimate:

$$
\lim _{m \rightarrow+\infty} h^{(m)} \sum_{n=1}^{N} \sum_{\sigma=K \mid L \in \mathcal{E}_{\text {int }}}\left|u_{\sigma}^{n}\right|\left(\rho_{K}^{n}-\rho_{L}^{n}\right)^{2}=0
$$


from the "weighted weak-BV estimate" (3.21):

$$
\lim _{m \rightarrow+\infty} h^{(m)} \sum_{n=1}^{N} \sum_{\sigma=K \mid L \in \mathcal{E}_{\mathrm{int}}}\left(\rho_{\sigma, \gamma}^{n}\right)^{\gamma-2}\left|u_{\sigma}^{n}\right|\left(\rho_{K}^{n}-\rho_{L}^{n}\right)^{2}=0,
$$

where we recall that $\rho_{\sigma, \gamma}^{n}$ is equal to either $\rho_{K}^{n}$ or $\rho_{L}^{n}$. This assumption is thus useless for $\gamma \leq 2$. For $\gamma>2$, in the case of a non-vanishing viscosity, equation (3.32) may perhaps be derived by using the density itself as test function in the discrete mass balance equation, and invoking a control of the divergence of the velocity (from the momentum balance diffusion term), see ([12], Prop. 5.5) for such a computation in the steady case.

Remark 3.9 (Less sharp bounds and more general meshes). The assumption that the ratio of the size of two neighboring cells is bounded, i.e. assumption (ii) of Definition 3.5, is only used to derive (3.31) from (3.30), which allows to conclude that the remainder term $\mathcal{R}_{2,1}^{(m)}$ tends to zero invoking a control of the velocity only in $\mathrm{L}^{3}(\Omega \times(0, T))$. If we choose to use a uniform bound in $\mathrm{L}^{\infty}(\Omega \times(0, T))$ for the sequence of approximate solutions, we may replace $(3.31)$ by

$$
\left|\mathcal{R}_{2,1}^{(m)}\right| \leq C h^{1 / 2}\left\|u^{(m)}\right\|_{L^{\infty}(\Omega \times(0, T))}^{3 / 2},
$$

and assumption (ii) is useless.

We now turn to the entropy condition. Let us first recall that $\eta=\rho E_{k}+\mathcal{H}(\rho)$ is an entropy of the continuous problem (1.1), in the sense that if we sum the formal kinetic energy (3.13) and elastic potential balance (3.18), we get:

$$
\partial_{t} \eta+\partial_{x}((\eta+p) u)=0
$$

In fact, in order to avoid to invoke unrealistic regularity assumption, such a computation should be done on regularized equations (obtained by adding diffusion perturbation terms), and, when making these regularization terms tend to zero, positive measures appear at the left-hand-side of (3.33), so that we get in the distribution sense:

$$
\partial_{t} \eta+\partial_{x}((\eta+p) u) \leq 0
$$

An entropy solution to (1.1) is thus required to satisfy, for any $\varphi \in \mathrm{C}_{c}^{\infty}(\Omega \times[0, T)), \varphi \geq 0$ :

$$
\int_{0}^{T} \int_{\Omega}\left[\eta \partial_{t} \varphi+(\eta+p) u \partial_{x} \varphi\right] \mathrm{d} \boldsymbol{x} \mathrm{d} t+\int_{\Omega} \eta_{0}(\boldsymbol{x}) \varphi(\boldsymbol{x}, 0) \mathrm{d} \boldsymbol{x} \geq 0
$$

where $\eta_{0}=\frac{1}{2} \rho_{0} u_{0}^{2}+\mathcal{H}\left(\rho_{0}\right)$.

Theorem 3.10 (Entropy consistency, implicit scheme). Under the assumptions of Theorem $3.7,(\bar{\rho}, \bar{p}, \bar{u})$ satisfies the entropy condition (3.35).

Proof. Let $\varphi \in \mathrm{C}_{c}^{\infty}(\Omega \times[0, T)), \varphi \geq 0$. Using the notations introduced in Definition 3.6, we multiply the kinetic balance equation (3.14) by $\varphi_{\sigma}^{n}$, and the elastic potential balance (3.19) by $\varphi_{K}^{n}$, sum over the faces and cells respectively, to get

$$
\sum_{\mathcal{E} \in \mathcal{E}_{\text {int }}} T_{\sigma}^{n+1} \varphi_{\sigma}^{n}+\sum_{K \in \mathcal{M}} T_{K}^{n+1} \varphi_{K}^{n}=-\sum_{\mathcal{E} \in \mathcal{E}_{\text {int }}} R_{\sigma}^{n+1} \varphi_{\sigma}^{n}-\sum_{K \in \mathcal{M}} R_{K}^{n+1} \varphi_{K}^{n},
$$

where, for $\sigma=\overrightarrow{K \mid L}, K=\left[\overrightarrow{\sigma^{\prime} \sigma}\right]$ and $L=\left[\overrightarrow{\sigma \sigma^{\prime \prime}}\right]$,

$$
T_{\sigma}^{n+1}=\frac{1}{2} \frac{\left|D_{\sigma}\right|}{\delta t}\left[\rho_{D_{\sigma}}^{n+1}\left(u_{\sigma}^{n+1}\right)^{2}-\rho_{D_{\sigma}}^{n}\left(u_{\sigma}^{n}\right)^{2}\right]+\frac{1}{2} F_{L}^{n+1} u_{\sigma}^{n+1} u_{\sigma^{\prime \prime}}^{n+1}-\frac{1}{2} F_{K}^{n+1} u_{\sigma}^{n+1} u_{\sigma^{\prime}}^{n+1}+\left(p_{L}^{n+1}-p_{K}^{n+1}\right) u_{\sigma}^{n+1},
$$


for $K=\left[\overrightarrow{\sigma \sigma^{\prime}}\right]$,

$$
T_{K}^{n+1}=\frac{|K|}{\delta t}\left[\mathcal{H}\left(\rho_{K}^{n+1}\right)-\mathcal{H}\left(\rho_{K}^{n}\right)\right]+u_{\sigma^{\prime}}^{n+1} \mathcal{H}\left(\rho_{\sigma^{\prime}}^{n+1}\right)-u_{\sigma}^{n+1} \mathcal{H}\left(\rho_{\sigma}^{n+1}\right)+p_{K}^{n+1}\left(u_{\sigma^{\prime}}^{n+1}-u_{\sigma}^{n+1}\right),
$$

and the quantities $R_{\sigma}^{n+1}$ and $R_{K}^{n+1}$ are given by (the one-dimensional version of) equations (3.15) and (3.20) respectively.

The discrete weak form of the entropy balance is obtained by integrating in time (i.e. summing over the time steps) equation (3.36). We obtain $T_{1}^{(m)}+T_{2}^{(m)}+T_{3}^{(m)}+T_{4}^{(m)}+T_{5}^{(m)}+R^{(m)}=0$ with:

$$
\begin{aligned}
& T_{1}^{(m)}=\frac{1}{2} \sum_{n=0}^{N-1} \sum_{\sigma \in \mathcal{E}_{\text {int }}}\left|D_{\sigma}\right|\left[\rho_{D_{\sigma}}^{n+1}\left(u_{\sigma}^{n+1}\right)^{2}-\rho_{D_{\sigma}}^{n}\left(u_{\sigma}^{n}\right)^{2}\right] \varphi_{\sigma}^{n}, \\
& T_{2}^{(m)}=\frac{1}{2} \sum_{n=0}^{N-1} \sum_{K \in \mathcal{M}}|K|\left[\mathcal{H}\left(\rho_{K}^{n+1}\right)-\mathcal{H}\left(\rho_{K}^{n}\right)\right] \varphi_{K}^{n} \\
& T_{3}^{(m)}=\frac{1}{2} \sum_{n=0}^{N-1} \delta t \sum_{\substack{\sigma=\overrightarrow{K \mid L} \in \mathcal{E}_{\text {int }}, K=\left[\overrightarrow{\left.\sigma^{\prime} \sigma\right]}, L=\left[\overrightarrow{\sigma \sigma^{\prime \prime}}\right]\right.}}\left[F_{L}^{n+1} u_{\sigma}^{n+1} u_{\sigma^{\prime \prime}}^{n+1}-F_{K}^{n+1} u_{\sigma}^{n+1} u_{\sigma^{\prime}}^{n+1}\right] \varphi_{\sigma}^{n} \\
& T_{4}^{(m)}=\sum_{n=0}^{N-1} \delta t \sum_{K=\left[\overrightarrow{\sigma \sigma^{\prime}}\right] \in \mathcal{M}}\left[u_{\sigma^{\prime}}^{n+1} \mathcal{H}\left(\rho_{\sigma^{\prime}}^{n+1}\right)-u_{\sigma}^{n+1} \mathcal{H}\left(\rho_{\sigma}^{n+1}\right)\right] \varphi_{K}^{n}, \\
& T_{5}^{(m)}=\frac{1}{2} \sum_{n=0}^{N-1} \delta t\left[\sum_{\sigma=\overrightarrow{K \mid L} \in \mathcal{E}_{\mathrm{int}}}\left(p_{L}^{n+1}-p_{K}^{n+1}\right) u_{\sigma}^{n+1} \varphi_{\sigma}^{n}+\sum_{K=\left[\overrightarrow{\sigma \sigma^{\prime}}\right] \in \mathcal{M}} p_{K}^{n+1}\left(u_{\sigma^{\prime}}^{n+1}-u_{\sigma}^{n+1}\right) \varphi_{K}^{n}\right], \\
& R^{(m)}=\frac{1}{2} \sum_{n=0}^{N-1} \delta t\left[\sum_{\sigma=\overline{K \mid L} \in \mathcal{E}_{\mathrm{int}}} R_{\sigma}^{n} \varphi_{\sigma}^{n}+\sum_{K=\left[\overrightarrow{\sigma \sigma^{\prime}}\right] \in \mathcal{M}} R_{K}^{n} \varphi_{K}^{n}\right]
\end{aligned}
$$

We first study $T_{1}^{(m)}$. Reordering the summations and then using the definition (3.26) of the density at the faces, we get:

$$
\begin{aligned}
T_{1}^{(m)} & =-\frac{1}{2} \sum_{n=0}^{N-1} \delta t \sum_{\sigma \in \mathcal{E}}\left|D_{\sigma}\right| \rho_{D_{\sigma}}^{n+1}\left(u_{\sigma}^{n+1}\right)^{2} \frac{\varphi_{\sigma}^{n+1}-\varphi_{\sigma}^{n}}{\delta t}-\frac{1}{2} \sum_{\sigma \in \mathcal{E}}\left|D_{\sigma}\right| \rho_{D_{\sigma}}^{0}\left(u_{\sigma}^{0}\right)^{2} \varphi_{\sigma}^{0} \\
& =-\frac{1}{2} \int_{0}^{T} \int_{\Omega} \rho^{(m)}\left(u^{(m)}\right)^{2} \partial_{t} \varphi_{\mathcal{E}} \mathrm{d} x \mathrm{~d} t-\frac{1}{2} \int_{\Omega}\left(\rho^{(m)}\right)^{0}(x)\left[\left(u^{(m)}\right)^{0}(x)\right]^{2} \varphi_{\mathcal{E}}(x, 0) \mathrm{d} x .
\end{aligned}
$$

By the definition (3.53a) of the initial conditions of the scheme, since both $\rho_{0}$ and $u_{0}$ are supposed to belong to $\mathrm{L}^{\infty}(\Omega),\left(\rho^{(m)}\right)^{0}$ and $\left(u^{(m)}\right)^{0}$ converge to $\rho_{0}$ and $u_{0}$ respectively in $\mathrm{L}^{r}(\Omega)$, for $r \geq 1$. Since, by assumption, the sequence of discrete solutions converges in $\operatorname{L}^{r}(\Omega \times(0, T))$ for $r \geq 1$, we can pass to the limit in the previous relation, to get:

$$
\lim _{m \longrightarrow+\infty} T_{1}^{(m)}=-\frac{1}{2} \int_{0}^{T} \int_{\Omega} \bar{\rho}(\bar{u})^{2} \partial_{t} \varphi \mathrm{d} x \mathrm{~d} t-\frac{1}{2} \int_{\Omega} \rho_{0}(x) u_{0}(x)^{2} \varphi(x, 0) \mathrm{d} x .
$$


By a similar computation, we get for $T_{2}^{(m)}$ :

$$
\begin{aligned}
T_{2}^{(m)} & =-\sum_{n=0}^{N-1} \delta t \sum_{K \in \mathcal{M}}|K| \mathcal{H}\left(\rho_{K}^{n+1}\right) \frac{\varphi_{K}^{n+1}-\varphi_{K}^{n}}{\delta t}-\sum_{\sigma \in \mathcal{E}}|K| \mathcal{H}\left(\rho_{K}^{0}\right) \varphi_{K}^{0} \\
& =-\int_{0}^{T} \int_{\Omega} \mathcal{H}\left(\rho^{(m)}\right) \grave{\partial}_{t} \varphi_{\mathcal{M}^{(m)}} \mathrm{d} x \mathrm{~d} t-\int_{\Omega} \mathcal{H}\left(\left(\rho^{(m)}\right)^{0}\right)(x) \varphi_{\mathcal{M}^{(m)}}(x, 0) \mathrm{d} x
\end{aligned}
$$

and therefore:

$$
\lim _{m \longrightarrow+\infty} T_{2}^{(m)}=-\int_{0}^{T} \int_{\Omega} \mathcal{H}(\bar{\rho}) \partial_{t} \varphi \mathrm{d} x \mathrm{~d} t-\int_{\Omega} \mathcal{H}\left(\rho_{0}\right)(x) \varphi(x, 0) \mathrm{d} x .
$$

Let us now study the kinetic energy convection term $T_{3}^{(m)}$ which reads, after reordering the summations:

$$
T_{3}^{(m)}=-\frac{1}{2} \sum_{n=0}^{N-1} \delta t \sum_{K=\left[\overrightarrow{\sigma \sigma^{\prime}}\right] \in \mathcal{M}} F_{K}^{n+1} u_{\sigma}^{n+1} u_{\sigma^{\prime}}^{n+1}\left(\varphi_{\sigma}^{n}-\varphi_{\sigma^{\prime}}^{n}\right) .
$$

Using now the definition of the mass fluxes at the dual edges, we have:

$$
T_{3}^{(m)}=-\frac{1}{4} \sum_{n=0}^{N-1} \delta t \sum_{K=\left[\overrightarrow{\sigma \sigma^{\prime}}\right] \in \mathcal{M}}\left(\rho_{\sigma}^{n+1} u_{\sigma}^{n+1}+\rho_{\sigma^{\prime}}^{n+1} u_{\sigma^{\prime}}^{n+1}\right) u_{\sigma^{\prime}}^{n+1} u_{\sigma}^{n+1}\left(\varphi_{\sigma}^{n}-\varphi_{\sigma^{\prime}}^{n}\right) .
$$

We now split $T_{3}^{(m)}=\mathcal{T}_{3}^{(m)}+\mathcal{R}_{3}^{(m)}$, where

$$
\mathcal{T}_{3}^{(m)}=-\frac{1}{4} \sum_{n=0}^{N-1} \delta t \sum_{K=\left[\overrightarrow{\sigma \sigma^{\prime}}\right] \in \mathcal{M}} \rho_{K}^{n+1}\left[\left(u_{\sigma}^{n+1}\right)^{3}+\left(u_{\sigma^{\prime}}^{n+1}\right)^{3}\right]\left(\varphi_{\sigma}^{n}-\varphi_{\sigma^{\prime}}^{n}\right)=-\frac{1}{2} \int_{0}^{T} \int_{\Omega} \rho^{(m)}\left(u^{(m)}\right)^{3} \varlimsup_{x} \varphi_{\mathcal{E}} \mathrm{d} x \mathrm{~d} t,
$$

so that

$$
\lim _{m \longrightarrow+\infty} \mathcal{T}_{3}^{(m)}=-\frac{1}{2} \int_{0}^{T} \int_{\Omega} \bar{\rho} \bar{u}^{3} \partial_{x} \varphi \mathrm{d} x \mathrm{~d} t
$$

and

$$
\mathcal{R}_{3}^{(m)}=-\frac{1}{4} \sum_{n=0}^{N-1} \delta t \sum_{K=\left[\overrightarrow{\sigma \sigma^{\prime}}\right] \in \mathcal{M}}\left[\left(\rho_{\sigma}^{n+1} u_{\sigma}^{n+1}+\rho_{\sigma^{\prime}}^{n+1} u_{\sigma^{\prime}}^{n+1}\right) u_{\sigma}^{n+1} u_{\sigma^{\prime}}^{n+1}-\rho_{K}^{n+1}\left(\left(u_{\sigma}^{n+1}\right)^{3}+\left(u_{\sigma^{\prime}}^{n+1}\right)^{3}\right)\right]\left(\varphi_{\sigma}^{n}-\varphi_{\sigma^{\prime}}^{n}\right) .
$$

Expanding the quantity $\left(u_{\sigma}^{n+1}\right)^{3}+\left(u_{\sigma^{\prime}}^{n+1}\right)^{3}$ thanks to the identity $a^{3}+b^{3}=(a+b)\left(a b+(a-b)^{2}\right)$, we obtain $\mathcal{R}_{3}^{(m)}=\mathcal{R}_{3,1}^{(m)}+\mathcal{R}_{3,2}^{(m)}$ with:

$$
\begin{aligned}
& \mathcal{R}_{3,1}^{(m)}=-\frac{1}{4} \sum_{n=0}^{N-1} \delta t \sum_{K=\left[\overrightarrow{\sigma \sigma^{\prime}}\right] \in \mathcal{M}}\left[\left(\rho_{\sigma}^{n+1}-\rho_{K}^{n+1}\right) u_{\sigma}^{n+1}+\left(\rho_{\sigma^{\prime}}^{n+1}-\rho_{K}^{n+1}\right) u_{\sigma^{\prime}}^{n+1}\right] u_{\sigma}^{n+1} u_{\sigma^{\prime}}^{n+1}\left(\varphi_{\sigma}^{n}-\varphi_{\sigma^{\prime}}^{n}\right), \\
& \mathcal{R}_{3,2}^{(m)}=\frac{1}{4} \sum_{n=0}^{N} \delta t \sum_{K=\left[\overrightarrow{\sigma \sigma^{\prime}}\right] \in \mathcal{M}} \rho_{K}^{n+1}\left(u_{\sigma}^{n+1}+u_{\sigma^{\prime}}^{n+1}\right)\left(u_{\sigma}^{n+1}-u_{\sigma^{\prime}}^{n+1}\right)^{2}\left(\varphi_{\sigma}^{n}-\varphi_{\sigma^{\prime}}^{n}\right) .
\end{aligned}
$$

Reordering the sums, the term $\mathcal{R}_{3,1}^{(m)}$ reads:

$$
\mathcal{R}_{3,1}^{(m)}=\frac{1}{4} \sum_{n=0}^{N-1} \delta t \sum_{\substack{\sigma \in \mathcal{E}_{\text {int }}, \sigma=L \rightarrow K, K=\left[\sigma \sigma^{\prime}\right]}} \varepsilon_{\sigma}^{n+1}\left(\rho_{L}^{n+1}-\rho_{K}^{n+1}\right)\left(u_{\sigma}^{n+1}\right)^{2} u_{\sigma^{\prime}}^{n+1}\left(\varphi_{\sigma}^{n}-\varphi_{\sigma^{\prime}}^{n}\right),
$$


where $\varepsilon_{\sigma}^{n+1}= \pm 1$ and the notation $L \rightarrow K$ means that the flow is going from $L$ to $K$. Thanks to the Cauchy-Schwarz inequality, we get, by the regularity of $\varphi$ :

$$
\begin{aligned}
\left|\mathcal{R}_{3,1}^{(m)}\right| \leq & C_{\varphi} h^{1 / 2}\left[\sum_{n=0}^{N-1} \delta t \sum_{\sigma=K \mid L \in \mathcal{E}_{\text {int }}}\left|u_{\sigma}^{n+1}\right|\left(\rho_{L}^{n+1}-\rho_{K}^{n+1}\right)^{2}\right]^{1 / 2} \\
\times & {\left[\sum_{n=0}^{N-1} \delta t \sum_{\substack{\sigma \in \mathcal{E}_{\text {int }}, \sigma=L \rightarrow K, K=\left[\sigma \sigma^{\prime}\right]}}|K|\left|u_{\sigma}^{n+1}\right|\left(u_{\sigma}^{n+1} u_{\sigma^{\prime}}^{n+1}\right)^{2}\right]^{1 / 2}, }
\end{aligned}
$$

and thus:

$$
\left|\mathcal{R}_{3,1}^{(m)}\right| \leq C_{\varphi} h^{1 / 2}\left\|u^{(m)}\right\|_{L^{5}(\Omega \times(0, T))}^{5 / 2} .
$$

We now turn to $\mathcal{R}_{3,2}^{(m)}$. Thanks to the regularity of $\varphi$, we get:

$$
\left|\mathcal{R}_{3,2}^{(m)}\right| \leq C_{\varphi} \frac{\left(h^{(m)}\right)^{2}}{\nu^{(m)}}\left\|\rho^{(m)}\right\|_{L^{\infty}(\Omega \times(0, T))}\left\|u^{(m)}\right\|_{L^{\infty}(\Omega \times(0, T))}^{2} \sum_{K=\left[\sigma \sigma^{\prime}\right] \in \mathcal{M}} \frac{\nu^{(m)}}{h_{K}}\left(u_{\sigma}^{n+1}-u_{\sigma^{\prime}}^{n+1}\right)^{2},
$$

and thus $\mathcal{R}_{3,2}^{(m)}$ also tends to zero when $m$ tends to $+\infty$ as soon as the ratio $\left(h^{(m)}\right)^{2} / \nu^{(m)}$ tends to zero. As a consequence, we get that

$$
\lim _{m \rightarrow+\infty} T_{3}^{(m)}=-\frac{1}{2} \int_{0}^{T} \int_{\Omega} \bar{\rho} \bar{u}^{3} \partial_{x} \varphi \mathrm{d} x \mathrm{~d} t
$$

Expressing the mass fluxes as a function of the unknowns in $T_{4}^{(m)}$ and reordering the sums, we get:

$$
T_{4}^{(m)}=-\sum_{n=0}^{N-1} \delta t \sum_{\sigma=\overrightarrow{K \mid L} \in \mathcal{E}_{\text {int }}} \mathcal{H}\left(\rho_{\sigma}^{n+1}\right) u_{\sigma}^{n+1}\left(\varphi_{K}^{n}-\varphi_{L}^{n}\right) .
$$

Let us write $T_{4}^{(m)}=\mathcal{T}_{4}^{(m)}+\mathcal{R}_{4}^{(m)}$, with, thanks to the definition of the upwind density (3.3) at the face:

$$
\begin{aligned}
\mathcal{T}_{4}^{(m)} & =-\sum_{n=0}^{N-1} \delta t \sum_{\sigma=\overrightarrow{K \mid L} \in \mathcal{E}_{\mathrm{int}}}\left[\left|D_{K, \sigma}\right| \mathcal{H}\left(\rho_{K}^{n+1}\right)+\left|D_{L, \sigma}\right| \mathcal{H}\left(\rho_{L}^{n+1}\right)\right] u_{\sigma}^{n+1} \frac{\varphi_{K}^{n}-\varphi_{L}^{n}}{h_{\sigma}} \\
\mathcal{R}_{4}^{(m)} & =\sum_{n=0}^{N-1} \delta t \sum_{\sigma=\overrightarrow{K \mid L} \in \mathcal{E}_{\mathrm{int}}}\left|D_{L, \sigma}\right|\left[\mathcal{H}\left(\rho_{L}^{n+1}\right)-\mathcal{H}\left(\rho_{K}^{n+1}\right)\right] u_{\sigma}^{n+1} \frac{\varphi_{K}^{n}-\varphi_{L}^{n}}{h_{\sigma}}
\end{aligned}
$$

We have:

$$
\mathcal{T}_{4}^{(m)}=-\int_{0}^{T} \int_{\Omega} \mathcal{H}\left(\rho^{(m)}\right) u^{(m)} \mathrm{\partial}_{x} \varphi_{\mathcal{M}^{(m)}} \mathrm{d} x \mathrm{~d} t, \quad \text { so } \quad \lim _{m \longrightarrow+\infty} \mathcal{T}_{4}^{(m)}=-\int_{0}^{T} \int_{\Omega} \mathcal{H}(\bar{\rho}) \bar{u} \partial_{x} \varphi \mathrm{d} x \mathrm{~d} t .
$$

By the regularity of $\varphi$, we get:

$$
\left|\mathcal{R}_{4}^{(m)}\right| \leq C_{\varphi} h^{(m)} \sum_{n=0}^{N-1} \delta t \sum_{\sigma=K \mid L \in \mathcal{E}_{\text {int }}}\left|\mathcal{H}\left(\rho_{K}^{n+1}\right)-\mathcal{H}\left(\rho_{L}^{n+1}\right)\right|\left|u_{\sigma}^{n+1}\right| .
$$


Since both sequences $\left(\rho^{(m)}\right)_{m \in \mathbb{N}}$ and $\left(1 / \rho^{(m)}\right)_{m \in \mathbb{N}}$ are supposed to be uniformly bounded, we have $\mid \mathcal{H}\left(\rho_{K}^{n+1}\right)-$ $\mathcal{H}\left(\rho_{L}^{n+1}\right)|\leq C| \rho_{K}^{n+1}-\rho_{L}^{n+1} \mid$ with a constant real number $C$, and therefore $\mathcal{R}_{4}^{(m)}$ tends to zero as $h^{(m)}$. Therefore,

$$
\lim _{m \rightarrow+\infty} T_{4}^{(m)}=-\int_{0}^{T} \int_{\Omega} \mathcal{H}(\bar{\rho}) \bar{u} \partial_{x} \varphi \mathrm{d} x \mathrm{~d} t .
$$

Reordering the sums in the term $T_{5}^{(m)}$, we obtain:

$$
T_{5}^{(m)}=\sum_{n=0}^{N-1}-\delta t \sum_{K=\left[\overrightarrow{\sigma \sigma^{\prime}}\right] \in \mathcal{M}} p_{K}^{n+1}\left(u_{\sigma^{\prime}}^{n+1} \varphi_{\sigma^{\prime}}^{n}-u_{\sigma}^{n+1} \varphi_{\sigma}^{n}\right)+p_{K}^{n+1}\left(u_{\sigma}^{n+1}-u_{\sigma^{\prime}}^{n+1}\right) \varphi_{K}^{n},
$$

hence:

$$
\begin{aligned}
T_{5}^{(m)} & =-\sum_{n=0}^{N-1} \delta t \sum_{K=\left[\overrightarrow{\sigma \sigma^{\prime}}\right] \in \mathcal{M}} \frac{|K|}{2} p_{K}^{n+1} u_{\sigma}^{n+1} \frac{\varphi_{K}^{n}-\varphi_{\sigma}^{n}}{h_{K} / 2}+\frac{|K|}{2} p_{K}^{n+1} u_{\sigma^{\prime}}^{n+1} \frac{\varphi_{\sigma^{\prime}}^{n}-\varphi_{K}^{n}}{h_{K} / 2} \\
& =-\int_{0}^{T} \int_{\Omega} p^{(m)} u^{(m)} \partial_{x} \varphi_{\mathcal{M}, \mathcal{E}} \mathrm{d} x \mathrm{~d} t .
\end{aligned}
$$

and so, since $\partial_{x} \varphi_{\mathcal{M}, \mathcal{E}}$ converges to $\partial_{x} \varphi$ in $\mathrm{L}^{r}(\Omega)$, for $r \geq 1$ :

$$
\lim _{m \longrightarrow+\infty} T_{5}^{(m)}=-\int_{0}^{T} \int_{\Omega} \bar{p} \bar{u} \partial_{x} \varphi \mathrm{d} x \mathrm{~d} t .
$$

From (3.38)-(3.41), we get that

$$
\lim _{m \longrightarrow+\infty} \sum_{i=1}^{5} T_{i}^{(m)}=-\int_{0}^{T} \int_{\Omega}\left[\eta \partial_{t} \varphi+(\eta+p) u \partial_{x} \varphi\right] \mathrm{d} x \mathrm{~d} t-\int_{\Omega} \eta_{0}(x) \varphi(x, 0) \mathrm{d} x .
$$

In order to complete the proof of Theorem 3.17, there only remains to show that $\lim _{m \rightarrow+\infty} R^{(m)} \geq 0$. Since we only seek an inequality, the non-negative part of $R^{(m)}$, i.e. the first part in $R_{\sigma}^{n+1}$ and the whole term $R_{K}^{n+1}$, poses no problem, and we only have to study the terms coming from the second part of $R_{\sigma}^{n+1}$, which reads:

$$
\left(R_{\mathrm{diff}}\right)_{\sigma}^{n+1}=\left[\sum_{K=\left[\sigma \sigma^{\prime}\right]} \frac{\nu^{(m)}}{h_{K}}\left(u_{\sigma}^{n+1}-u_{\sigma^{\prime}}^{n+1}\right)\right] u_{\sigma}^{n+1} .
$$

For $0 \leq n \leq N-1$ and $K \in \mathcal{M}, K=\left[\sigma \sigma^{\prime}\right]$, let us define the quantity $Q_{K}^{n+1}$ by:

$$
Q_{K}^{n+1}=\frac{\nu^{(m)}}{h_{K}}\left(u_{\sigma}^{n+1}-u_{\sigma^{\prime}}^{n+1}\right)^{2} .
$$

We have $Q_{K}^{n+1} \geq 0$, and, reordering the summation, we get that

$$
\begin{aligned}
\mathcal{Q}^{(m)} & =\left|\sum_{n=0}^{N-1} \delta t\left[\sum_{\sigma \in \mathcal{E}_{\text {int }}} \varphi_{\sigma}^{n}\left(R_{\mathrm{diff}}\right)_{\sigma}^{n+1}-\sum_{K \in \mathcal{M}} \varphi_{K}^{n} Q_{K}^{n+1}\right]\right| \\
& =\left|\sum_{n=0}^{N-1} \delta t \sum_{K=\left[\overrightarrow{\sigma \sigma^{\prime}}\right] \in \mathcal{M}} \frac{\nu}{h_{K}}\left(u_{\sigma}^{n+1}-u_{\sigma^{\prime}}^{n+1}\right)\left[u_{\sigma}^{n+1}\left(\varphi_{\sigma}-\varphi_{K}\right)-u_{\sigma^{\prime}}^{n+1}\left(\varphi_{\sigma^{\prime}}-\varphi_{K}\right)\right]\right| .
\end{aligned}
$$


By the Cauchy-Schwarz inequality and the regularity of $\varphi$, we thus get:

$$
\begin{aligned}
\mathcal{Q}^{(m)} & \leq C_{\varphi}\left[\sum_{n=0}^{N-1} \delta t \sum_{K=\left[\overrightarrow{\sigma \sigma^{\prime}}\right] \in \mathcal{M}} \frac{\nu^{(m)}}{h_{K}}\left(u_{\sigma}^{n+1}-u_{\sigma^{\prime}}^{n+1}\right)^{2}\right]^{1 / 2} \\
& \times\left[\sum_{n=0}^{N-1} \delta t \sum_{K=\left[\overrightarrow{\sigma \sigma^{\prime}}\right] \in \mathcal{M}} \nu^{(m)}\left(\left|D_{K, \sigma}\right|\left(u_{\sigma}^{n+1}\right)^{2}+\left|D_{K, \sigma^{\prime}}\right|\left(u_{\sigma^{\prime}}^{n+1}\right)^{2}\right)\right]^{1 / 2} .
\end{aligned}
$$

From estimate (3.21), we thus get that

$$
\mathcal{Q}^{(m)} \leq C\left(\nu^{(m)}\right)^{1 / 2},
$$

where $C>0$ only depends on $\varphi$, on $C_{0}$ and on the assumed bounds on the solution. Since $\nu^{(m)}$ tends to 0 with $m$, we then obtain that $\lim _{m \rightarrow+\infty} R^{(m)} \geq 0$, which concludes the proof.

\subsection{The pressure correction scheme for the barotropic equations}

The implicit scheme which we studied in the previous section is easy to write, but difficult to implement in practice, because of the large nonlinear systems to be solved at the algebraic level. Pressure correction methods are based on the idea that one may compute the velocity and the pressure in a sequential way, thus yielding a more practical scheme. The algorithm presented in this section is implemented in the open-source software component library for fluid flows simulation $\mathrm{CALIF}^{3} \mathrm{~S}$ [5], developed at IRSN on the basis of the software components library PELICANS [55]; in this context, it is routinely used for industrial applications.

\subsubsection{The scheme}

In the algorithm given below, the velocity is predicted by solving the momentum balance equation with a known pressure. This latter is obtained from the beginning-of-step pressure through a "renormalization" step, in order to be able to perform the stability analysis (stability of the scheme and satisfaction of the entropy condition). Note that the renormalization proposed here is different than that proposed in [22] in the context of variable density incompressible flows or in [14] in the context of compressible barotropic flows. Indeed, in these latter works, this step requires the resolution of a discrete elliptic problem for the pressure, while, here, we only scale the pressure gradient by a simple weight. Then, the velocity is corrected and the other variables are advanced in time. As for the implicit scheme, a discrete kinetic energy balance can be derived, provided that the mass balance over the dual cells (3.5) holds; since the mass balance is not yet solved when performing the prediction step, this leads us to perform a time shift of the density at this stage. The algorithm reads, for $0 \leq n \leq N-1$ :

\section{Pressure gradient renormalization step:}

$$
\forall \sigma \in \mathcal{E}, \quad(\widetilde{\nabla p})_{\sigma}^{n+1}=\sqrt{\frac{\rho_{D_{\sigma}}^{n}}{\rho_{D_{\sigma}}^{n-1}}}(\nabla p)_{\sigma}^{n} .
$$

Prediction step - Solve for $\tilde{\boldsymbol{u}}^{n+1}$ :

For $1 \leq i \leq d, \forall \sigma \in \mathcal{E}_{\mathcal{S}}^{(i)}$,

$$
\frac{\left|D_{\sigma}\right|}{\delta t}\left(\rho_{D_{\sigma}}^{n} \tilde{u}_{\sigma, i}^{n+1}-\rho_{D_{\sigma}}^{n-1} u_{\sigma, i}^{n}\right)+\sum_{\epsilon \in \overline{\mathcal{E}}\left(D_{\sigma}\right)} F_{\sigma, \epsilon}^{n} \tilde{u}_{\epsilon, i}^{n+1}-\left|D_{\sigma}\right|\left(\Delta^{\mathcal{M}} \tilde{\boldsymbol{u}}\right)_{\sigma, i}^{n+1}+\left|D_{\sigma}\right|(\widetilde{\nabla p})_{\sigma, i}^{n+1}=0 .
$$


Correction step - Solve for $\rho^{n+1}, p^{n+1}$ and $\boldsymbol{u}^{n+1}$ :

For $1 \leq i \leq d, \forall \sigma \in \mathcal{E}_{\mathcal{S}}^{(i)}$

$$
\begin{gathered}
\frac{\left|D_{\sigma}\right|}{\delta t} \rho_{D_{\sigma}}^{n}\left(u_{\sigma, i}^{n+1}-\tilde{u}_{\sigma, i}^{n+1}\right)+\left|D_{\sigma}\right|\left[(\nabla p)_{\sigma, i}^{n+1}-(\widetilde{\nabla p})_{\sigma, i}^{n+1}\right]=0, \\
\forall K \in \mathcal{M}, \quad \frac{|K|}{\delta t}\left(\rho_{K}^{n+1}-\rho_{K}^{n}\right)+\sum_{\sigma \in \mathcal{E}(K)} F_{K, \sigma}^{n+1}=0, \text { with } F_{K, \sigma}^{n+1}=|\sigma| \rho_{\sigma}^{n+1} u_{K, \sigma}^{n+1}, \\
\forall K \in \mathcal{M}, \quad p_{K}^{n+1}=\left(\rho_{K}^{n+1}\right)^{\gamma} .
\end{gathered}
$$

Recall that the notation $\rho_{\sigma}^{n+1}$ in $(3.42 \mathrm{~d})$ stands for the upwind choice of $\rho$ defined by (3.3), while $\rho_{D_{\sigma}}^{n}$ (resp. $\left.\rho_{D_{\sigma}}^{n-1}\right)$ in $(3.42 \mathrm{~b})$ is the convex combination of $\rho_{K}^{n}$ and $\rho_{L}^{n}$ (resp. $\rho_{K}^{n-1}$ and $\rho_{L}^{n-1}$ ) defined by (3.4). The initialization of the scheme is performed as follows. First, $\rho^{-1}$ and $\boldsymbol{u}^{0}$ are given by the average of the initial conditions $\rho_{0}$ and $\boldsymbol{u}_{0}$ on the primal and dual cells respectively:

$$
\begin{aligned}
& \forall K \in \mathcal{M}, \quad \rho_{K}^{-1}=\frac{1}{|K|} \int_{K} \rho_{0}(\boldsymbol{x}) \mathrm{d} \boldsymbol{x}, \\
& \text { for } 1 \leq i \leq d, \forall \sigma \in \mathcal{E}_{\mathcal{S}}^{(i)}, \quad u_{\sigma, i}^{0}=\frac{1}{\left|D_{\sigma}\right|} \int_{D_{\sigma}}\left(\boldsymbol{u}_{0}(\boldsymbol{x})\right)_{i} \mathrm{~d} \boldsymbol{x} .
\end{aligned}
$$

Then, we compute $\rho^{0}$ by solving the mass balance equation (3.42d). Finally, the initial pressure $p^{0}$ is computed from the initial density $\rho^{0}$ by the equation of state: $\forall K \in \mathcal{M}, p_{K}^{0}=\left(\rho_{K}^{0}\right)^{\gamma}$. This procedure allows to perform the first prediction step with $\left(\rho_{D_{\sigma}}^{-1}\right)_{\sigma \in \mathcal{E}},\left(\rho_{D_{\sigma}}^{0}\right)_{\sigma \in \mathcal{E}}$ and the dual mass fluxes satisfying the mass balance.

\subsubsection{Estimates}

As for the implicit scheme, we begin with an estimate on the velocity which is a discrete equivalent of the kinetic energy balance.

Lemma 3.11 (Discrete kinetic energy balance, pressure correction scheme). A solution to the system (3.42) satisfies the following equality, for $1 \leq i \leq d, \sigma \in \mathcal{E}_{\mathcal{S}}^{(i)}$ and $0 \leq n \leq N-1$ :

$$
\frac{1}{2} \frac{\left|D_{\sigma}\right|}{\delta t}\left[\rho_{D_{\sigma}}^{n}\left(u_{\sigma, i}^{n+1}\right)^{2}-\rho_{D_{\sigma}}^{n-1}\left(u_{\sigma, i}^{n}\right)^{2}\right]+\frac{1}{2} \sum_{\epsilon=D_{\sigma} \mid D_{\sigma^{\prime}}} F_{\sigma, \epsilon}^{n} \tilde{u}_{\sigma, i}^{n+1} \tilde{u}_{\sigma^{\prime}, i}^{n+1}+\left|D_{\sigma}\right|(\nabla p)_{\sigma, i}^{n+1} u_{\sigma, i}^{n+1}=-R_{\sigma, i}^{n+1}-P_{\sigma, i}^{n+1},
$$

where

$$
\begin{aligned}
& R_{\sigma, i}^{n+1}=\frac{\left|D_{\sigma}\right|}{2 \delta t} \rho_{D_{\sigma}}^{n-1}\left(\tilde{u}_{\sigma, i}^{n+1}-u_{\sigma, i}^{n}\right)^{2}+\left[\sum_{\epsilon=D_{\sigma} \mid D_{\sigma^{\prime}}} \nu h_{\epsilon}^{d-2}\left(\tilde{u}_{\sigma, i}^{n+1}-\tilde{u}_{\sigma^{\prime}, i}^{n+1}\right)\right] \tilde{u}_{\sigma, i}^{n+1}, \\
& P_{\sigma, i}^{n+1}=\frac{\left|D_{\sigma}\right| \delta t}{2 \rho_{D_{\sigma}}^{n}}\left[\left((\nabla p)_{\sigma, i}^{n+1}\right)^{2}-\left((\widetilde{\nabla p})_{\sigma, i}^{n+1}\right)^{2}\right] .
\end{aligned}
$$

Proof. Let us multiply the velocity prediction equation (3.42b) by the corresponding velocity unknown $\tilde{u}_{\sigma, i}^{n+1}$, and use the equality (A.9) of Lemma A.2, on the dual mesh and with $P=D_{\sigma}$. We obtain:

$$
\begin{aligned}
\frac{1}{2} \frac{\left|D_{\sigma}\right|}{\delta t}\left[\rho_{D_{\sigma}}^{n}\left(\tilde{u}_{\sigma, i}^{n+1}\right)^{2}-\right. & \left.\rho_{D_{\sigma}}^{n-1}\left(u_{\sigma, i}^{n}\right)^{2}\right]+\frac{1}{2} \sum_{\epsilon=D_{\sigma} \mid D_{\sigma^{\prime}}} F_{\sigma, \epsilon}^{n} \tilde{u}_{\sigma, i}^{n+1} \tilde{u}_{\sigma^{\prime}, i}^{n+1} \\
& +\frac{1}{2} \frac{\left|D_{\sigma}\right|}{\delta t} \rho_{D_{\sigma}}^{n-1}\left(\tilde{u}_{\sigma, i}^{n+1}-u_{\sigma, i}^{n}\right)^{2}-\left|D_{\sigma}\right|\left(\Delta^{\mathcal{M}} \tilde{\boldsymbol{u}}\right)_{\sigma, i}^{n+1} \tilde{u}_{\sigma, i}^{n+1}+\left|D_{\sigma}\right|(\widetilde{\nabla})_{\sigma, i}^{n+1} \tilde{u}_{\sigma, i}^{n+1}=0 .
\end{aligned}
$$


Dividing the velocity correction equation $(3.42 \mathrm{c})$ by $\left(\frac{\left|D_{\sigma}\right|}{\delta t} \rho_{D_{\sigma}}^{n}\right)^{\frac{1}{2}}$, we obtain:

$$
\left[\frac{\left|D_{\sigma}\right|}{\delta t} \rho_{D_{\sigma}}^{n}\right]^{1 / 2} u_{\sigma, i}^{n+1}+\left[\frac{\left|D_{\sigma}\right| \delta t}{\rho_{D_{\sigma}}^{n}}\right]^{1 / 2}(\nabla p)_{\sigma, i}^{n+1}=\left[\frac{\left|D_{\sigma}\right|}{\delta t} \rho_{D_{\sigma}}^{n}\right]^{1 / 2} \tilde{u}_{\sigma, i}^{n+1}+\left[\frac{\left|D_{\sigma}\right| \delta t}{\rho_{D_{\sigma}}^{n}}\right]^{1 / 2}(\widetilde{\nabla p})_{\sigma, i}^{n+1} .
$$

Squaring this relation, dividing by two and summing it with (3.46) yields the result, using the definition (3.7) of $\left(\Delta^{\mathcal{M}} \tilde{\boldsymbol{u}}\right)^{n+1}$.

The discrete potential balance is again valid for the pressure correction algorithm, thanks to the fact that the mass balance (3.42d) is satisfied. The proof is identical to that of Lemma 3.2 given for the implicit scheme.

Lemma 3.12 (Discrete potential balance, pressure correction scheme). A solution to the system (3.42) satisfies the discrete potential balance (3.19), with $R_{K}^{n+1}$ defined by (3.20).

Let us now turn to the entropy inequality.

Proposition 3.13 (Global discrete entropy inequality, existence of a solution). Assume that the initial condition $\rho_{0}$ is positive. Then there exists a solution $\left(\boldsymbol{u}^{n}, \rho^{n}\right)_{0 \leq n \leq N}$ and $\left(\tilde{\boldsymbol{u}}^{n}\right)_{1 \leq n \leq N}$ to the scheme (3.42), and, for $1 \leq n \leq N, \rho^{n}>0$ and the following inequality holds:

$$
\frac{1}{2} \sum_{i=1}^{d} \sum_{\sigma \in \mathcal{E}_{\mathcal{S}}^{(i)}}\left|D_{\sigma}\right| \rho_{D_{\sigma}}^{n-1}\left(u_{\sigma, i}^{n}\right)^{2}+\sum_{K \in \mathcal{M}}|K| \mathcal{H}\left(\rho_{K}^{n}\right)+\mathcal{R}^{n} \leq C_{0}
$$

where $C_{0} \in \mathbb{R}_{+}$only depends on the initial conditions and on the density field $\rho^{0}$ computed at the initialization of the algorithm. The remainder term $\mathcal{R}^{n}$ is non-negative, and gathers some estimates of the space and time translates of the unknowns:

$$
\begin{aligned}
\mathcal{R}^{n}=\sum_{i=1}^{d} \sum_{k=1}^{n}[ & \left.\frac{1}{2} \sum_{\sigma \in \mathcal{E}_{\mathcal{S}}^{(i)}}\left|D_{\sigma}\right| \rho_{D_{\sigma}}^{k-2}\left(\tilde{u}_{\sigma, i}^{k}-u_{\sigma, i}^{k-1}\right)^{2}+\delta t \sum_{\epsilon=D_{\sigma} \mid D_{\sigma^{\prime}} \in \overline{\mathcal{E}}_{\mathcal{S}}^{(i)}} \nu h_{\sigma}^{d-2}\left(\tilde{u}_{\sigma, i}^{k}-\tilde{u}_{\sigma^{\prime}, i}^{k}\right)^{2}\right] \\
& +\frac{\gamma}{2} \sum_{k=1}^{n} \delta t \sum_{\sigma=K \mid L \in \mathcal{E}_{\mathrm{int}}}|\sigma|\left(\rho_{\sigma, \gamma}^{k}\right)^{\gamma-2}\left|u_{K, \sigma}^{k}\right|\left(\rho_{K}^{k}-\rho_{L}^{k}\right)^{2}+\frac{\delta t^{2}}{2} \sum_{\sigma \in \mathcal{E}_{\mathrm{int}}} \frac{\left|D_{\sigma}\right|}{\rho_{D_{\sigma}}^{n-1}}\left|(\nabla p)_{\sigma}^{n}\right|^{2},
\end{aligned}
$$

with $\rho_{\sigma, \gamma}^{k}$ equal to either $\rho_{K}^{k}$ or $\rho_{L}^{k}$ and such that $\left(\rho_{\sigma, \gamma}^{k}\right)^{\gamma-2}=\min \left(\left(\rho_{K}^{k}\right)^{\gamma-2},\left(\rho_{L}^{k}\right)^{\gamma-2}\right)$.

Proof. The essential arguments of the proof of this proposition are given in ([14], Thm. 3.8) with slightly different notations, so we briefly recall here how to obtain the estimate (3.47), for the sake of completeness. We sum the kinetic energy balance equation (3.44) over the faces, and the elastic potential balance (3.19) over the cells, and finally sum the two obtained relations. We obtain a "local in time" version of equation (3.47), which reads:

$$
\mathcal{T}^{n+1}-\mathcal{T}^{n}+\mathcal{R}^{n+1}+\mathcal{P}^{n+1}=0,
$$

where:

$$
\mathcal{T}^{n+1}=\sum_{K \in \mathcal{M}}|K| \mathcal{H}\left(\rho_{K}^{n+1}\right)+\frac{1}{2} \sum_{i=1}^{d} \sum_{\sigma \in \mathcal{E}_{\mathcal{S}}^{(i)}}\left|D_{\sigma}\right| \rho_{D_{\sigma}}^{n}\left(u_{\sigma, i}^{n+1}\right)^{2}
$$

and:

$$
\mathcal{R}^{n+1}=\sum_{1 \leq i \leq d} \sum_{\sigma \in \mathcal{E}_{\mathcal{S}}^{(i)}} R_{\sigma, i}^{n+1}, \quad \mathcal{P}^{n+1}=\sum_{1 \leq i \leq d} \sum_{\sigma \in \mathcal{E}_{\mathcal{S}}^{(i)} \cap \mathcal{E}_{\mathrm{int}}} P_{\sigma, i}^{n+1}
$$


with $R_{\sigma, i}^{n+1}$, and $P_{\sigma, i}^{n+1}$ given by equation (3.45). The term $\mathcal{P}^{n+1}$ thus reads:

$$
\mathcal{P}^{n+1}=\frac{\delta t^{2}}{2} \sum_{\sigma \in \mathcal{E}_{\text {int }}} \frac{\left|D_{\sigma}\right|}{\rho_{D_{\sigma}}^{n}}\left[\left|(\boldsymbol{\nabla} p)_{\sigma}^{n+1}\right|^{2}-\left|(\widetilde{\nabla} p)_{\sigma}^{n+1}\right|^{2}\right]=\frac{\delta t^{2}}{2} \sum_{\sigma \in \mathcal{E}_{\text {int }}}\left|D_{\sigma}\right|\left[\frac{\left|(\boldsymbol{\nabla} p)_{\sigma}^{n+1}\right|^{2}}{\rho_{D_{\sigma}}^{n}}-\frac{\left|(\boldsymbol{\nabla} p)_{\sigma}^{n}\right|^{2}}{\rho_{D_{\sigma}}^{n-1}}\right] .
$$

Then, summing (3.49) over the time steps yields the estimate (3.47) with:

$$
C_{0}=\sum_{K \in \mathcal{M}}|K| \mathcal{H}\left(\rho_{K}^{0}\right)+\frac{1}{2} \sum_{1 \leq i \leq d} \sum_{\sigma \in \mathcal{E}_{\mathcal{S}}^{(i)}}\left|D_{\sigma}\right| \rho_{D_{\sigma}}^{-1}\left(\boldsymbol{u}_{\sigma, i}^{0}\right)^{2}+\frac{\delta t^{2}}{2} \sum_{\sigma \in \mathcal{E}_{\mathrm{int}}} \frac{\left|D_{\sigma}\right|}{\rho_{D_{\sigma}}^{-1}}\left|(\boldsymbol{\nabla} p)_{\sigma}^{0}\right|^{2} .
$$

Remark 3.14. As in the implicit case, the inequality (3.47) thus provides an estimate on the unknowns (see Rem. 3.4).

Remark 3.15 (Regularity assumptions for the initial conditions). For a given mesh, the quantity denoted above by $C_{0}$ is bounded whenever $\rho_{0}$ is positive and belongs to $\mathrm{L}^{1}(\Omega)$ and $\boldsymbol{u}_{0}$ belongs to $\mathrm{L}^{1}(\Omega)^{d}$. When dealing with a sequence of discretizations to pass to the limit in the scheme, we need to assume that $C_{0}$ is controlled independently of the mesh and time step, which necessitates (i) that the initial kinetic energy is bounded, (ii) that $\mathcal{H}\left(\rho_{K}^{0}\right)$ is bounded in $\mathrm{L}^{1}(\Omega)$, and (iii) that the last term involving the discrete pressure gradient does not blow-up.

Assumption (ii) (and, of course, (i)) may be obtained by supposing that both $\boldsymbol{u}_{0}$ and $\rho_{0}$ belongs to $\mathrm{L}^{\infty}(\Omega)$ and $\mathrm{L}^{\infty}(\Omega)^{d}$ respectively and that $\delta t / h$ is bounded (possibly by a number much larger than 1 ); indeed, $\rho^{0}$ is then obtained in this case by a single time step of a (discrete) transport equation with a velocity field the divergence of which is controlled by $1 / h$, and so $\rho^{0}$ is controlled in $\mathrm{L}^{\infty}(\Omega)$. Assumption (iii) may then be inferred by the same assumption on the ratio $\delta t / h$, together with the hypothesis that the data $\rho_{0}$ (and so $\rho^{-1}$ ) is bounded away from zero. Indeed, since $\rho^{0}$ is bounded, so is $p^{0}$ and we get:

$$
\sum_{\sigma \in \mathcal{E}_{\mathrm{int}}} \frac{\left|D_{\sigma}\right| \delta t^{2}}{\rho_{D_{\sigma}}^{-1}}\left|(\nabla p)_{\sigma}^{0}\right|^{2}=\sum_{\sigma=K \mid L \in \mathcal{E}_{\mathrm{int}}} \frac{\delta t^{2}|\sigma|^{2}}{\rho_{D_{\sigma}}^{-1}\left|D_{\sigma}\right|}\left(p_{K}^{0}-p_{L}^{0}\right)^{2} \leq C\left\|\frac{1}{\rho^{-1}}\right\|_{L^{\infty}(\Omega)}\left\|p^{0}\right\|_{L^{\infty}(\Omega)}^{2} \sum_{\sigma \in \mathcal{E}_{\mathrm{int}}} \frac{h^{2}|\sigma|^{2}}{\left|D_{\sigma}\right|}
$$

and the last sum is bounded. We shall work under these assumptions for the passage to the limit in the scheme.

The expression (3.50) in the proof of Proposition 3.13, where $\mathcal{P}^{n+1}$ is set under the form of a difference of the same two quantities taken at two consecutive time steps, shows the interest of the pressure gradient renormalization step. We prove in addition in the following lemma that, under stability conditions, this remainder term tends to zero in a discrete distribution sense.

Lemma 3.16 (Pressure remainder terms). Let $\left(\mathcal{M}^{(m)}, \delta t^{(m)}\right)_{m \in \mathbb{N}}$ be a sequence of meshes and time steps, such that $h^{(m)}$ and $\delta t^{(m)}$ tend to zero as $m$ tends to infinity, and satisfying the CFL-like condition:

$$
\forall m \in \mathbb{N}, \quad \frac{\delta t^{(m)}}{\underline{h}^{(m)}} \leq C, \quad \text { with } \underline{h}^{(m)}=\min _{\sigma \in \mathcal{E}_{\mathrm{int}}^{(m)}} \frac{\left|D_{\sigma}\right|}{|\sigma|},
$$

and where $C$ is a positive real number which can be greater than 1. Let $\left(\rho^{(m)}\right)_{m \in \mathbb{N}}$ and $\left(p^{(m)}\right)_{m \in \mathbb{N}}$ be (part of) the associated sequence of discrete solutions, satisfying equations (3.42). We assume that the sequence $\left(p^{(m)}\right)_{m \in \mathbb{N}}$ is uniformly bounded in $\mathrm{L}^{\infty}(\Omega \times(0, T))$ and in the discrete $\mathrm{L}^{1}(0, T ; \mathrm{B} V(\Omega))$ norm:

$$
\forall m \in \mathbb{N}, \quad\left\|p^{(m)}\right\|_{\mathcal{T}, x, \mathrm{~B} V}=\sum_{n=0}^{N^{(m)}} \delta t \sum_{\sigma=K \mid L \in \mathcal{E}_{\text {int }}^{(m)}}|\sigma|\left|\left(p^{(m)}\right)_{L}^{n}-\left(p^{(m)}\right)_{K}^{n}\right| \leq C
$$


We furthermore suppose that $\left(\rho^{(m)}\right)_{m \in \mathbb{N}}$ and $\left(1 / \rho^{(m)}\right)_{m \in \mathbb{N}}$ are bounded in $\mathrm{L}^{\infty}(\Omega \times(0, T))$. Let $\varphi \in \mathrm{C}_{c}^{\infty}(\Omega \times[0, T))$, and, for $m \in \mathbb{N}, 0 \leq n \leq N^{(m)}$ and $\sigma \in \mathcal{E}_{\text {int }}^{(m)}$, let $\varphi_{\sigma}^{n}=\varphi\left(\boldsymbol{x}_{\sigma}, t^{n}\right)$, with $\boldsymbol{x}_{\sigma}$ the mass center of $\sigma$. Let us define the quantity $P_{\sigma}^{n+1}$, for $0 \leq n \leq N-1$ and $\sigma \in \mathcal{E}_{\text {int }}$, as:

$$
P_{\sigma}^{n+1}=\frac{\left|D_{\sigma}\right| \delta t}{\rho_{D_{\sigma}}^{n}}\left[\left|(\nabla p)_{\sigma}^{n+1}\right|^{2}-\left|(\widetilde{\nabla} p)_{\sigma}^{n+1}\right|^{2}\right] .
$$

Then

$$
\lim _{m \rightarrow+\infty}\left[\sum_{n=0}^{n-1} \delta t \sum_{\sigma \in \mathcal{E}_{\text {int }}} P_{\sigma}^{n+1} \varphi_{\sigma}^{n}\right]=0 .
$$

Proof. By definition of $P_{\sigma}^{n+1}$, we get that

$$
\sum_{n=0}^{N-1} \delta t \sum_{\sigma=K \mid L \in \mathcal{E}_{\mathrm{int}}} P_{\sigma}^{n+1} \varphi_{\sigma}^{n}=\sum_{n=0}^{N-1} \delta t^{2} \sum_{\sigma=K \mid L \in \mathcal{E}_{\mathrm{int}}} \frac{|\sigma|^{2}}{\left|D_{\sigma}\right|}\left[\frac{1}{\rho_{D_{\sigma}}^{n}}\left(p_{K}^{n+1}-p_{L}^{n+1}\right)^{2}-\frac{1}{\rho_{D_{\sigma}}^{n-1}}\left(p_{K}^{n}-p_{L}^{n}\right)^{2}\right] \varphi_{\sigma}^{n} .
$$

A discrete integration by parts yields:

$$
\begin{aligned}
\left|\sum_{n=0}^{N-1} \delta t \sum_{\sigma=K \mid L \in \mathcal{E}_{\mathrm{int}}} P_{\sigma}^{n+1} \varphi_{\sigma}^{n}\right| & \leq \delta t^{2}\left[\sum_{\sigma=K \mid L \in \mathcal{E}_{\mathrm{int}}} \frac{1}{\rho_{D_{\sigma}}^{-1}} \frac{|\sigma|^{2}}{\left|D_{\sigma}\right|}\left|p_{K}^{0}-p_{L}^{0}\right|^{2}\left|\varphi_{\sigma}^{0}\right|\right. \\
& \left.+\sum_{n=0}^{N-1} \sum_{\sigma=K \mid L \in \mathcal{E}_{\mathrm{int}}} \frac{1}{\rho_{D_{\sigma}}^{n}} \frac{|\sigma|^{2}}{\left|D_{\sigma}\right|}\left(p_{K}^{n+1}-p_{L}^{n+1}\right)^{2}\left|\varphi_{\sigma}^{n+1}-\varphi_{\sigma}^{n}\right|\right] .
\end{aligned}
$$

Using the fact that, for $0 \leq n \leq N$ and $\sigma=K\left|L \in \mathcal{E}_{\text {int }},\left(p_{K}^{n}-p_{L}^{n}\right)^{2} \leq 2\|p\|_{L^{\infty}(\Omega \times(0, T))}\right| p_{K}^{n}-p_{L}^{n} \mid$, we get, thanks to the regularity of $\varphi$, that

$$
\left|\sum_{n=0}^{N-1} \delta t \sum_{\sigma=K \mid L \in \mathcal{E}_{\mathrm{int}}} P_{\sigma}^{n+1} \varphi_{\sigma}^{n}\right| \leq C_{\varphi} \frac{\delta t^{2}}{\underline{\mathrm{h}}}\|p\|_{\mathrm{L}^{\infty}(\Omega \times(0, T))}\left[\left\|p^{0}\right\|_{\mathrm{B} V(\Omega)}+\|p\|_{\mathcal{T}, x, \mathrm{~B} V}\right],
$$

which concludes the proof.

\subsubsection{Passing to the limit in the scheme}

Using the notations of Section 3.1.3, the pressure correction scheme in one space dimension reads:

Initialization - Compute $\rho^{-1}, u^{0}$, solve for $\rho^{0}$ and compute $p^{0}$ :

$$
\begin{array}{ll}
\forall K \in \mathcal{M}, & \rho_{K}^{-1}=\frac{1}{|K|} \int_{K} \rho_{0}(x) \mathrm{d} x, \\
\forall \sigma \in \mathcal{E}_{\text {int }}, & u_{\sigma}^{0}=\frac{1}{\left|D_{\sigma}\right|} \int_{D_{\sigma}} u_{0}(x) \mathrm{d} x, \\
\forall K=\left[\overrightarrow{\sigma \sigma^{\prime}}\right] \in \mathcal{M}, & \frac{|K|}{\delta t}\left(\rho_{K}^{0}-\rho_{K}^{-1}\right)+F_{\sigma^{\prime}}^{0}-F_{\sigma}^{0}=0, \\
\forall K \in \mathcal{M}, & p_{K}^{0}=\left(\rho_{K}^{0}\right)^{\gamma} .
\end{array}
$$

\section{Pressure gradient renormalization step:}

$$
\forall \sigma=\overrightarrow{K \mid L} \in \mathcal{E}_{\mathrm{int}}, \quad(\widetilde{\delta p})_{\sigma}^{n+1}=\sqrt{\frac{\rho_{D_{\sigma}}^{n}}{\rho_{D_{\sigma}}^{n-1}}}\left(p_{L}^{n}-p_{K}^{n}\right) .
$$


Prediction step - Solve for $\tilde{u}^{n+1}$ :

$$
\begin{aligned}
& \forall \sigma=\overrightarrow{K \mid L} \in \mathcal{E}_{\text {int }}, \\
& \qquad \begin{array}{r}
\frac{\left|D_{\sigma}\right|}{\delta t}\left(\rho_{D_{\sigma}}^{n} \tilde{u}_{\sigma}^{n+1}-\rho_{D_{\sigma}}^{n-1} u_{\sigma}^{n}\right)+F_{L}^{n} \tilde{u}_{L}^{n+1}-F_{K}^{n} \tilde{u}_{K}^{n+1} \\
-\left|D_{\sigma}\right|\left(\Delta_{\mathcal{M}} \tilde{u}\right)_{\sigma}^{n+1}+(\widetilde{\delta p})_{\sigma}^{n+1}=0,
\end{array}
\end{aligned}
$$

Correction step - Solve for $\rho^{n+1}, p^{n+1}$ and $u^{n+1}$ :

$$
\begin{aligned}
& \forall \sigma=\overrightarrow{K \mid L} \in \mathcal{E}_{\text {int }}, \\
& \frac{\left|D_{\sigma}\right|}{\delta t} \rho_{D_{\sigma}}^{n}\left(u_{\sigma}^{n+1}-\tilde{u}_{\sigma}^{n+1}\right)+\left(p_{L}^{n+1}-p_{K}^{n+1}\right)-(\widetilde{\delta p})_{\sigma}^{n+1}=0, \\
& \forall K=\left[\overrightarrow{\sigma \sigma^{\prime}}\right] \in \mathcal{M}, \\
& \frac{|K|}{\delta t}\left(\rho_{K}^{n+1}-\rho_{K}^{n}\right)+F_{\sigma^{\prime}}^{n+1}-F_{\sigma}^{n+1}=0, \\
& \forall K \in \mathcal{M}, \quad p_{K}^{n+1}=\left(\rho_{K}^{n+1}\right)^{\gamma} .
\end{aligned}
$$

Theorem 3.17 (Consistency of the pressure correction scheme). Let $\Omega$ be an open bounded interval of $\mathbb{R}$. We suppose that $\rho_{0} \in \mathrm{L}^{\infty}(\Omega), 1 / \rho_{0} \in \mathrm{L}^{\infty}(\Omega)$ and $u_{0} \in \mathrm{L}^{\infty}(\Omega)$. Let $\left(\mathcal{M}^{(m)}, \delta t^{(m)}, \nu^{(m)}\right)_{m \in \mathbb{N}}$ be a regular sequence of discretizations in the sense of Definition 3.5, and let $\left(\rho^{(m)}, p^{(m)}, u^{(m)}, \tilde{u}^{(m)}\right)_{m \in \mathbb{N}}$ be the corresponding sequence of solutions. We suppose that this sequence converges in $\mathrm{L}^{p}(\Omega \times(0, T))^{4}$, for $1 \leq p<+\infty$, to $(\bar{\rho}, \bar{p}, \bar{u}, \overline{\tilde{u}}) \in$ $\mathrm{L}^{\infty}(\Omega \times(0, T))^{4}$. We suppose in addition that both sequences $\left(\rho^{(m)}\right)_{m \in \mathbb{N}}$ and $\left(1 / \rho^{(m)}\right)_{m \in \mathbb{N}}$ are uniformly bounded in $\mathrm{L}^{\infty}(\Omega \times(0, T))$.

Then $\bar{u}=\overline{\tilde{u}}$ and the triplet $(\bar{\rho}, \bar{p}, \bar{u})$ satisfies the system (3.28).

Proof. Let $m \in \mathbb{N}$ be given. Dropping for short the superscript ${ }^{(m)}$, the estimate of Proposition 3.13 yields:

$$
\sum_{k=1}^{n} \delta t \sum_{\sigma \in \mathcal{E}_{\text {int }}}\left|D_{\sigma}\right| \rho_{D_{\sigma}}^{k-1}\left(\tilde{u}_{\sigma}^{k}-u_{\sigma}^{k-1}\right)^{2} \leq C \delta t,
$$

where, by the assumption on the initial data, the real number $C$ is independent of $m$ (see Rem. 3.15). Hence,

$$
\left\|\tilde{u}^{(m)}-u^{(m)}(., .-\delta t)\right\|_{L^{2}(\Omega \times(0, T))}^{2} \leq C \delta t^{(m)}\left\|\frac{1}{\rho^{(m)}}\right\|_{L^{\infty}(\Omega \times(0, T))} .
$$

Letting $m$ tend to $+\infty$ in this equation yields $\bar{u}=\overline{\tilde{u}}$.

As for the implicit scheme, with the assumed convergence for the sequence of solutions, the limit satisfies the equation of state. The passage to the limit in the mass balance equation is the same as in the implicit case, and we only need to address here the momentum balance equation. Let $\varphi \in C_{c}^{\infty}(\Omega \times[0, T)$ ), and let its interpolate $\varphi_{\mathcal{E}}$ and its discrete derivatives be defined by Definition 3.6. Summing the velocity prediction and correction equations, multiplying the result by $\delta t \varphi_{\sigma}^{n}$ and then summing over the faces and time steps, we get $T_{1}^{(m)}+T_{2}^{(m)}+T_{3}^{(m)}+T_{4}^{(m)}=0$, with:

$$
\begin{aligned}
T_{1}^{(m)} & =\sum_{n=0}^{N-1} \sum_{\sigma \in \mathcal{E}_{\mathrm{int}}}\left|D_{\sigma}\right|\left[\rho_{D_{\sigma}}^{n} \tilde{u}_{\sigma}^{n+1}-\rho_{D_{\sigma}}^{n-1} u_{\sigma}^{n}\right] \varphi_{\sigma}^{n}, \\
T_{2}^{(m)} & =\sum_{n=0}^{N-1} \delta t \sum_{\sigma=\overrightarrow{K \mid L} \in \mathcal{E}_{\mathrm{int}}}\left[F_{L}^{n} \tilde{u}_{L}^{n+1}-F_{K}^{n} \tilde{u}_{K}^{n+1}\right] \varphi_{\sigma}^{n},
\end{aligned}
$$




$$
\begin{aligned}
T_{3}^{(m)} & =\sum_{n=0}^{N-1} \delta t \sum_{\sigma=\widehat{K \mid L} \in \mathcal{E}_{\mathrm{int}}}\left(p_{L}^{n+1}-p_{K}^{n+1}\right) \varphi_{\sigma}^{n}, \\
T_{4}^{(m)} & =\sum_{n=0}^{N-1} \delta t \sum_{\sigma \in \mathcal{E}_{\text {int }}}\left[\sum_{K=\left[\sigma \sigma^{\prime}\right]} \frac{\nu}{h_{K}}\left(\tilde{u}_{\sigma}^{n+1}-\tilde{u}_{\sigma^{\prime}}^{n+1}\right)\right] \varphi_{\sigma}^{n} .
\end{aligned}
$$

Thanks to the definition (3.26) of the density on the dual mesh $\rho_{D_{\sigma}}$, reordering the sums, we get for $T_{1}^{(m)}$ :

$$
\begin{aligned}
T_{1}^{(m)} & =-\sum_{n=0}^{N-1} \delta t \sum_{\sigma=K \mid L \in \mathcal{E}_{\mathrm{int}}}\left[\frac{|K|}{2} \rho_{K}^{n}+\frac{|L|}{2} \rho_{L}^{n}\right] \tilde{u}_{\sigma}^{n+1} \frac{\varphi_{\sigma}^{n+1}-\varphi_{\sigma}^{n}}{\delta t}-\sum_{\sigma=K \mid L \in \mathcal{E}_{\mathrm{int}}}\left[\frac{|K|}{2} \rho_{K}^{-1}+\frac{|L|}{2} \rho_{L}^{-1}\right] u_{\sigma}^{0} \varphi_{\sigma}^{0} \\
& =-\int_{0}^{T} \int_{\Omega} \rho^{(m)} \tilde{u}^{(m)} \partial_{t} \varphi_{\mathcal{M}^{(m)}} \mathrm{d} x \mathrm{~d} t-\int_{\Omega}\left(\rho^{(m)}\right)^{-1}(x)\left(u^{(m)}\right)^{0}(x) \varphi_{\mathcal{M}^{(m)}}(x, 0) \mathrm{d} x .
\end{aligned}
$$

From the definition (3.53a) of the initial conditions, the sequences $\left(\left(\rho^{(m)}\right)^{-1}\right)$ and $\left.\left(u^{(m)}\right)^{0}\right)$ converge in $\mathrm{L}^{r}(\Omega)$, for $r \geq 1$, to $\rho_{0}$ and $u_{0}$ respectively. Thanks to the convergence assumption for the sequence of discrete solutions, and noting that the sequence $\left(\rho^{(m)}(\cdot, \cdot-\delta t)\right)_{m \in \mathbb{N}}$ converges to $\bar{\rho}$ as $\left(\rho^{(m)}\right)_{m \in \mathbb{N}}$, we get:

$$
\lim _{m \longrightarrow+\infty} T_{1}^{(m)}=-\int_{0}^{T} \int_{\Omega} \bar{\rho} \bar{u} \partial_{t} \varphi \mathrm{d} x \mathrm{~d} t-\int_{\Omega} \rho_{0}(x) u_{0}(x) \varphi(x, 0) \mathrm{d} x .
$$

Let us now turn to $T_{2}^{(m)}$. From the expression (3.26) of the fluxes $F_{K}$ and the values $u_{K}$, reordering the sums, we get:

$$
T_{2}^{(m)}=-\frac{1}{4} \sum_{n=0}^{N-1} \delta t \sum_{K=\left[\overrightarrow{\left.\sigma \sigma^{\prime}\right]}\right] \in \mathcal{M}}\left(\rho_{\sigma}^{n} u_{\sigma}^{n}+\rho_{\sigma^{\prime}}^{n} u_{\sigma^{\prime}}^{n}\right)\left(\tilde{u}_{\sigma}^{n+1}+\tilde{u}_{\sigma^{\prime}}^{n+1}\right)\left(\varphi_{\sigma^{\prime}}^{n}-\varphi_{\sigma}^{n}\right),
$$

which we write $T_{2}^{(m)}=\mathcal{T}_{2}^{(m)}+\mathcal{R}_{2}^{(m)}$ with:

$$
\begin{aligned}
\mathcal{T}_{2}^{(m)} & =-\frac{1}{2} \sum_{n=0}^{N-1} \delta t \sum_{K=\left[\overrightarrow{\left.\sigma \sigma^{\prime}\right]} \in \mathcal{M}\right.} \rho_{K}^{n}\left[u_{\sigma}^{n} \tilde{u}_{\sigma}^{n+1}+u_{\sigma^{\prime}}^{n} \tilde{u}_{\sigma^{\prime}}^{n+1}\right]\left(\varphi_{\sigma^{\prime}}^{n}-\varphi_{\sigma}^{n}\right) . \\
& =-\int_{0}^{T} \int_{\Omega} \rho^{(m)}(\cdot, \cdot-\delta t) u^{(m)}(\cdot, \cdot-\delta t) \tilde{u}^{(m)} \check{\partial}_{x} \varphi_{\mathcal{E}} \mathrm{d} x \mathrm{~d} t
\end{aligned}
$$

and therefore,

$$
\lim _{m \longrightarrow+\infty} \mathcal{T}_{2}^{(m)}=-\int_{0}^{T} \int_{\Omega} \bar{\rho} \bar{u}^{2} \partial_{x} \varphi \mathrm{d} x \mathrm{~d} t .
$$

The remainder term $\mathcal{R}_{2}^{(m)}$ reads:

$$
\begin{aligned}
\mathcal{R}_{2}^{(m)}= & -\frac{1}{4} \sum_{n=0}^{N-1} \delta t \sum_{K=\left[\overrightarrow{\sigma \sigma^{\prime}}\right] \in \mathcal{M}}\left[\left(\rho_{\sigma}^{n} u_{\sigma}^{n}+\rho_{\sigma^{\prime}}^{n} u_{\sigma^{\prime}}^{n}\right)\left(\tilde{u}_{\sigma}^{n+1}+\tilde{u}_{\sigma^{\prime}}^{n+1}\right)\right. \\
& \left.-2 \rho_{K}^{n}\left(u_{\sigma}^{n} \tilde{u}_{\sigma}^{n+1}+u_{\sigma^{\prime}}^{n} \tilde{u}_{\sigma^{\prime}}^{n+1}\right)\right]\left(\varphi_{\sigma^{\prime}}^{n}-\varphi_{\sigma}^{n}\right) .
\end{aligned}
$$


Expanding the quantity $2 \rho_{K}^{n}\left(u_{\sigma}^{n} \tilde{u}_{\sigma}^{n+1}+u_{\sigma^{\prime}}^{n} \tilde{u}_{\sigma^{\prime}}^{n+1}\right)$ thanks to the identity $2(a b+c d)=(a+c)(b+d)+(a-c)(b-d)$, we get $\mathcal{R}_{2}^{(m)}=\mathcal{R}_{2,1}^{(m)}+\mathcal{R}_{2,2}^{(m)}$ :

$$
\begin{aligned}
& \mathcal{R}_{2,1}^{(m)}=-\frac{1}{4} \sum_{n=0}^{N-1} \delta t \sum_{K=\left[\overrightarrow{\left.\sigma \sigma^{\prime}\right]}\right.}\left(\left(\rho_{\sigma}^{n}-\rho_{K}^{n}\right) u_{\sigma}^{n}+\left(\rho_{\sigma^{\prime}}^{n}-\rho_{K}^{n}\right) u_{\sigma^{\prime}}^{n}\right)\left(\tilde{u}_{\sigma}^{n+1}+\tilde{u}_{\sigma^{\prime}}^{n+1}\right)\left(\varphi_{\sigma^{\prime}}^{n}-\varphi_{\sigma}^{n}\right), \\
& \mathcal{R}_{2,2}^{(m)}=\frac{1}{4} \sum_{n=0}^{N-1} \delta t \sum_{K=\left[\overrightarrow{\sigma \sigma^{\prime}}\right] \in \mathcal{M}} \rho_{K}^{n}\left(u_{\sigma}^{n}-u_{\sigma^{\prime}}^{n}\right)\left(\tilde{u}_{\sigma}^{n+1}-\tilde{u}_{\sigma^{\prime}}^{n+1}\right)\left(\varphi_{\sigma^{\prime}}^{n}-\varphi_{\sigma}^{n}\right) .
\end{aligned}
$$

First we study $\mathcal{R}_{2,1}^{(m)}$. Thanks to the definition of the upwind approximation, reordering the sum by faces, we get:

$$
\mathcal{R}_{2,1}^{(m)}=\frac{1}{4} \sum_{n=0}^{N-1} \delta t \sum_{\substack{\sigma \in \mathcal{E}_{\text {int }}, \sigma=L \rightarrow K, K=\left[\sigma \sigma^{\prime}\right]}} \varepsilon_{\sigma}^{n}\left(\rho_{L}^{n}-\rho_{K}^{n}\right) u_{\sigma}^{n}\left(\tilde{u}_{\sigma}^{n+1}+\tilde{u}_{\sigma^{\prime}}^{n+1}\right)\left(\varphi_{\sigma}^{n}-\varphi_{\sigma^{\prime}}^{n}\right),
$$

where we recall that the notation $\sigma=L \rightarrow K$ means that the face $\sigma$ separates $K$ and $L$ and the flow goes from $L$ to $K$ at the time level $n$, and where $\varepsilon_{\sigma}^{n}= \pm 1$. Since $\left|\varphi_{\sigma}^{n}-\varphi_{\sigma^{\prime}}^{n}\right| \leq C_{\varphi}|K| \leq C_{\varphi}\left(\left|D_{\sigma}\right|+\left|D_{\sigma^{\prime}}\right|\right)$, we get:

$$
\left|\mathcal{R}_{2,1}^{(m)}\right| \leq \frac{C_{\varphi}}{4} \sum_{n=0}^{N-1} \delta t \sum_{\substack{\sigma \in \mathcal{E}_{\text {int }}, \sigma=L \rightarrow K, K=\left[\sigma \sigma^{\prime}\right]}}\left(\left|D_{\sigma}\right|+\left|D_{\sigma^{\prime}}\right|\right)\left|\rho_{L}^{n}-\rho_{K}^{n}\right|\left|u_{\sigma}^{n}\right|\left|\tilde{u}_{\sigma}^{n+1}+\tilde{u}_{\sigma^{\prime}}^{n+1}\right|
$$

Therefore, by the Cauchy-Schwarz inequality, we get:

$$
\begin{aligned}
\left|\mathcal{R}_{2,1}^{(m)}\right| & \leq \frac{C_{\varphi}}{4}\left(h^{(m)}\right)^{1 / 2}\left[\sum_{n=0}^{N-1} \delta t \sum_{\sigma=K \mid L \in \mathcal{E}_{\text {int }}}\left|u_{\sigma}^{n}\right|\left(\rho_{L}^{n}-\rho_{K}^{n}\right)^{2}\right]^{1 / 2} \\
& \times\left[\sum_{n=0}^{N-1} \delta t \sum_{\substack{\sigma \in \mathcal{E}_{\text {int }}, \sigma=L \rightarrow K, K=\left[\sigma \sigma^{\prime}\right]}}\left(\left|D_{\sigma}\right|+\left|D_{\sigma^{\prime}}\right|\right)\left|u_{\sigma}^{n}\right|\left(\tilde{u}_{\sigma}^{n+1}+\tilde{u}_{\sigma^{\prime}}^{n+1}\right)^{2}\right]^{1 / 2} .
\end{aligned}
$$

Since the ratio of the size of two neighboring meshes is bounded by the regularity assumption on the mesh, we get from the estimate (3.47) on the solution:

$$
\left|\mathcal{R}_{2,1}^{(m)}\right| \leq C\left(h^{(m)}\right)^{1 / 2}\left[\left\|u^{(m)}\right\|_{\mathrm{L}^{2}(\Omega \times(0, T))}+\left\|\tilde{u}^{(m)}\right\|_{\mathrm{L}^{4}(\Omega \times(0, T))}^{2}\right],
$$

where $C \in \mathbb{R}_{+}$does not depend on $m$, and so $\mathcal{R}_{2,1}^{(m)}$ tends to zero when $m$ tends to $+\infty$. For $\mathcal{R}_{2,2}^{(m)}$, we have, by the Cauchy-Schwarz inequality:

$$
\begin{aligned}
\left|\mathcal{R}_{2,2}^{(m)}\right| & \leq C_{\varphi} \sum_{n=0}^{N-1} \delta t \sum_{K=\left[\overline{\left.\sigma \sigma^{\prime}\right]} \in \mathcal{M}\right.}|K| \rho_{K}^{n+1}\left|u_{\sigma}^{n}+u_{\sigma^{\prime}}^{n}\right|\left(\tilde{u}_{\sigma}^{n+1}-\tilde{u}_{\sigma^{\prime}}^{n+1}\right) \\
& \leq C_{\varphi} \frac{h^{(m)}}{\left(\nu^{(m)}\right)^{1 / 2}}\left\|\rho^{(m)}\right\|_{L^{\infty}(\Omega \times(0, T))}\left\|u^{(m)}\right\|_{L^{2}(\Omega \times(0, T))}\left[\sum_{n=0}^{N-1} \delta t \sum_{K=\left[\sigma \sigma^{\prime}\right] \in \mathcal{M}} \frac{\nu^{(m)}}{h_{K}}\left(\tilde{u}_{\sigma}^{n+1}-\tilde{u}_{\sigma^{\prime}}^{n+1}\right)^{2}\right]^{1 / 2},
\end{aligned}
$$

and thus, thanks to the estimate (3.47):

$$
\left|\mathcal{R}_{2,2}^{(m)}\right| \leq C \frac{h^{(m)}}{\left(\nu^{(m)}\right)^{1 / 2}}\left\|\rho^{(m)}\right\|_{L^{\infty}(\Omega \times(0, T))}\left\|u^{(m)}\right\|_{L^{2}(\Omega \times(0, T))},
$$


where $C \in \mathbb{R}_{+}$does not depend on $m$. Therefore, this term also tends to zero when $m$ tends to $+\infty$.

Next, we turn to the term $T_{3}^{(m)}$ :

$$
T_{3}^{(m)}=-\sum_{n=0}^{N-1} \delta t \sum_{K=\left[\overrightarrow{\left.\sigma \sigma^{\prime}\right]} \in \mathcal{M}\right.}|K| p_{K}^{n+1} \frac{\varphi_{\sigma^{\prime}}^{n}-\varphi_{\sigma}^{n}}{h_{K}}=-\int_{0}^{T} \int_{\Omega} p^{(m)} \oiint_{x} \varphi_{\mathcal{E}} \mathrm{d} x \mathrm{~d} t
$$

and therefore,

$$
\lim _{m \longrightarrow+\infty} T_{3}^{(m)}=-\int_{0}^{T} \int_{\Omega} \bar{p} \partial_{x} \varphi \mathrm{d} x \mathrm{~d} t
$$

Let us finally study $T_{4}^{(m)}$. Reordering the sums, we get:

$$
T_{4}^{(m)}=\sum_{n=0}^{N-1} \delta t \sum_{K=\left[\overrightarrow{\left.\sigma \sigma^{\prime}\right]} \in \mathcal{M}\right.} \frac{\nu^{(m)}}{h_{K}}\left(\tilde{u}_{\sigma}^{n+1}-\tilde{u}_{\sigma^{\prime}}^{n+1}\right)\left(\varphi_{\sigma}^{n}-\varphi_{\sigma^{\prime}}^{n}\right)
$$

The Cauchy-Schwarz inequality yields:

$$
\left|T_{4}^{(m)}\right| \leq\left[\sum_{n=0}^{N-1} \delta t \sum_{K=\left[\overrightarrow{\left.\sigma \sigma^{\prime}\right]} \in \mathcal{M}\right.} \frac{\nu^{(m)}}{h_{K}}\left(\tilde{u}_{\sigma}^{n+1}-\tilde{u}_{\sigma^{\prime}}^{n+1}\right)^{2}\right]^{1 / 2}\left[\sum_{n=0}^{N-1} \delta t \sum_{K=\left[\overrightarrow{\sigma \sigma^{\prime}}\right] \in \mathcal{M}} \frac{\nu^{(m)}}{h_{K}}\left(\varphi_{\sigma}^{n}-\varphi_{\sigma^{\prime}}^{n}\right)^{2}\right]^{1 / 2}
$$

and thus, again in view of the estimate (3.47), this term tends to zero when $\nu^{(m)}$ tends to zero.

Remark 3.18 (On the "non appearance of void assumption"). The assumption that $\left(1 / \rho^{(m)}\right)_{m \in \mathbb{N}}$ is bounded in $\mathrm{L}^{\infty}(\Omega \times(0, T))$ (which, loosely speaking, means that the appearance of void is excluded) is used twice in the proof of Theorem 3.17. We use it for the first time to obtain $\bar{u}=\overline{\tilde{u}}$. Here, the hypothesis may be circumvented by replacing this conclusion by $\bar{\rho} \bar{u}=\bar{\rho} \overline{\tilde{u}}$ (or, in other words, $\bar{u}=\overline{\tilde{u}}$ wherever $\bar{\rho} \neq 0$ ), which is easily obtained from inequality (3.54) below. The second time is to obtain, as in the implicit case, the "non-weighted" estimate of the density space translates (3.32) for $\gamma \geq 2$, see Remark 3.8.

Remark 3.19 (Less sharp bounds and more general meshes). As in the implicit case, the assumption that the ratio of the size of two neighboring cells is bounded, i.e. Assumption (ii) of Definition 3.5, is only used for the remainder associated with the the convection term in the momentum balance. It may be avoided if we suppose that the sequence of solution is uniformly bounded, replacing (3.58) by

$$
\left|\mathcal{R}_{2,1}^{(m)}\right| \leq C\left(h^{(m)}\right)^{1 / 2}\left\|u^{(m)}\right\|_{L^{\infty}(\Omega \times(0, T))}^{1 / 2}\left\|\tilde{u}^{(m)}\right\|_{L^{\infty}(\Omega \times(0, T))} .
$$

We now turn to the entropy condition. For any piecewise constant function $q$ on primal cells, we define its $\mathrm{L}^{1}(0, T ; \mathrm{B} V(\Omega))$ norm by:

$$
\|q\|_{\mathcal{T}, x, \mathrm{~B} V}=\sum_{n=0}^{N} \delta t \sum_{\sigma=K \mid L \in \mathcal{E}_{\text {int }}}\left|q_{L}^{n}-q_{K}^{n}\right| .
$$

With this notation, we are now in position to state the following result. 
Theorem 3.20 (Entropy consistency, pressure correction scheme). Under the assumptions of Theorem 3.17, we furthermore assume that:

- the sequence of regular meshes satisfies:

$$
\forall m \in \mathbb{N}, \quad \frac{\delta t^{(m)}}{\underline{h}^{(m)}} \leq C, \quad \text { with } \underline{h}^{(m)}=\min _{\sigma \in \mathcal{E}_{\text {int }}^{(m)}}\left|h_{\sigma}\right|,
$$

and where $C$ is a positive real number which can be greater than 1 ,

- the sequence $\left(p^{(m)}\right)_{m \in \mathbb{N}}$ is uniformly bounded in the discrete $\mathrm{L}^{1}(0, T ; \mathrm{B} V(\Omega))$ norm defined by (3.59).

Then the limit $(\bar{\rho}, \bar{p}, \bar{u})$ satisfies the entropy condition (3.35).

Proof. Let $\varphi \in \mathrm{C}_{c}^{\infty}(\Omega \times[0, T)), \varphi \geq 0$. Again using the notations of Definition 3.6, we multiply the kinetic balance equation (3.44) by $\varphi_{\sigma}^{n}$, and the elastic potential balance (3.19) by $\varphi_{K}^{n}$, sum over the faces and cells respectively and over the time steps, to get:

$$
\sum_{\sigma \in \mathcal{E}_{\mathrm{int}}} T_{\sigma}^{n+1} \varphi_{\sigma}^{n}+\sum_{K \in \mathcal{M}} T_{K}^{n+1} \varphi_{K}^{n}=-\sum_{\sigma \in \mathcal{E}_{\mathrm{int}}} R_{\sigma}^{n+1} \varphi_{\sigma}^{n}-\sum_{K \in \mathcal{M}} R_{K}^{n+1} \varphi_{K}^{n}-\sum_{\sigma \in \mathcal{E}_{\mathrm{int}}} P_{\sigma}^{n+1} \varphi_{\sigma}^{n},
$$

where, for $\sigma=\overrightarrow{K \mid L}, K=\left[\overrightarrow{\sigma^{\prime} \sigma}\right]$ and $L=\left[\overrightarrow{\sigma \sigma^{\prime \prime}}\right]$,

$$
T_{\sigma}^{n+1}=\frac{1}{2} \frac{\left|D_{\sigma}\right|}{\delta t}\left[\rho_{D_{\sigma}}^{n}\left(u_{\sigma}^{n+1}\right)^{2}-\rho_{D_{\sigma}}^{n-1}\left(u_{\sigma}^{n}\right)^{2}\right]+\frac{1}{2} F_{L}^{n+1} \tilde{u}_{\sigma}^{n+1} \tilde{u}_{\sigma^{\prime \prime}}^{n+1}-\frac{1}{2} F_{K}^{n+1} \tilde{u}_{\sigma}^{n+1} \tilde{u}_{\sigma^{\prime}}^{n+1}+\left(p_{L}^{n+1}-p_{K}^{n+1}\right) u_{\sigma}^{n+1},
$$

for $K=\left[\overrightarrow{\sigma \sigma^{\prime}}\right]$

$$
T_{K}^{n+1}=\frac{|K|}{\delta t}\left[\mathcal{H}\left(\rho_{K}^{n+1}\right)-\mathcal{H}\left(\rho_{K}^{n}\right)\right]+u_{\sigma^{\prime}}^{n+1} \mathcal{H}\left(\rho_{\sigma^{\prime}}^{n+1}\right)-u_{\sigma}^{n+1} \mathcal{H}\left(\rho_{\sigma}^{n+1}\right)+p_{K}^{n+1}\left(u_{\sigma^{\prime}}^{n+1}-u_{\sigma}^{n+1}\right),
$$

the quantities $R_{\sigma}^{n+1}$ and $P_{\sigma}^{n+1}$ are given by (the one-dimensional version of) equation (3.45), and $R_{K}^{n+1}$ is given by (the one-dimensional version of) equation (3.20).

The discrete weak form of the entropy balance is obtained by integrating in time (i.e. summing over the time steps) equation (3.60). We obtain $T_{1}^{(m)}+T_{2}^{(m)}+T_{3}^{(m)}+T_{4}^{(m)}+T_{5}^{(m)}+R^{(m)}+P^{(m)}=0$ where $T_{1}^{(m)}$, $T_{2}^{(m)}, T_{4}^{(m)}, T_{5}^{(m)}$ and $R^{(m)}$ are identical to their namesakes in the implicit case (see proof of Thm. 3.10, defined by $(3.37 \mathrm{a}),(3.37 \mathrm{~b}),(3.37 \mathrm{~d}),(3.37 \mathrm{e})$ and $(3.37 \mathrm{f})$ respectively, and

$$
\begin{aligned}
T_{3}^{(m)} & =\frac{1}{2} \sum_{n=0}^{N-1} \delta t \sum_{\substack{\sigma=\overrightarrow{K \mid L} \in \mathcal{E}_{\text {int }}, K=\left[\overrightarrow{\sigma^{\prime} \sigma}\right], L=\left[\overrightarrow{\sigma \sigma^{\prime \prime}}\right]}}\left[F_{L}^{n+1} \tilde{u}_{\sigma}^{n+1} \tilde{u}_{\sigma^{\prime \prime}}^{n+1}-F_{K}^{n+1} \tilde{u}_{\sigma}^{n+1} \tilde{u}_{\sigma^{\prime}}^{n+1}\right] \varphi_{\sigma}^{n}, \\
P^{(m)} & =\sum_{n=0}^{N-1} \delta t \sum_{\sigma \in \mathcal{E}_{\text {int }}} P_{\sigma}^{n+1} \varphi_{\sigma}^{n} .
\end{aligned}
$$

The terms $T_{1}^{(m)}, T_{2}^{(m)}, T_{4}^{(m)}, T_{5}^{(m)}$ and $R^{(m)}$ were studied in the proof of Theorem 3.10. Let us then study the kinetic energy convection term $T_{3}^{(m)}$ which reads, after reordering the summations:

$$
T_{3}^{(m)}=-\frac{1}{2} \sum_{n=0}^{N-1} \delta t \sum_{K=\left[\overrightarrow{\sigma \sigma^{\prime}}\right] \in \mathcal{M}} F_{K}^{n} \tilde{u}_{\sigma}^{n+1} \tilde{u}_{\sigma^{\prime}}^{n+1}\left(\varphi_{\sigma^{\prime}}^{n}-\varphi_{\sigma}^{n}\right) .
$$


We write $T_{3}^{m}=\mathcal{T}_{3}^{(m)}+\mathcal{R}_{3}^{(m)}$, where

$$
\begin{aligned}
\mathcal{T}_{3}^{(m)} & =-\frac{1}{2} \sum_{n=0}^{N-1} \delta t \sum_{K=\left[\overrightarrow{\sigma \sigma^{\prime}}\right] \in \mathcal{M}} \frac{|K|}{2} \rho_{K}^{n}\left[u_{\sigma}^{n}\left(\tilde{u}_{\sigma}^{n+1}\right)^{2}+u_{\sigma^{\prime}}^{n}\left(\tilde{u}_{\sigma^{\prime}}^{n+1}\right)^{2}\right] \frac{\varphi_{\sigma^{\prime}}^{n}-\varphi_{\sigma}^{n}}{h_{K}} \\
& =-\frac{1}{2} \int_{0}^{T} \int_{\Omega} \rho^{(m)}(x, t-\delta t) u^{(m)}(x, t-\delta t)\left(\tilde{u}^{(m)}(x, t)\right)^{2} ð_{x} \varphi_{\mathcal{E}} \mathrm{d} x \mathrm{~d} t
\end{aligned}
$$

so that

$$
\lim _{m \longrightarrow+\infty} \mathcal{T}_{3}^{(m)}=-\frac{1}{2} \int_{0}^{T} \int_{\Omega} \bar{\rho} \bar{u}^{3} \partial_{x} \varphi \mathrm{d} x \mathrm{~d} t
$$

The remainder term $\mathcal{R}_{3}^{(m)}$ reads:

$$
\mathcal{R}_{3}^{(m)}=-\frac{1}{4} \sum_{n=0}^{N-1} \delta t \sum_{K=\left[\overrightarrow{\sigma \sigma^{\prime}}\right] \in \mathcal{M}}\left[\left(\rho_{\sigma}^{n} u_{\sigma}^{n}+\rho_{\sigma^{\prime}}^{n} u_{\sigma^{\prime}}^{n}\right) \tilde{u}_{\sigma^{\prime}}^{n+1} \tilde{u}_{\sigma}^{n+1}-\rho_{K}^{n}\left(u_{\sigma}^{n}\left(\tilde{u}_{\sigma}^{n+1}\right)^{2}+u_{\sigma^{\prime}}^{n}\left(\tilde{u}_{\sigma^{\prime}}^{n+1}\right)^{2}\right)\right]\left(\varphi_{\sigma^{\prime}}^{n}-\varphi_{\sigma}^{n}\right) .
$$

Reordering the terms in the sum, we get:

$$
\mathcal{R}_{3}^{(m)}=-\frac{1}{4} \sum_{n=0}^{N-1} \delta t \sum_{K=\left[\overrightarrow{\sigma \sigma^{\prime}}\right] \in \mathcal{M}}[\underbrace{\left(\rho_{\sigma}^{n} \tilde{u}_{\sigma^{\prime}}^{n+1}-\rho_{K}^{n} \tilde{u}_{\sigma}^{n+1}\right) u_{\sigma}^{n} \tilde{u}_{\sigma}^{n+1}}_{\mathcal{D}_{1}}+\underbrace{\left(\rho_{\sigma^{\prime}}^{n} \tilde{u}_{\sigma}^{n+1}-\rho_{K}^{n} \tilde{u}_{\sigma^{\prime}}^{n+1}\right) u_{\sigma^{\prime}}^{n} \tilde{u}_{\sigma^{\prime}}^{n+1}}_{\mathcal{D}_{2}}]\left(\varphi_{\sigma^{\prime}}^{n}-\varphi_{\sigma}^{n}\right) .
$$

Let us consider the term involving $\mathcal{D}_{1}$, and skip the exposition of the treatment of the term with $\mathcal{D}_{2}$, which is similar. Using the identity $2(a b-c d)=(a-c)(b+d)+(a+c)(b-d)$, we split this first part of $\mathcal{R}_{3}^{(m)}$ into $\mathcal{R}_{31}^{(m)}+\mathcal{R}_{32}^{(m)}$, with:

$$
\begin{aligned}
& \mathcal{R}_{31}^{(m)}=-\frac{1}{8} \sum_{n=0}^{N-1} \delta t \sum_{K=\left[\overrightarrow{\sigma \sigma^{\prime}}\right] \in \mathcal{M}} u_{\sigma}^{n} \tilde{u}_{\sigma}^{n+1}\left(\rho_{\sigma}^{n}-\rho_{K}^{n}\right)\left(\tilde{u}_{\sigma^{\prime}}^{n+1}+\tilde{u}_{\sigma}^{n+1}\right)\left(\varphi_{\sigma^{\prime}}^{n}-\varphi_{\sigma}^{n}\right), \\
& \mathcal{R}_{32}^{(m)}=-\frac{1}{8} \sum_{n=0}^{N-1} \delta t \sum_{K=\left[\overrightarrow{\sigma \sigma^{\prime}}\right] \in \mathcal{M}} u_{\sigma}^{n} \tilde{u}_{\sigma}^{n+1}\left(\rho_{\sigma}^{n}+\rho_{K}^{n}\right)\left(\tilde{u}_{\sigma^{\prime}}^{n+1}-\tilde{u}_{\sigma}^{n+1}\right)\left(\varphi_{\sigma^{\prime}}^{n}-\varphi_{\sigma}^{n}\right) .
\end{aligned}
$$

Thanks to the regularity of $\varphi$, the Cauchy-Schwarz inequality yields:

$$
\left|\mathcal{R}_{31}^{(m)}\right| \leq C_{\varphi} h^{1 / 2}\left[\sum_{n=0}^{N-1} \delta t \sum_{K=\left[\overrightarrow{\sigma \sigma^{\prime}}\right] \in \mathcal{M}}\left|u_{\sigma}^{n}\right|\left(\rho_{\sigma}^{n}-\rho_{K}^{n}\right)^{2}\right]^{1 / 2}\left[\sum_{n=0}^{N-1} \delta t \sum_{K=\left[\overrightarrow{\sigma \sigma^{\prime}}\right] \in \mathcal{M}}|K|\left|u_{\sigma}^{n}\right|\left(\tilde{u}_{\sigma}^{n+1}\left(\tilde{u}_{\sigma^{\prime}}^{n+1}+\tilde{u}_{\sigma}^{n+1}\right)\right)^{2}\right]^{1 / 2},
$$

and thus, invoking the estimate (3.47),

$$
\left|\mathcal{R}_{31}^{(m)}\right| \leq C\left(h^{(m)}\right)^{1 / 2}\left[\left\|u^{(m)}\right\|_{L^{2}(\Omega \times(0, T))}+\left\|\tilde{u}^{(m)}\right\|_{L^{8}(\Omega \times(0, T))}^{4}\right] .
$$

Similarly, we get:

$$
\left|\mathcal{R}_{32}^{(m)}\right| \leq C_{\varphi} \frac{h}{\nu^{1 / 2}}\left[\sum_{n=0}^{N-1} \delta t \sum_{K=\left[\overrightarrow{\sigma \sigma^{\prime}}\right] \in \mathcal{M}} \frac{\nu}{h_{K}}\left(\tilde{u}_{\sigma^{\prime}}^{n+1}-\tilde{u}_{\sigma}^{n+1}\right)^{2}\right]^{1 / 2}\left[\sum_{n=0}^{N-1} \delta t \sum_{K=\left[\overrightarrow{\sigma \sigma^{\prime}}\right] \in \mathcal{M}}|K|\left(u_{\sigma}^{n} \tilde{u}_{\sigma}^{n+1}\left(\rho_{\sigma}^{n}+\rho_{K}^{n}\right)\right)^{2}\right]^{1 / 2},
$$


and thus:

$$
\left|\mathcal{R}_{32}^{(m)}\right| \leq C \frac{h^{(m)}}{\left(\nu^{(m)}\right)^{1 / 2}}\left[\left\|u^{(m)}\right\|_{L^{6}(\Omega \times(0, T))}^{3}+\left\|\tilde{u}^{(m)}\right\|_{L^{6}(\Omega \times(0, T))}^{3}+\left[\left\|\rho^{(m)}\right\|_{L^{6}(\Omega \times(0, T))}^{3}\right] .\right.
$$

Therefore, under the assumptions of the theorem, we have

$$
\lim _{m \longrightarrow+\infty} T_{3}^{(m)}=-\frac{1}{2} \int_{0}^{T} \int_{\Omega} \bar{\rho} \bar{u}^{3} \partial_{x} \varphi \mathrm{d} x \mathrm{~d} t .
$$

Together with the results which were obtained in the proof of Theorem 3.10, this yields

$$
\lim _{m \longrightarrow+\infty}\left(\sum_{i=1}^{5} T_{i}^{(m)}+R^{(m)}\right) \geq-\int_{0}^{T} \int_{\Omega}\left[\eta \partial_{t} \varphi+(\eta+p) u \partial_{x} \varphi\right] \mathrm{d} x \mathrm{~d} t-\int_{\Omega} \eta_{0}(x) \varphi(x, 0) \mathrm{d} x .
$$

In the proof of Theorem 3.10, we obtained that $\lim _{m \rightarrow+\infty} R^{(m)} \geq 0$. Furthermore, Lemma 3.16 implies that $\lim _{m \rightarrow+\infty} P^{(m)}=0$, which concludes the proof of the theorem.

\section{The PRESSURE CORRECTION SCHEME FOR THE Full Euler EQUATIONS}

We now turn to the development and study of a similar pressure correction scheme for the full Euler equations, that is for the system (1.2) assuming $\boldsymbol{\tau}(\boldsymbol{u})=0$. Numerical schemes for the Euler equations have been widely studied, and are very often based on Riemann solvers on the system consisting of the mass balance, the momentum balance, and the total energy balance. We start this section by explaining why we use the internal energy balance rather the the total energy in the correction scheme, and the precautions that must be taken in order to compute correct shocks in this way.

\subsection{Internal energy, kinetic energy and total energy}

Let us suppose that the solution to the Navier-Stokes equations (1.2) is regular. As already mentioned, taking the inner product of the momentum balance equation (1.2b) by $\boldsymbol{u}$ and using the mass balance equation, we obtain the so-called kinetic energy balance equation:

$$
\frac{1}{2} \partial_{t}\left(\rho|\boldsymbol{u}|^{2}\right)+\frac{1}{2} \operatorname{div}\left(\rho|\boldsymbol{u}|^{2} \boldsymbol{u}\right)+\nabla p \cdot \boldsymbol{u}=\operatorname{div}(\boldsymbol{\tau}(\boldsymbol{u})) \cdot \boldsymbol{u} .
$$

Subtracting this relation from the total energy balance, we obtain the internal energy balance equation:

$$
\partial_{t}(\rho e)+\operatorname{div}(\rho e \boldsymbol{u})+p \operatorname{div}(\boldsymbol{u})=\boldsymbol{\tau}(\boldsymbol{u}): \nabla \boldsymbol{u} .
$$

Since,

- from thermodynamical arguments, $\boldsymbol{\tau}(\boldsymbol{u}): \boldsymbol{\nabla u} \geq 0$;

- thanks to the mass balance equation, the first two terms in the left-hand side of (4.2) may be recast as a transport operator: $\partial_{t}(\rho e)+\operatorname{div}(\rho e \boldsymbol{u})=\rho\left[\partial_{t} e+\boldsymbol{u} \cdot \nabla e\right]$;

- and, finally, from the equation of state, the pressure vanishes when $e=0$,

this equation implies that, if $e \geq 0$ at $t=0$ and with suitable boundary conditions, then $e$ remains non-negative at all times.

Our aim here is to build a scheme stable and accurate at all Mach numbers, and, in particular, which boils down to a usual scheme for incompressible flows (or, more generally speaking, for the asymptotic model of vanishing Mach number flows [45]) when the Mach number tends to zero. In these latter models, the natural energy balance equation is the internal energy equation (4.2). In addition, discretizing (4.2) instead of the total energy balance (1.2c) presents two advantages: 
- first, it avoids the space discretization of the total energy, which is rather unnatural for staggered schemes since the degrees of freedom for the velocity and the scalar variables are not colocated,

- second, a suitable discretization of (4.2) may yield, "by construction" of the scheme, the positivity of the internal energy [18].

However, in the inviscid case and for solutions with shocks, equation (4.2) (with $\tau=0$ ) is not equivalent to the conservative total energy balance (1.2c) (with $\boldsymbol{\tau}=0$ ); more precisely speaking, at the locations of shocks, positive measures should replace, at the right-hand side of equation (4.2), the term $\boldsymbol{\tau}(\boldsymbol{u}): \boldsymbol{\nabla} \boldsymbol{u}$ which is formally the product of vanishing quantities (for a Newtonian fluid, the viscosity) and infinite derivatives of the velocity. Discretizing (4.2) instead of (1.2c) may thus yield a scheme which does not compute the correct weak discontinuous solutions; in particular, the numerical solutions may present (smeared) shocks which do not satisfy the Rankine-Hugoniot conditions associated with (1.2c). The essential result of this section is to provide a solution to circumvent this problem. To this purpose, we closely mimic the above performed formal computation:

- Starting from the discrete momentum balance equation, we derive a discrete kinetic energy balance (in fact, this computation is already performed in the previous sections, since we use for the full Euler equations the same discrete momentum balance as in the barotropic case). In this relation, residual terms which do no tend to zero with space and time step appear (they are the discrete manifestations of the above mentioned measures).

- These residual terms are then compensated by corrective terms in the internal energy balance.

We provide a theoretical justification of this process by showing that, in the $1 \mathrm{D}$ case, if the scheme is stable and converges to a limit (in a sense to be defined), this limit satisfies a weak form of (1.2c) which implies the correct Rankine-Hugoniot conditions. Then, we perform numerical tests which substantiate this analysis. A fully implicit scheme was studied in [26] along with two pressure correction schemes: the first correction scheme is appealing for its (relative) simplicity, but does not seem to warrant the sign of the internal energy (so that the unconditional stability induced by the above mentioned conservation of the total energy property is lost); the second scheme cures this problem, at the price of the introduction of an additional elliptic problem which must be solved at the beginning of each time step to determine a tentative pressure. Here, we present a variant of these schemes, that preserves the positivity of the internal energy thanks to a renormalization step which only consists in a weighting of the discrete pressure gradient and does not require any elliptic solve (and which is thus much less costly). As the correction scheme for barotropic flows, it is implemented in the industrial open-source code $\mathrm{CALIF}^{3} \mathrm{~S}[5]$. Let us mention also that fully explicit versions have been studied [30,31].

\subsection{The scheme}

We propose in this section a pressure correction scheme, which, as in the barotropic case, features a renormalization step for the pressure gradient. As previously mentioned, we add a corrective term in the internal energy equation; we are able to show that this corrective term is non negative, which ensures the positivity of the internal energy and the existence of a solution to the scheme.

With the notations that were introduced in Section 3.2.1, the algorithm reads, for $0 \leq n \leq N-1$ :

\section{Pressure gradient renormalization step:}

$$
\forall \sigma \in \mathcal{E}, \quad(\widetilde{\nabla} p)_{\sigma}^{n+1}=\sqrt{\frac{\rho_{D_{\sigma}}^{n}}{\rho_{D_{\sigma}}^{n-1}}}(\nabla p)_{\sigma}^{n} .
$$


Prediction step - Solve for $\tilde{\boldsymbol{u}}^{n+1}$ :

For $1 \leq i \leq d, \quad \forall \sigma \in \mathcal{E}_{\mathcal{S}}^{(i)}$

$$
\frac{\left|D_{\sigma}\right|}{\delta t}\left(\rho_{D_{\sigma}}^{n} \tilde{u}_{\sigma, i}^{n+1}-\rho_{D_{\sigma}}^{n-1} u_{\sigma, i}^{n}\right)+\sum_{\epsilon \in \overline{\mathcal{E}}\left(D_{\sigma}\right)} F_{\sigma, \epsilon}^{n} \tilde{u}_{\epsilon, i}^{n+1}-\left|D_{\sigma}\right|\left(\Delta^{\mathcal{M}} \tilde{\boldsymbol{u}}\right)_{\sigma, i}^{n+1}+\left|D_{\sigma}\right|(\widetilde{\nabla p})_{\sigma, i}^{n+1}=0
$$

Correction step - Solve for $\rho^{n+1}, p^{n+1}, e^{n+1}$ and $\boldsymbol{u}^{n+1}$ :

For $1 \leq i \leq d, \quad \forall \sigma \in \mathcal{E}_{\mathcal{S}}^{(i)}$,

$$
\begin{aligned}
& \frac{\left|D_{\sigma}\right|}{\delta t} \rho_{D_{\sigma}}^{n}\left(u_{\sigma, i}^{n+1}-\tilde{u}_{\sigma, i}^{n+1}\right)+\left|D_{\sigma}\right|\left[(\boldsymbol{\nabla} p)_{\sigma, i}^{n+1}-(\widetilde{\nabla p})_{\sigma, i}^{n+1}\right]=0, \\
& \forall K \in \mathcal{M}, \quad \frac{|K|}{\delta t}\left(\rho_{K}^{n+1}-\rho_{K}^{n}\right)+\sum_{\sigma \in \mathcal{E}(K)} F_{K, \sigma}^{n+1}=0, \\
& \forall K \in \mathcal{M}, \quad \frac{|K|}{\delta t}\left(\rho_{K}^{n+1} e_{K}^{n+1}-\rho_{K}^{n} e_{K}^{n}\right)+\sum_{\sigma \in \mathcal{E}(K)} \begin{array}{c}
F_{K, \sigma}^{n+1} e_{\sigma}^{n+1} \\
+|K| p_{K}^{n+1}(\operatorname{div} \boldsymbol{u})_{K}^{n+1}=S_{K}^{n+1},
\end{array}
\end{aligned}
$$

$\forall K \in \mathcal{M}, \quad p_{K}^{n+1}=(\gamma-1) \rho_{K}^{n+1} e_{K}^{n+1}$,

where we make an upwind choice for $e$ (again a crucial choice to ensure the positivity of the internal energy):

$$
\text { for } \sigma=K\left|L \in \mathcal{E}_{\mathrm{int}}, \quad e_{\sigma}^{n+1}=\right| \begin{array}{ll}
e_{K}^{n+1} & \text { if } F_{K, \sigma}^{n+1} \geq 0 \\
e_{L}^{n+1} & \text { otherwise }
\end{array}
$$

The initialization of the scheme is performed in a way similar to the barotropic case. First, $\rho^{-1}, e^{0}$ and $\boldsymbol{u}^{0}$ are given by the average of the initial conditions $\rho_{0}, e_{0}$ and $\boldsymbol{u}_{0}$, i.e. by (3.43) and the following relation:

$$
\forall K \in \mathcal{M}, \quad e_{K}^{0}=\frac{1}{|K|} \int_{K} e_{0}(\boldsymbol{x}) \mathrm{d} \boldsymbol{x} .
$$

Then, we compute $\rho^{0}$ by solving the mass balance equation (4.3d). Finally, the initial pressure $p^{0}$ is computed from the initial density $\rho^{0}$ by the equation of state: $\forall K \in \mathcal{M}, p_{K}^{0}=(\gamma-1) \rho_{K}^{0} e_{K}^{0}$. As in the barotropic case, the objective of this procedure is to perform the first prediction step with $\left(\rho_{D_{\sigma}}^{-1}\right)_{\sigma \in \mathcal{E}},\left(\rho_{D_{\sigma}}^{0}\right)_{\sigma \in \mathcal{E}}$ and the dual mass fluxes satisfying the mass balance.

There only remains to define the corrective terms $S_{K}^{n+1}$ in the internal energy balance (4.3e), with the aim to recover a consistent discretization of the total energy balance. The first idea to do this could be just to sum the (discrete) kinetic energy balance with the internal energy balance: it is indeed possible for a colocated discretization. But here, we face the fact that the kinetic energy balance is associated with the dual mesh, while the internal energy balance is discretized on the primal one. The way to circumvent this difficulty is to remark that we do not really need a discrete total energy balance; in fact, we only need to recover (a weak form of) this equation when the mesh and time steps tend to zero. To this purpose, we choose the quantities $\left(S_{K}^{n+1}\right)$ in such a way as to somewhat compensate the terms $\left(R_{\sigma, i}^{n+1}\right)$ defined by (3.45) appearing at the right-hand-side of the discrete kinetic energy identity (3.44):

$$
\forall K \in \mathcal{M}, S_{K}^{n+1}=\sum_{i=1}^{d} S_{K, i}^{n+1},
$$


with:

$$
S_{K, i}^{n+1}=\frac{1}{2} \rho_{K}^{n-1} \sum_{\sigma \in \mathcal{E}(K) \cap \mathcal{E}_{\mathcal{S}}^{(i)}} \frac{\left|D_{K, \sigma}\right|}{\delta t}\left(\tilde{u}_{\sigma, i}^{n+1}-u_{\sigma, i}^{n}\right)^{2}+\sum_{\substack{\epsilon \in \overline{\mathcal{E}}_{\mathcal{S}}^{(i)}, \epsilon \cap \bar{K} \neq \emptyset, \epsilon=D_{\sigma} \mid D_{\sigma^{\prime}}}} \alpha_{K, \epsilon} \nu h_{\epsilon}^{d-2}\left(\tilde{u}_{\sigma, i}^{n+1}-\tilde{u}_{\sigma^{\prime}, i}^{n+1}\right)^{2} .
$$

The coefficient $\alpha_{K, \epsilon}$ is fixed to 1 if the face $\epsilon$ is included in $K$, and this is the only situation to consider for the RT and CR discretizations. For the MAC scheme, some dual faces are included in the primal cells, but some lie on their boundary; for such a dual face $\epsilon$, we denote by $\mathcal{N}_{\epsilon}$ the set of cells $M$ such that $\bar{M} \cap \epsilon \neq \emptyset$ (the cardinal of this set being always 4 ), and compute $\alpha_{K, \epsilon}$ by:

$$
\alpha_{K, \epsilon}=\frac{|K|}{\sum_{M \in \mathcal{N}_{\epsilon}}|M|} .
$$

For a uniform grid, this formula yields $\alpha_{K, \epsilon}=1 / 4$.

The expression of the terms $\left(S_{K}^{n+1}\right)_{K \in \mathcal{M}}$ is justified by the passage to the limit in the scheme (for a onedimensional problem) performed in Section 4.3. However, its expression may be anticipated, thanks to the following remarks. First, we note that:

$$
\sum_{K \in \mathcal{M}} S_{K}^{n+1}-\sum_{i=1}^{d} \sum_{\sigma \in \mathcal{E}_{\mathcal{S}}^{(i)}} R_{\sigma, i}^{n+1}=0
$$

Indeed, for $K \in \mathcal{M}$ and $\sigma=K \mid L$, the first part of $S_{K, i}^{n+1}$, thanks to the expression (3.4) of the density at the face $\rho_{D_{\sigma}}^{n}$, results from a dispatching of the first part of the kinetic energy balance residual $R_{\sigma, i}^{n+1}$ over the two cells adjacent to $\sigma$ :

$$
\frac{1}{2} \frac{\left|D_{\sigma}\right|}{\delta t} \rho_{D_{\sigma}}^{n-1}\left(\tilde{u}_{\sigma, i}^{n+1}-u_{\sigma, i}^{n}\right)^{2}=\underbrace{\frac{1}{2} \frac{\left|D_{K, \sigma}\right|}{\delta t} \rho_{K}^{n-1}\left(\tilde{u}_{\sigma, i}^{n+1}-u_{\sigma, i}^{n}\right)^{2}}_{\text {affected to } K}+\underbrace{\frac{1}{2} \frac{\left|D_{L, \sigma}\right|}{\delta t} \rho_{L}^{n-1}\left(\tilde{u}_{\sigma, i}^{n+1}-u_{\sigma, i}^{n}\right)^{2}}_{\text {affected to } L} .
$$

For the second part of the remainder, a standard reordering of the sum yields:

$$
\sum_{i=1}^{d} \sum_{\sigma \in \mathcal{E}_{\mathcal{S}}^{(i)}}\left[\sum_{\epsilon=D_{\sigma} \mid D_{\sigma^{\prime}}} \nu h_{\epsilon}^{d-2}\left(\tilde{u}_{\sigma, i}^{n+1}-\tilde{u}_{\sigma^{\prime}, i}^{n+1}\right)\right] \tilde{u}_{\sigma, i}^{n+1}=\sum_{i=1}^{d} \sum_{\epsilon=D_{\sigma} \mid D_{\sigma^{\prime}} \in \overline{\mathcal{E}}_{\mathcal{S}}^{(i)}} \nu h_{\epsilon}^{d-2}\left(\tilde{u}_{\sigma, i}^{n+1}-\tilde{u}_{\sigma^{\prime}, i}^{n+1}\right)^{2} .
$$

One may wonder why we do not use in $S_{K}^{n+1}$ the expression of this term as it is written in the remainder $R_{\sigma, i}^{n+1}$, i.e., in other words, use the numerical diffusion multiplied by $\boldsymbol{u}$ instead of the dissipation. A first answer is that we mimic what happens at the continuous level: the term which appears in the kinetic energy balance is $\operatorname{div}(\boldsymbol{\tau}(\boldsymbol{u})) \cdot \boldsymbol{u}$ and the corresponding term in the internal energy balance is the dissipation $\boldsymbol{\tau}(\boldsymbol{u}): \boldsymbol{\nabla} \boldsymbol{u}$. A more involved argument is that the expression in $S_{K}^{n+1}$ provides a positive source term to the internal energy balance, and we may hope that the difference between the numerical diffusion multiplied by $\boldsymbol{u}$ and the associated dissipation tends to zero (because the numerical diffusion tends to zero) in the sense of distributions. To have an intuition of this fact, let us consider the toy elliptic problem, posed over $\Omega$ :

$$
v-\nu \Delta v=f,
$$

where $\nu$ is a positive parameter and $f \in \mathrm{L}^{2}(\Omega)$. Assuming homogeneous Dirichlet boundary conditions, we obtain by standard variational arguments $\|v\|_{L^{2}(\Omega)}+\nu^{1 / 2}\|\nabla v\|_{L^{2}(\Omega)^{d}} \leq C$, with $C$ only depending on $\Omega$ and $f$. We thus get, with $\varphi \in \mathrm{C}_{c}^{\infty}(\Omega)$ :

$$
\int_{\Omega}\left[\nu(\Delta v) v+\nu|\nabla v|^{2}\right] \varphi \mathrm{d} \boldsymbol{x}=\nu \int_{\Omega} \operatorname{div}(v \boldsymbol{\nabla} v) \varphi \mathrm{d} \boldsymbol{x}=-\nu \int_{\Omega} v \boldsymbol{\nabla} v \cdot \boldsymbol{\nabla} \varphi \mathrm{d} \boldsymbol{x},
$$


and so, finally, by the Cauchy-Schwarz inequality:

$$
\left|\int_{\Omega}\left[\nu(\Delta v) v+\nu|\nabla v|^{2}\right] \varphi \mathrm{d} \boldsymbol{x}\right| \leq C\|\nabla \varphi\|_{L^{\infty}(\Omega)^{d}} \nu^{1 / 2}
$$

so this term tends to zero if so does $\nu$. A discrete analogue of this simple computation is used to pass to the limit in the scheme in the next section (with a control on the unknown assumed and not proven).

Note that the term $S_{K}^{n+1}$ is non-negative. Consequently, adapting the proof of ([18], Thm. 4.1) to cope with this additional term, we obtain that the scheme admits at least one solution, which satisfies $p \geq 0, \rho \geq 0$ and $e \geq 0$. In addition, equation (4.7) shows that the scheme conserves the integral of the total energy over the computational domain.

Theorem 4.1 (Existence and stability). Assume that for all $K \in \mathcal{M}, e_{K}^{0}>0$ and $\rho_{K}^{-1}>0$. Then there exists a solution to the scheme (4.3), which furthermore satisfies $\rho_{K}^{0}>0$ and, for $1 \leq n \leq N$ and $K \in \mathcal{M}, e_{K}^{n}>0$, $\rho_{K}^{n}>0$, and the following discrete analogue of the total energy balance:

$$
\begin{aligned}
\sum_{K \in \mathcal{M}}|K| e_{K}^{n} & +\frac{1}{2} \sum_{i=1}^{d} \sum_{\sigma \in \mathcal{E}_{\mathcal{S}}^{(i)}}\left|D_{\sigma}\right| \rho_{D_{\sigma}}^{n-1}\left(u_{\sigma, i}^{n}\right)^{2}+\mathcal{R}^{n} \\
& \leq \sum_{K \in \mathcal{M}}|K| e_{K}^{0}+\frac{1}{2} \sum_{i=1}^{d} \sum_{\sigma \in \mathcal{E}_{\mathcal{S}}^{(i)}}\left|D_{\sigma}\right| \rho_{D_{\sigma}}^{-1}\left(u_{\sigma, i}^{0}\right)^{2}+\mathcal{R}^{0}
\end{aligned}
$$

where:

$$
\mathcal{R}^{n}=\delta t^{2} \sum_{\sigma \in \mathcal{E}_{\text {int }}} \frac{\left|D_{\sigma}\right|}{\rho_{D_{\sigma}}^{n-1}}\left|(\nabla p)_{\sigma}^{n}\right|^{2}=\delta t^{2} \sum_{\sigma=K \mid L \in \mathcal{E}_{\text {int }}} \frac{|\sigma|^{2}}{\left|D_{\sigma}\right| \rho_{D_{\sigma}}^{n-1}}\left(p_{K}^{n}-p_{L}^{n}\right)^{2}
$$

\subsection{Passing to the limit in the scheme (1D case)}

We now undertake the passage to the limit in the scheme (4.3) in the one-dimensional case. Using the notations (3.25)-(3.27), the scheme may be rewritten in "one-dimensional notations" as follows:

Initialization - Compute $\rho^{-1}, u^{0}$ and $\rho^{0}$ by (3.53a) and $e^{0}$ and $p^{0}$ by:

$$
\begin{aligned}
& \forall K \in \mathcal{M}, e_{K}^{0}=\frac{1}{|K|} \int_{K} e_{0}(x) \mathrm{d} x, \\
& \forall K \in \mathcal{M}, p_{K}^{0}=(\gamma-1) \rho_{K}^{0} e_{K}^{0} .
\end{aligned}
$$

\section{Pressure gradient renormalization step}

$$
\forall \sigma=K \mid L \in \mathcal{E}_{\mathrm{int}}, \quad(\widetilde{\delta p})_{\sigma}^{n+1}=\sqrt{\frac{\rho_{D_{\sigma}}^{n}}{\rho_{D_{\sigma}}^{n-1}}}\left(p_{K}^{n}-p_{L}^{n}\right) .
$$

Prediction step - Solve for $\tilde{u}^{n+1}$ :

$$
\forall \sigma=\overrightarrow{K \mid L} \in \mathcal{E}_{\text {int }}, \quad \frac{\left|D_{\sigma}\right|}{\delta t}\left(\rho_{D_{\sigma}}^{n} \tilde{u}_{\sigma}^{n+1}-\rho_{D_{\sigma}}^{n-1} u_{\sigma}^{n}\right)+F_{L}^{n} \tilde{u}_{L}^{n+1}-F_{K}^{n} \tilde{u}_{K}^{n+1}-\left|D_{\sigma}\right|\left(\Delta_{\mathcal{M}} \tilde{u}\right)_{\sigma}^{n+1}+(\tilde{\delta p})_{\sigma}^{n+1}=0 .
$$


Correction step - Solve for $\rho^{n+1}, p^{n+1}, e^{n+1}$ and $u^{n+1}$ :

$$
\begin{array}{ll}
\forall \sigma=\overrightarrow{K \mid L} \in \mathcal{E}_{\text {int }}, \quad \frac{\left|D_{\sigma}\right|}{\delta t} \rho_{D_{\sigma}}^{n}\left(u_{\sigma}^{n+1}-\tilde{u}_{\sigma}^{n+1}\right)+\left(p_{L}^{n+1}-p_{K}^{n+1}\right)-(\widetilde{\delta p})_{\sigma}^{n+1}=0, & \begin{array}{ll}
\forall K=\left[\overrightarrow{\sigma \sigma^{\prime}}\right] \in \mathcal{M}, & \frac{|K|}{\delta t}\left(\rho_{K}^{n+1}-\rho_{K}^{n}\right)+F_{\sigma^{\prime}}^{n+1}-F_{\sigma}^{n+1}=0, \\
\forall K=\left[\overrightarrow{\sigma \sigma^{\prime}}\right] \in \mathcal{M}, & \frac{|K|}{\delta t}\left(\rho_{K}^{n+1} e_{K}^{n+1}-\rho_{K}^{n} e_{K}^{n}\right)+F_{\sigma^{\prime}}^{n+1} e_{\sigma^{\prime}}^{n+1}-F_{\sigma}^{n+1} e_{\sigma}^{n+1} \\
\forall K \in \mathcal{M}, & +p_{K}^{n+1}\left(u_{\sigma^{\prime}}^{n+1}-u_{\sigma}^{n+1}\right)=S_{K}^{n+1},
\end{array} \\
\forall p_{K}^{n+1}=(\gamma-1) \rho_{K}^{n+1} e_{K}^{n+1} .
\end{array}
$$

The corrective term $S_{K}^{n+1}$ reads:

$$
\forall K=\left[\sigma \sigma^{\prime}\right], \quad S_{K}^{n+1}=\frac{|K|}{4 \delta t} \rho_{K}^{n-1}\left[\left(\tilde{u}_{\sigma}^{n+1}-u_{\sigma}^{n}\right)^{2}+\left(\tilde{u}_{\sigma^{\prime}}^{n+1}-u_{\sigma^{\prime}}^{n}\right)^{2}\right]+\frac{\nu}{h_{K}}\left(\tilde{u}_{\sigma}^{n+1}-\tilde{u}_{\sigma^{\prime}}^{n+1}\right)^{2} .
$$

For discrete functions $q$ and $v$ defined on the primal and dual mesh, respectively, we define a discrete $\mathrm{L}^{1}((0, T) ; \mathrm{B} V(\Omega))$ norm by:

$$
\|q\|_{\mathcal{T}, x, \mathrm{~B} V}=\sum_{n=0}^{N} \delta t \sum_{\sigma=K \mid L \in \mathcal{E}_{\mathrm{int}}}\left|q_{L}^{n}-q_{K}^{n}\right|, \quad\|v\|_{\mathcal{T}, x, \mathrm{~B} V}=\sum_{n=0}^{N} \delta t \sum_{\epsilon=D_{\sigma} \mid D_{\sigma^{\prime}} \in \overline{\mathcal{E}}_{\mathrm{int}}}\left|v_{\sigma^{\prime}}^{n}-v_{\sigma}^{n}\right|,
$$

and a discrete $\mathrm{L}^{1}(\Omega ; \mathrm{B} V((0, T)))$ norm by:

$$
\|q\|_{\mathcal{T}, t, \mathrm{~B} V}=\sum_{K \in \mathcal{M}}|K| \sum_{n=0}^{N-1}\left|q_{K}^{n+1}-q_{K}^{n}\right|, \quad\|v\|_{\mathcal{T}, t, \mathrm{~B} V}=\sum_{\sigma \in \mathcal{E}}\left|D_{\sigma}\right| \sum_{n=0}^{N-1}\left|v_{\sigma}^{n+1}-v_{\sigma}^{n}\right| .
$$

For the proof of the consistency of the scheme (i.e. the proof of the Theorem 4.3 below), we need to introduce the following stability assumptions on a sequence $\left(\rho^{(m)}, p^{(m)}, e^{(m)}, \tilde{u}^{(m)}, u^{(m)}\right)_{m \in \mathbb{N}}$ of discrete solutions:

$$
\begin{aligned}
& \left|\left(\rho^{(m)}\right)_{K}^{n}\right|+\left|\left(p^{(m)}\right)_{K}^{n}\right|+\left|\left(e^{(m)}\right)_{K}^{n}\right| \leq C, \quad \forall K \in \mathcal{M}^{(m)}, \text { for } 0 \leq n \leq N^{(m)}, \forall m \in \mathbb{N}, \\
& \frac{1}{\left|\left(\rho^{(m)}\right)_{K}^{n}\right|} \leq C, \quad \forall K \in \mathcal{M}^{(m)}, \text { for } 0 \leq n \leq N^{(m)}, \forall m \in \mathbb{N}, \\
& \left|\left(u^{(m)}\right)_{\sigma}^{n}\right|+\left|\left(\tilde{u}^{(m)}\right)_{\sigma}^{n}\right| \leq C, \quad \forall \sigma \in \mathcal{E}^{(m)}, \text { for } 0 \leq n \leq N^{(m)}, \forall m \in \mathbb{N}, \\
& \left\|\rho^{(m)}\right\|_{\mathcal{T}, x, \mathrm{~B} V}+\left\|e^{(m)}\right\|_{\mathcal{T}, x, \mathrm{~B} V}+\left\|p^{(m)}\right\|_{\mathcal{T}, x, \mathrm{~B} V}+\left\|\tilde{u}^{(m)}\right\|_{\mathcal{T}, x, \mathrm{~B} V} \leq C, \quad \forall m \in \mathbb{N}, \\
& \left\|\rho^{(m)}\right\|_{\mathcal{T}, t, \mathrm{~B} V}+\left\|u^{(m)}\right\|_{\mathcal{T}, t, \mathrm{~B} V} \leq C, \quad \forall m \in \mathbb{N} .
\end{aligned}
$$

Note that we do not suppose any control on time discrete derivatives of $\tilde{u}^{(m)}$ (i.e. on $\left\|\tilde{u}^{(m)}\right\|_{\mathcal{T}, t, \mathrm{~B} V}$ ) and on the discrete space derivatives of $u^{(m)}$ (i.e. on $\left\|u^{(m)}\right\|_{\mathcal{T}, x, \mathrm{~B} V}$ ). Note also that, by definition of the initial conditions of the scheme, these inequalities imply that the functions $\rho_{0}, e_{0}$ and $u_{0}$ belong to $\mathrm{L}^{\infty}(\Omega) \cap \mathrm{B} V(\Omega)$. As in the barotropic case, we are not able to prove (4.10) for the solutions of the scheme; however, such inequalities are satisfied by the "interpolation" (for instance, by taking the cell average) of the solution to a Riemann problem, and are also observed in computations.

The following definition gathers the assumptions on the discretization which are needed in the proof of Theorem 4.3 below. 
Definition 4.2 (Regular sequence of discretizations). We define a regular sequence of discretizations $\left(\mathcal{M}^{(m)}, \delta t^{(m)}, \nu^{(m)}\right)_{m \in \mathbb{N}}$ as a sequence of meshes, time steps and numerical diffusion coefficients which are assumed to satisfy the following conditions:

(i) both the time step $\delta t^{(m)}$ and the size $h^{(m)}$ of the mesh $\mathcal{M}^{(m)}$ tend to zero as $m \rightarrow+\infty$;

(ii) there exists $C \in \mathbb{R}_{+}$(not necessarily lower than 1) such that the following CFL-like condition holds:

$$
\forall m \in \mathbb{N}, \quad \frac{\delta t^{(m)}}{\underline{\mathrm{h}}^{(m)}} \leq C, \quad \text { with } \underline{\mathrm{h}}^{(m)}=\min _{\sigma=K \mid L \in \mathcal{E}_{\text {int }}^{(m)}} \frac{1}{2}\left(h_{K}+h_{L}\right) ;
$$

(iii) there exists $C \in \mathbb{R}_{+}$such that the sequence of numerical diffusion coefficients $\left(\nu^{(m)}\right)_{m \in \mathbb{N}}$ satisfies $\lim _{m \rightarrow+\infty} \frac{\nu^{(m)}}{\underline{\mathrm{h}}^{(m)}} \leq C$.

A weak solution to the continuous problem satisfies, for any $\varphi \in \mathrm{C}_{c}^{\infty}([0, T) \times \Omega)$ :

$$
\begin{aligned}
& -\int_{\Omega \times(0, T)}\left[\rho \partial_{t} \varphi+\rho u \partial_{x} \varphi\right] \mathrm{d} x \mathrm{~d} t-\int_{\Omega} \rho_{0}(x) \varphi(x, 0) \mathrm{d} x=0, \\
& -\int_{\Omega \times(0, T)}\left[\rho u \partial_{t} \varphi+\left(\rho u^{2}+p\right) \partial_{x} \varphi\right] \mathrm{d} x \mathrm{~d} t-\int_{\Omega} \rho_{0}(x) u_{0}(x) \varphi(x, 0) \mathrm{d} x=0, \\
& -\int_{\Omega \times(0, T)}\left[\rho E \partial_{t} \varphi+(\rho E+p) u \partial_{x} \varphi\right] \mathrm{d} x \mathrm{~d} t-\int_{\Omega} \rho_{0}(x) E_{0}(x) \varphi(x, 0) \mathrm{d} x=0, \\
& p=(\gamma-1) \rho e, \quad E=\frac{1}{2} u^{2}+e, \quad E_{0}=\frac{1}{2} u_{0}^{2}+e_{0} .
\end{aligned}
$$

As in the barotropic case, these relations are not sufficient to define a weak solution to the problem, since they do not imply anything about the boundary conditions, but they allow to derive the Rankine-Hugoniot conditions.

We are now in position to state the following consistency result.

Theorem 4.3. Let $\Omega$ be an open bounded interval of $\mathbb{R}$. Let $\left(\mathcal{M}^{(m)}, \delta t^{(m)}, \nu^{(m)}\right)_{m \in \mathbb{N}}$ be a regular sequence of discretizations in the sense of Definition 4.2. Let $\left(\rho^{(m)}, p^{(m)}, e^{(m)}, \tilde{u}^{(m)}, u^{(m)}\right)_{m \in \mathbb{N}}$ be the corresponding sequence of solutions. We suppose that this sequence satisfies the bounds (4.10) and converges in $\mathrm{L}^{p}(\Omega \times(0, T))^{5}$, for $1 \leq p<+\infty$, to $(\bar{\rho}, \bar{p}, \bar{e}, \overline{\tilde{u}}, \bar{u}) \in \mathrm{L}^{\infty}(\Omega \times(0, T))^{5}$.

Then $\overline{\tilde{u}}=\bar{u}$ and $(\bar{\rho}, \bar{p}, \bar{e}, \bar{u})$ satisfies the system (4.12).

Proof. Let us first check that $\overline{\tilde{u}}=\bar{u}$. We first note that thanks to assumptions (4.10a) and (4.10b), the pressure prediction step (4.8b) yields that $\left|(\widetilde{\delta p})_{\sigma}^{n+1}\right| \leq C\left|p_{K}^{n}-p_{L}^{n}\right|$. Therefore, from the expression of the correction step for the velocity (4.8d), we have, again using assumption (4.10b):

$$
\left\|u^{(m)}-\tilde{u}^{(m)}\right\|_{\mathrm{L}^{1}(\Omega \times(0, T))} \leq C \delta t\left\|p^{(m)}\right\|_{\mathcal{T}, x, \mathrm{~B} V}
$$

which, passing to the limit when $m \rightarrow+\infty$, yields the result.

We now observe that the stability assumptions (4.10) and the regularity assumptions for the discretization of Definition 4.2 are stronger than the hypotheses made in the barotropic case (see Def. 3.5, Rem. 3.19 and the assumptions of Thm. 3.20). In particular, combining $\mathrm{L}^{\infty}$, space BV estimates and the assumption (iii) of Definition 4.2 on the numerical diffusion coefficient, we can prove the same control on the space translates of $\rho$ and $\tilde{u}$ as provided by the remainder terms (3.48) of the inequality (3.47). Consequently, the passage to the limit in the scheme for the mass and momentum balance equations is the same as in the barotropic case. 
We thus only need to prove that $(\bar{\rho}, \bar{p}, \bar{e}, \bar{u})$ satisfies (4.12c). Let us first multiply the one dimensional version of the discrete kinetic energy equation (3.44) by $\delta t \varphi_{\sigma}^{n}$ and sum over the faces and the time steps. Similarly, we multiply the discrete internal energy equation (4.8f) by $\delta t \varphi_{K}^{n}$, and sum over the primal cells and the time steps. Summing the two obtained relations, we get $T_{1}^{(m)}+\widetilde{T}_{2}^{(m)}+T_{3}^{(m)}+\widetilde{T}_{4}^{(m)}+T_{5}^{(m)}=R^{(m)}$, where the terms $T_{1}^{(m)}$, $T_{3}^{(m)}$, and $T_{5}^{(m)}$ are defined by (3.37a)-(3.37c) in the proof of Theorem 3.20, and

$$
\begin{aligned}
\widetilde{T}_{2}^{(m)} & =\sum_{n=0}^{N-1} \delta t \sum_{K \in \mathcal{M}} \frac{|K|}{\delta t}\left[\rho_{K}^{n+1} e_{K}^{n+1}-\rho_{K}^{n} e_{K}^{n}\right] \varphi_{K}^{n}, \\
\widetilde{T}_{4}^{(m)} & =\sum_{n=0}^{N-1} \delta t \sum_{K=\left[\overrightarrow{\sigma \sigma^{\prime}}\right] \in \mathcal{M}}\left[F_{\sigma^{\prime}}^{n+1} e_{\sigma^{\prime}}^{n+1}-F_{\sigma}^{n+1} e_{\sigma}^{n+1}\right] \varphi_{K}^{n}, \\
\widetilde{R}^{(m)} & =-\sum_{n=0}^{N-1} \delta t \sum_{\sigma \in \mathcal{E}}\left(\widetilde{R}_{\sigma}^{n+1}-P_{\sigma}^{n+1}\right) \varphi_{\sigma}^{n}+\sum_{n=0}^{N-1} \delta t \sum_{K \in \mathcal{M}} S_{K}^{n+1} \varphi_{K}^{n}
\end{aligned}
$$

where, in the latter relation, the remainder term reads, for $\sigma=\overrightarrow{K \mid L} \in \mathcal{E}_{\text {int }}$ with $K=\left[\overrightarrow{\sigma^{\prime} \sigma}\right]$ and $L=\left[\overrightarrow{\sigma \sigma^{\prime \prime}}\right]$ :

$$
\widetilde{R}_{\sigma}^{n+1}=\frac{1}{2} \frac{\left|D_{\sigma}\right|}{\delta t} \rho_{D_{\sigma}}^{n-1}\left[\tilde{u}_{\sigma}^{n+1}-u_{\sigma}^{n}\right]^{2}+\tilde{u}_{\sigma}^{n+1}\left[\frac{\nu}{h_{K}}\left(\tilde{u}_{\sigma}^{n+1}-\tilde{u}_{\sigma^{\prime}}^{n+1}\right)+\frac{\nu}{h_{L}}\left(\tilde{u}_{\sigma}^{n+1}-\tilde{u}_{\sigma^{\prime \prime}}^{n+1}\right)\right]
$$

and where $P_{\sigma}^{n+1}$ is defined by (3.45), and $S_{K}^{n+1}$ by (4.9).

The study of the terms $T_{1}^{(m)}, T_{3}^{(m)}$ and $T_{5}^{(m)}$ has already been performed in the proof of Theorem 3.20. The study of the term $\widetilde{T}_{2}^{(m)}$ (resp. $\widetilde{T}_{4}^{(m)}$ ) is similar to that of the term $T_{2}^{(m)}$ (resp. $T_{4}^{(m)}$ ) of the proof of Theorem 3.20, replacing the elastic potential $\mathcal{H}(\rho)$ by the internal energy $e$. Finally, we have to deal with the term $\widetilde{R}^{(m)}$. Here, the situation is different from Theorem 3.20 since we have to prove that this term tends to zero whereas for the entropy inequality in the barotropic case, we only had to prove that it is non-negative at the limit of vanishing time and space steps. We split $\widetilde{R}^{(m)}$ into three terms: $\widetilde{R}^{(m)}=R_{\delta t}^{(m)}+R_{\Delta}^{(m)}+P^{(m)}$ and give the expression of each of these three terms hereafter. The first term reads:

$$
R_{\delta t}^{(m)}=-\frac{1}{2} \sum_{n=0}^{N-1} \sum_{\sigma \in \mathcal{E}}\left|D_{\sigma}\right| \rho_{D_{\sigma}}^{n-1}\left(\tilde{u}_{\sigma}^{n+1}-u_{\sigma}^{n}\right)^{2} \varphi_{\sigma}^{n}+\frac{1}{2} \sum_{n=0}^{N-1} \sum_{K \in \mathcal{M}} \sum_{\sigma \in \mathcal{E}(K)}\left|D_{K, \sigma}\right| \rho_{K}^{n-1}\left(\tilde{u}_{\sigma}^{n+1}-u_{\sigma}^{n}\right)^{2} \varphi_{K}^{n} .
$$

Thanks to the definition of the density on the faces, we get:

$$
R_{\delta t}^{(m)}=\frac{1}{2} \sum_{n=0}^{N-1} \sum_{K \in \mathcal{M}} \sum_{\sigma \in \mathcal{E}(K)}\left|D_{K, \sigma}\right| \rho_{K}^{n-1}\left(\tilde{u}_{\sigma}^{n+1}-u_{\sigma}^{n}\right)^{2}\left(\varphi_{K}^{n}-\varphi_{\sigma}^{n}\right),
$$

and therefore, thanks to the regularity of $\varphi$ :

$$
\left|R_{\delta t}^{(m)}\right| \leq C_{\varphi} h \sum_{n=0}^{N-1} \sum_{K \in \mathcal{M}} \sum_{\sigma \in \mathcal{E}(K)}\left|D_{K, \sigma}\right| \rho_{K}^{n-1}\left(\tilde{u}_{\sigma}^{n+1}-u_{\sigma}^{n}\right)^{2} .
$$

Using the assumed uniform bound in $\mathrm{L}^{\infty}(\Omega \times(0, T))$ for the sequence $\left(\rho^{(m)}\right)_{m \in \mathbb{N}}$, we obtain that the remainder $R_{\delta t}^{(m)}$ satisfies $\left|R_{\delta t}^{(m)}\right| \leq C h^{(m)}\left(R_{\delta t, 1}^{(m)}+R_{\delta t, 2}^{(m)}\right)$, where $C$ is a real positive number, independent of $m$ and

$$
R_{\delta t, 1}^{(m)}=\sum_{n=0}^{N-1} \sum_{\sigma \in \mathcal{E}_{\mathrm{int}}}\left|D_{\sigma}\right|\left(u_{\sigma}^{n+1}-u_{\sigma}^{n}\right)^{2}, \quad R_{\delta t, 2}^{(m)}=\sum_{n=0}^{N-1} \sum_{\sigma \in \mathcal{E}_{\mathrm{int}}}\left|D_{\sigma}\right|\left(u_{\sigma}^{n+1}-\tilde{u}_{\sigma}^{n+1}\right)^{2} .
$$


The first of this term satisfies

$$
\left|R_{\delta t, 1}^{(m)}\right| \leq 2\left\|u^{(m)}\right\|_{L^{\infty}(\Omega \times(0, T))}\left\|u^{(m)}\right\|_{\mathcal{T}, t, \mathrm{~B} V},
$$

and, thanks once again to the expression of the velocity correction step (4.8d), we have for the second one:

$$
\left|R_{\delta t, 2}^{(m)}\right| \leq C\left\|\frac{1}{\rho^{(m)}}\right\|_{\mathrm{L}^{\infty}(\Omega \times(0, T))}\left(\left\|u^{(m)}\right\|_{\mathrm{L}^{\infty}(\Omega \times(0, T))}+\left\|\tilde{u}^{(m)}\right\|_{\mathrm{L}^{\infty}(\Omega \times(0, T))}\right)\left\|p^{(m)}\right\|_{\mathcal{T}, x, \mathrm{~B} V}
$$

where $C$ is again a real positive number, independent of $m$. Hence $R_{\delta t}^{(m)}$ tends to zero as $m$ tends to $+\infty$. Let us now turn to $R_{\Delta}^{(m)}$, which reads:

$$
\begin{aligned}
R_{\Delta}^{(m)}= & -\sum_{n=0}^{N-1} \delta t \sum_{\substack{\sigma=\overrightarrow{K \mid L} \in \mathcal{E}_{\text {int }}, K=\left[\overrightarrow{\sigma^{\prime} \sigma}\right], L=\left[\overrightarrow{\sigma \sigma^{\prime \prime}}\right]}} \tilde{u}_{\sigma}^{n+1}\left[\frac{\nu}{h_{K}}\left(\tilde{u}_{\sigma}^{n+1}-\tilde{u}_{\sigma^{\prime}}^{n+1}\right)+\frac{\nu}{h_{L}}\left(\tilde{u}_{\sigma}^{n+1}-\tilde{u}_{\sigma^{\prime \prime}}^{n+1}\right)\right] \varphi_{\sigma}^{n} \\
& +\sum_{n=0}^{N-1} \delta t \sum_{K=\left[\sigma \sigma^{\prime}\right] \in \mathcal{M}} \frac{\nu}{h_{K}}\left(\tilde{u}_{\sigma}^{n+1}-\tilde{u}_{\sigma^{\prime}}^{n+1}\right)^{2} \varphi_{K}^{n} .
\end{aligned}
$$

As explained in Section 4.2, the general idea is now to recast this term as a discrete version of the integral over space and time of a quantity of the form $-u \partial_{x} u \partial_{x} \varphi$ scaled by a numerical viscosity vanishing with the space step; then, the supposed controls on the solution imply that the term tends to zero. Reordering the sums, we get:

$$
R_{\Delta}^{(m)}=\sum_{n=0}^{N-1} \delta t \sum_{K=\left[\overrightarrow{\left.\sigma \sigma^{\prime}\right]}\right.} \frac{\nu}{h_{K}}\left(\tilde{u}_{\sigma}^{n+1}-\tilde{u}_{\sigma^{\prime}}^{n+1}\right)\left(\tilde{u}_{\sigma^{\prime}}^{n+1} \varphi_{\sigma^{\prime}}^{n}-\tilde{u}_{\sigma}^{n+1} \varphi_{\sigma}^{n}\right)+\frac{\nu}{h_{K}}\left(\tilde{u}_{\sigma^{\prime}}^{n+1}-\tilde{u}_{\sigma}^{n+1}\right)^{2} \varphi_{K}^{n},
$$

and thus:

$$
R_{\Delta}^{(m)}=\sum_{n=0}^{N-1} \delta t \sum_{K=\left[\overrightarrow{\sigma \sigma^{\prime}}\right] \in \mathcal{M}} \frac{\nu}{h_{K}}\left(\tilde{u}_{\sigma}^{n+1}-\tilde{u}_{\sigma^{\prime}}^{n+1}\right)\left[\tilde{u}_{\sigma}^{n+1}\left(\varphi_{K}^{n}-\varphi_{\sigma}^{n}\right)+\tilde{u}_{\sigma^{\prime}}^{n+1}\left(\varphi_{\sigma^{\prime}}^{n}-\varphi_{K}^{n}\right)\right] .
$$

We thus get, thanks to the regularity of $\varphi$ :

$$
\left|R_{\Delta}^{(m)}\right| \leq \nu C_{\varphi}\left\|\tilde{u}^{(m)}\right\|_{L^{\infty}(\Omega \times(0, T))}\left\|\tilde{u}^{(m)}\right\|_{\mathcal{T}, x, \mathrm{~B} V},
$$

which yields the desired estimate. Finally, $P^{(m)}$ reads:

$$
P^{(m)}=\sum_{n=0}^{N-1} \delta t \sum_{\sigma \in \mathcal{E}} P_{\sigma}^{n+1} \varphi_{\sigma}^{n}
$$

and the fact that this term tends to zero is stated in Lemma 3.16. This concludes the proof.

\subsection{Numerical tests}

In this section, we assess the behaviour of the scheme on a one dimensional Riemann problem. We choose initial conditions such that the structure of the solution consists in two shock waves, separated by the contact discontinuity, with sufficiently strong shocks to allow an easy discrimination of correct numerical solutions. These initial conditions are those proposed in ([63], Chap. 4), for the test referred to as Test 5:

$$
\text { left state: }\left[\begin{array}{l}
\rho_{\text {left }} \\
u_{\text {left }} \\
p_{\text {left }}
\end{array}\right]=\left[\begin{array}{l}
5.99924 \\
19.5975 \\
460.894
\end{array}\right] \quad \text { right state: }\left[\begin{array}{l}
\rho_{\text {right }} \\
u_{\text {right }} \\
p_{\text {right }}
\end{array}\right]=\left[\begin{array}{c}
5.99242 \\
-6.19633 \\
46.0950
\end{array}\right] .
$$

The problem is posed over $\Omega=(-0.5,0.5)$, and the discontinuity is initially located at $x=0$. 
At the boundaries, since, in this test, the flow is entering the domain, the solution is prescribed (which, in fact, is unimportant, the solution being constant at any time in a sufficiently large neighborhood of these boundaries). Previous numerical experiments addressing barotropic flows [27] showed that, at least for one dimensional computations with schemes similar to the one under study here, it was not necessary to upwind the convection term in the momentum balance equation; consequently, we only employ a centered approximation of the velocity at the dual faces.

The computations are performed with the open-source software $\mathrm{CALIF}^{3} \mathrm{~S}[5]$.

The density fields obtained with $h=1 / 2000$ (or a number of cells $n=2000$ ) at $t=0.035$, with and without assembling the corrective source term in the internal energy balance $\left(S_{K}\right)_{K \in \mathcal{M}}$, together with the analytical solution, are shown on Figure 2. The density and the pressure obtained, still with and without corrective terms, for various meshes, are plotted on Figures 3 and 4 respectively. For these computations, we take $\delta t=h / 20$, which yields a cfl number, with respect to the material velocity only, close to one. The first conclusion is that both schemes seem to converge, but the corrective term is necessary to obtain the right solution. In this case, for instance, we obtain the correct intermediate state for the pressure and velocity up to four digits in the essential part of the corresponding zone:

$$
\begin{array}{ll}
\text { (analytical) intermediate state: } & {\left[\begin{array}{l}
p^{*} \\
u^{*}
\end{array}\right]=\left[\begin{array}{l}
1691.65 \\
8.68977
\end{array}\right] \text { for } x \in(0.028,0.428)} \\
\text { numerical results: } & \mid \begin{array}{l}
p \in(1691.6,1691.8) \\
u \in(8.689,8.690)
\end{array} \text { for } x \in(0.032,0.417) .
\end{array}
$$

Without a corrective term, one can check that the obtained solution is not a weak solution to the Euler system: indeed, the Rankine-Hugoniot condition applied to the total energy balance, with the states obtained numerically, yields a right shock speed slightly greater than the analytical solution one, while the same shock speed obtained numerically is clearly lower.

We also observe that the scheme is rather diffusive especially for contact discontinuities for which the beneficial compressive effect of the shocks does not apply. More accurate variants may certainly be derived, using for instance MUSCL-like techniques; this work is underway [17].

In order to check the consistency of the scheme, we give in the table below the $\mathrm{L}^{1}(\Omega)$-norm of the difference between the numerical and the exact solution (denoted $\left.\left(\rho_{\mathrm{ex}}, p_{\mathrm{ex}}, u_{\mathrm{ex}}\right)\right)$ at $t=0.035$, for various grids and still for $\delta t=h / 20$.

\begin{tabular}{lccc}
\hline$h$ & $\left\|\rho-\rho_{\mathrm{ex}}\right\|_{\mathrm{L}^{1}(\Omega)}$ & $\left\|p-p_{\mathrm{ex}}\right\|_{\mathrm{L}^{1}(\Omega)}$ & $\left\|u-u_{\mathrm{ex}}\right\|_{\mathrm{L}^{1}(\Omega)}$ \\
\hline $1 / 250$ & 0.0662 & 1.235 & 0.00911 \\
$1 / 500$ & 0.0452 & 0.619 & 0.00437 \\
$1 / 1000$ & 0.0313 & 0.365 & 0.00232 \\
$1 / 2000$ & 0.0215 & 0.170 & 0.00125 \\
$1 / 4000$ & 0.0148 & 0.0849 & 0.000625 \\
$1 / 8000$ & 0.0102 & 0.0357 & 0.000358 \\
\hline
\end{tabular}

We observe a convergence rate of approximatively 1 for the variables which are continuous at the contact discontinuity $(p$ and $u$ ) and $1 / 2$ for the other ones (in the table, only $\rho$ ). Since, as explained above, the error essentially lies at the jumps of the solution, it means that a shock is captured in approximatively the same number of cells, for any of the meshes used in this test; by a simple computation, this implies that the corrective term $\left(S_{K}\right)_{K \in \mathcal{M}}$ does not tend to zero.

\section{Conclusion, Perspectives}

In this work, we studied staggered implicit and semi-implicit schemes which had been found earlier to be very efficient for viscous compressible flows [14-16]. We were able to show here that they are also well adapted 


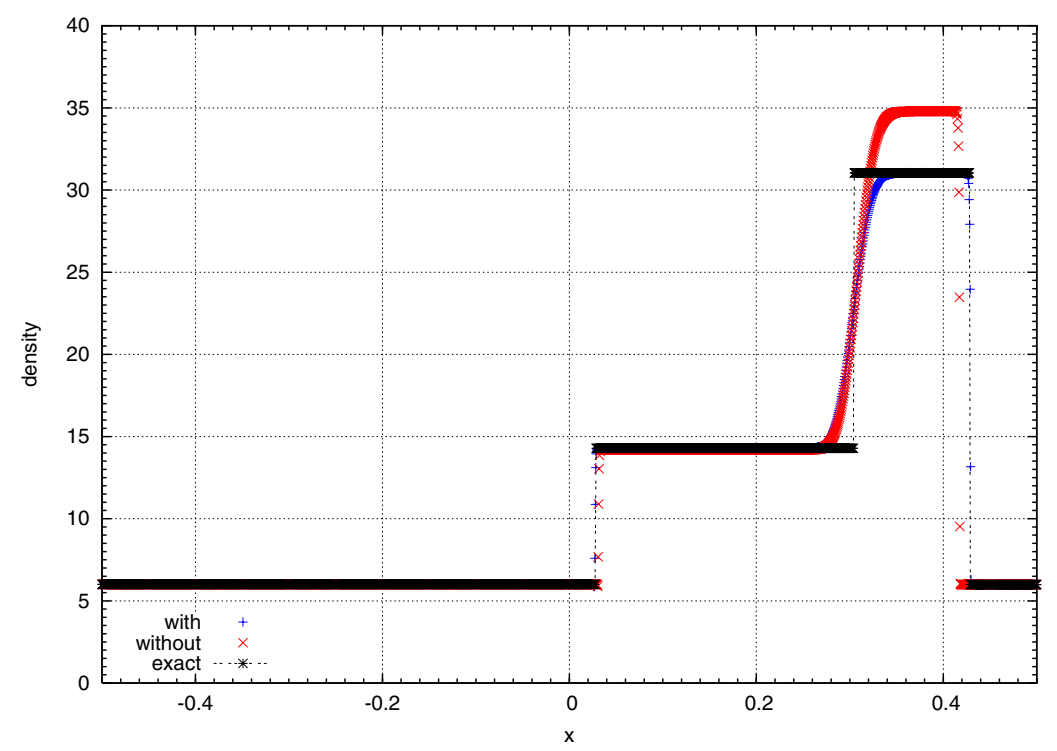

Figure 2. Test 5 of [63], Chapter 4 - Density obtained with $n=2000$ cells, with and without corrective source terms, and analytical solution.
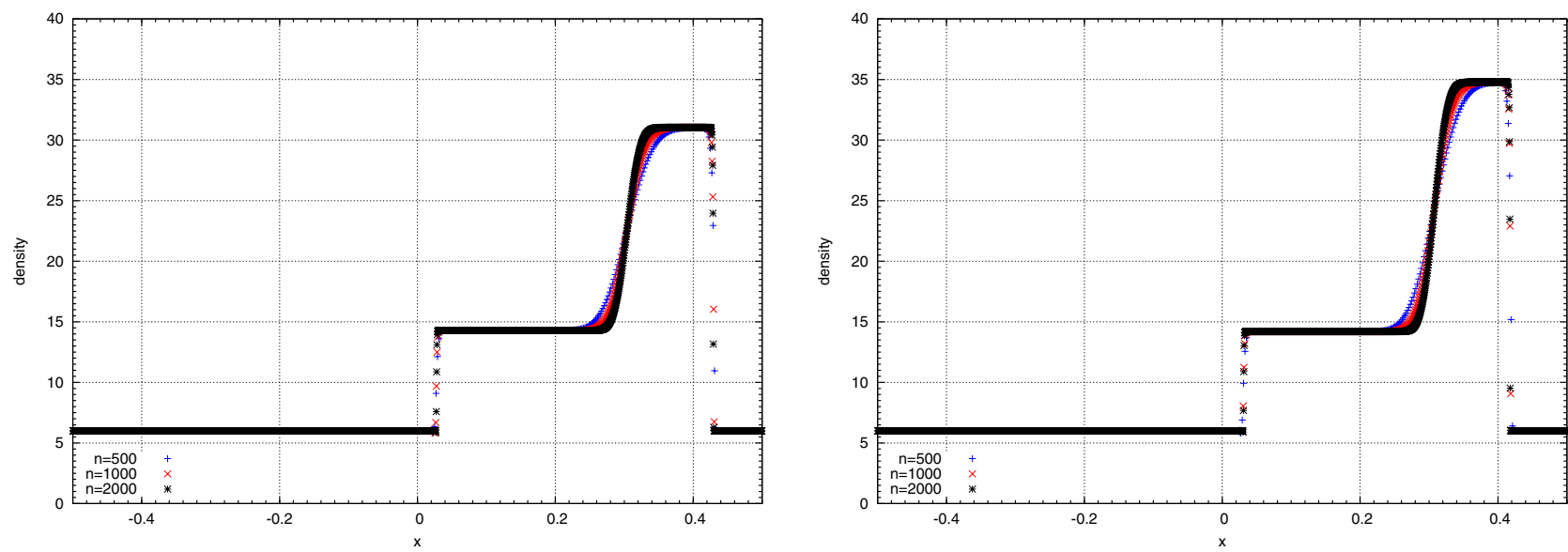

Figure 3. Test 5 of ([63], Chap. 4) - Density obtained with various meshes, with (left) and without (right) corrective source terms.

to the shallow water equations or barotropic Euler equations. We also showed that, with a careful discretization of the internal energy equation, they are again an efficient choice for the full Euler equations.

There are several open questions under study at the present time or that will be in the near future:

- A proof of the consistency of the scheme in the multidimensional case is underway: there is a real difficulty in going from the 1D case that we studied here in Sections 3.1.3, 3.2.3 and 4.3 to the multidimensional case, due to the intricate definition of the nonlinear convection term in the momentum balance equation, which makes complex the passage to the limit in this term. This difficulty has been adressed both for the fluxes defined on unstructured meshes, with the Crouzeix-Raviart and Rannacher-Turek finite element unknowns [42], and in the MAC case [29]. 

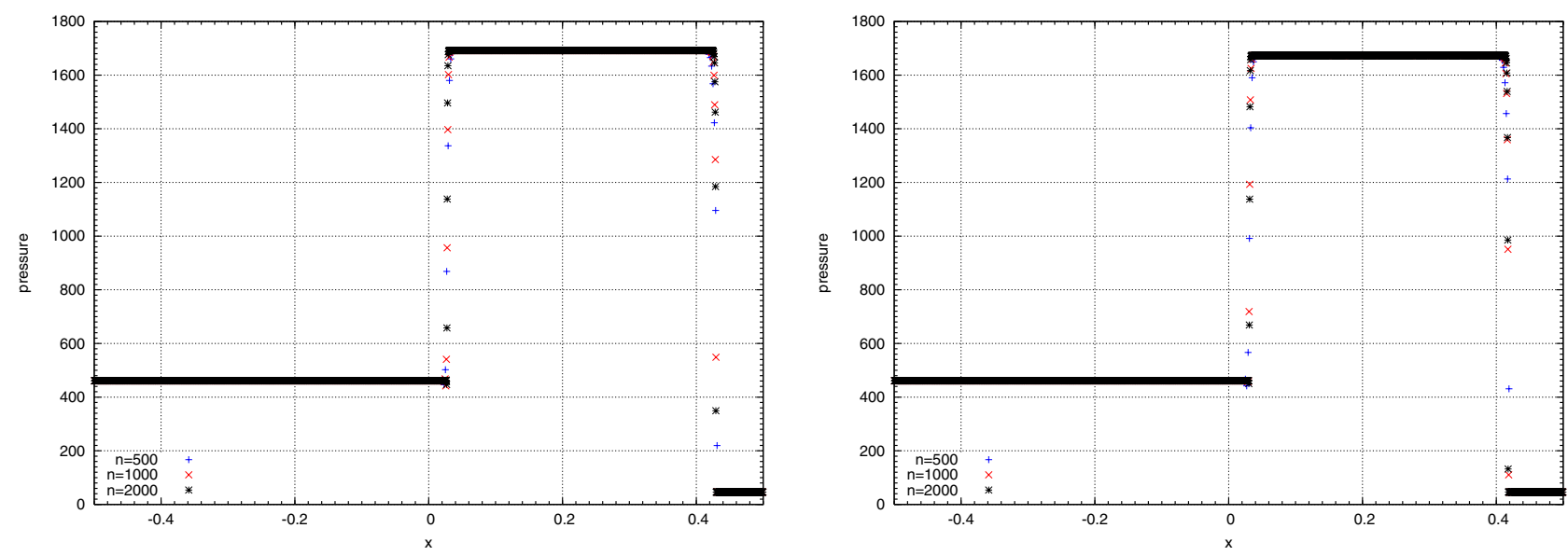

Figure 4. Test 5 of ([63], Chap. 4) - pressure obtained with various meshes, with (left) and without (right) corrective source terms.

- The consistency of the scheme with the entropy condition in the case of the full Euler equation is an open question, although the numerical results suggest that this is true. We shall continue to investigate this important issue.

- Comparisons are currently being performed, for the proposed pressure correction scheme, between the present staggered discretization and a colocated version (density, pressure and velocity unknowns all in the cells), for the Euler equations [62].

- An explicit version of this scheme has been studied both for the shallow water and Euler equations [30]. For this time discretization, higher order schemes using a MUSCL technique [56] have been studied and implemented [17].

- Finally, setting $\rho$ to a constant in the pressure correction scheme yields the usual projection scheme for the incompressible Euler (or Navier-Stokes) equations. A natural question is therefore to know whether the scheme is indeed asymptotic preserving: does the approximate solution tend to the incompressible solution as the Mach number tends to 0 ? This question should be addressed in the near future.

\section{Appendix A. Some Results Associated WITH FINITE VOLUME CONVECTION OPERATORS}

We gather in this section some results concerning the finite volume discretization of the two convection operators which appear in the Navier-Stokes equations:

- the convection operator $\mathcal{C}$ appearing in the mass balance, which reads, at the continuous level,

$$
\rho \mapsto \mathcal{C}(\rho)=\partial_{t} \rho+\operatorname{div}(\rho \boldsymbol{u}),
$$

where $\boldsymbol{u}$ stands for a given velocity field (which is not assumed to satisfy any divergence constraint),

- the convection operator $\mathcal{C}_{\rho}$ appearing in the momentum and energy balances, which reads, in the continuous setting,

$$
z \mapsto \mathcal{C}_{\rho}(z)=\partial_{t}(\rho z)+\operatorname{div}(\rho z \boldsymbol{u}),
$$

where $\rho$ (resp. $\boldsymbol{u}$ ) stands for a given scalar (resp. vector) field; we wish to obtain some property of $\mathcal{C}_{\rho}$ under the assumption that $\rho$ and $\boldsymbol{u}$ satisfy a mass balance equation, i.e. $\partial_{t} \rho+\operatorname{div}(\rho \boldsymbol{u})=0$.

Multiplying these operators by functions depending on the unknown is a classical technique to obtain convection operators acting over different variables, possibly with residual terms: one may think, for instance, to the theory 
of renormalized solutions or entropy solutions for the operator $\mathcal{C}$, or, in fluid mechanics, to the derivation of the so-called kinetic energy transport identity for the operator $\mathcal{C}_{\rho}$, with $z$ standing for a component of the velocity. The results provided in this section are the discrete analogs of these properties.

We begin with a property of the convection operator $\mathcal{C}$ defined by (A.1); at the continuous level, this property may be formally obtained as follows. Let $\psi$ be a regular function from $(0,+\infty)$ to $\mathbb{R}$; then:

$$
\psi^{\prime}(\rho) \mathcal{C}(\rho)=\psi^{\prime}(\rho) \partial_{t}(\rho)+\psi^{\prime}(\rho) \boldsymbol{u} \cdot \nabla \rho+\psi^{\prime}(\rho) \rho \operatorname{div} \boldsymbol{u}=\partial_{t}(\psi(\rho))+\boldsymbol{u} \cdot \boldsymbol{\nabla} \psi(\rho)+\rho \psi^{\prime}(\rho) \operatorname{div} \boldsymbol{u}
$$

adding and subtracting $\psi(\rho) \operatorname{div} \boldsymbol{u}$ yields:

$$
\psi^{\prime}(\rho) \mathcal{C}(\rho)=\partial_{t}(\psi(\rho))+\operatorname{div}(\psi(\rho) \boldsymbol{u})+\left(\rho \psi^{\prime}(\rho)-\psi(\rho)\right) \operatorname{div} \boldsymbol{u} .
$$

This computation is of course completely formal and only valid for regular functions $\rho$ and $\boldsymbol{u}$. The following lemma states a discrete analogue to (A.3), and its proof follows the formal computation which we just described.

Lemma A.1. Let $P$ be a polygonal (resp. polyhedral) bounded set of $\mathbb{R}^{2}$ (resp. $\mathbb{R}^{3}$ ), and let $\mathcal{E}(P)$ be the set of its edges (resp. faces). Let $\psi$ be a continuously differentiable function defined over $(0,+\infty)$. Let $\rho_{P}^{*}>0, \rho_{P}>0$, $\delta t>0$; consider three families $\left(\rho_{\eta}\right)_{\eta \in \mathcal{E}(P)} \subset \mathbb{R}_{+} \backslash\{0\},\left(V_{\eta}\right)_{\eta \in \mathcal{E}(P)} \subset \mathbb{R}$ and $\left(F_{\eta}\right)_{\eta \in \mathcal{E}(P)} \subset \mathbb{R}$ such that

$$
\forall \eta \in \mathcal{E}(P), \quad F_{\eta}=\rho_{\eta} V_{\eta},
$$

and define:

$$
\begin{aligned}
R_{P, \delta t}= & {\left[\frac{|P|}{\delta t}\left(\rho_{P}-\rho_{P}^{*}\right)+\sum_{\eta \in \mathcal{E}(P)} F_{\eta}\right] \psi^{\prime}\left(\rho_{P}\right) } \\
& -\left[\frac{|P|}{\delta t}\left(\psi\left(\rho_{P}\right)-\psi\left(\rho_{P}^{*}\right)\right)+\sum_{\eta \in \mathcal{E}(P)} \psi\left(\rho_{\eta}\right) V_{\eta}+\left(\rho_{P} \psi^{\prime}\left(\rho_{P}\right)-\psi\left(\rho_{P}\right)\right) \sum_{\eta \in \mathcal{E}(P)} V_{\eta}\right]
\end{aligned}
$$

Then

(i) If $\psi$ is convex and $\rho_{\eta}=\rho_{P}$ whenever $V_{\eta}>0$, then $R_{P, \delta t} \geq 0$.

(ii) If $\psi$ is twice continuously differentiable then

$$
R_{P, \delta t}=\frac{1}{2} \frac{|P|}{\delta t} \psi^{\prime \prime}\left(\bar{\rho}_{P}\right)\left(\rho_{P}-\rho_{P}^{*}\right)^{2}-\frac{1}{2} \sum_{\eta \in \mathcal{E}(P)} V_{\eta} \psi^{\prime \prime}\left(\bar{\rho}_{\eta}\right)\left(\rho_{\eta}-\rho_{P}\right)^{2},
$$

with $\bar{\rho}_{P} \in\left[\min \left(\rho_{P}, \rho_{P}^{*}\right), \max \left(\rho_{P}, \rho_{P}^{*}\right)\right]$ and $\forall \eta \in \mathcal{E}(P), \bar{\rho}_{\eta} \in\left[\min \left(\rho_{\eta}, \rho_{P}\right), \max \left(\rho_{\eta}, \rho_{P}\right)\right]$.

Proof. We have:

$$
\begin{aligned}
{\left[\frac{|P|}{\delta t}\left(\rho_{P}-\rho_{P}^{*}\right)+\sum_{\eta \in \mathcal{E}(P)} F_{\eta}\right] \psi^{\prime}\left(\rho_{P}\right)=} & \frac{|P|}{\delta t}\left(\rho_{P}-\rho_{P}^{*}\right) \psi^{\prime}\left(\rho_{P}\right)+\sum_{\eta \in \mathcal{E}(P)} \psi\left(\rho_{\eta}\right) V_{\eta} \\
& +\sum_{\eta \in \mathcal{E}(P)}\left[\rho_{\eta} \psi^{\prime}\left(\rho_{P}\right)-\psi\left(\rho_{\eta}\right)\right] V_{\eta}
\end{aligned}
$$

so the remainder term $R_{P, \delta t}$ reads $R_{P, \delta t}=\frac{|P|}{\delta t} r_{P}+\sum_{\eta \in \mathcal{E}(P)} V_{\eta} r_{\eta}$, with:

$$
r_{P}=\left(\rho_{P}-\rho_{P}^{*}\right) \psi^{\prime}\left(\rho_{P}\right)-\left[\psi\left(\rho_{P}\right)-\psi\left(\rho_{P}^{*}\right)\right], \quad r_{\eta}=\rho_{\eta} \psi^{\prime}\left(\rho_{P}\right)-\psi\left(\rho_{\eta}\right)-\left[\rho_{P} \psi^{\prime}\left(\rho_{P}\right)-\psi\left(\rho_{P}\right)\right] .
$$


If the function $\psi$ is convex, $r_{P}$ is non-negative while $r_{\eta}$ is non-positive (and vanishes if $\rho_{\eta}=\rho_{P}$ ). If $\psi$ is twice continuously differentiable, a Taylor expansion gives that:

$$
\begin{aligned}
& \left(\rho_{P}-\rho_{P}^{*}\right) \psi^{\prime}\left(\rho_{P}\right)=\psi\left(\rho_{P}\right)-\psi\left(\rho_{P}^{*}\right)+\frac{1}{2} \psi^{\prime \prime}\left(\bar{\rho}_{P}\right)\left(\rho_{P}-\rho_{P}^{*}\right)^{2}, \\
& \rho_{\eta} \psi^{\prime}\left(\rho_{P}\right)-\psi\left(\rho_{\eta}\right)=\rho_{P} \psi^{\prime}\left(\rho_{P}\right)-\psi\left(\rho_{P}\right)-\frac{1}{2} \psi^{\prime \prime}\left(\bar{\rho}_{\eta}\right)\left(\rho_{\eta}-\rho_{P}\right)^{2},
\end{aligned}
$$

with $\bar{\rho}_{P} \in\left[\min \left(\rho_{P}, \rho_{P}^{*}\right), \max \left(\rho_{P}, \rho_{P}^{*}\right)\right]$ and for any $\eta \in \mathcal{E}(P), \bar{\rho}_{\eta} \in\left[\min \left(\rho_{\eta}, \rho_{P}\right), \max \left(\rho_{\eta}, \rho_{P}\right)\right]$; hence the result.

We now turn to the momentum convection operator $\mathcal{C}_{\rho}$ defined by (A.2); formally, using twice the assumption $\partial_{t} \rho+\operatorname{div}(\rho \boldsymbol{u})=0$ yields:

$$
\begin{aligned}
\psi^{\prime}(z) \mathcal{C}_{\rho}(z) & =\psi^{\prime}(z)\left[\partial_{t}(\rho z)+\operatorname{div}(\rho z \boldsymbol{u})\right]=\psi^{\prime}(z) \rho\left[\partial_{t} z+\boldsymbol{u} \cdot \nabla z\right] \\
& =\rho\left[\partial_{t} \psi(z)+\boldsymbol{u} \cdot \nabla \psi(z)\right]=\partial_{t}(\rho \psi(z))+\operatorname{div}(\rho \psi(z) \boldsymbol{u}) .
\end{aligned}
$$

Taking for $z$ a component of the velocity field, this relation is the central argument used to derive the kinetic energy balance. The following lemma states a discrete counterpart of this identity.

Lemma A.2. Let $P$ be a polygonal (resp. polyhedral) bounded set of $\mathbb{R}^{2}$ (resp. $\mathbb{R}^{3}$ ) and let $\mathcal{E}(P)$ be the set of its edges (resp. faces). Let $\rho_{P}^{*}>0, \rho_{P}>0, \delta t>0$, and $\left(F_{\eta}\right)_{\eta \in \mathcal{E}(P)} \subset \mathbb{R}$ be such that

$$
\frac{|P|}{\delta t}\left(\rho_{P}-\rho_{P}^{*}\right)+\sum_{\eta \in \mathcal{E}(P)} F_{\eta}=0 .
$$

Let $\psi$ be a continuously differentiable function defined over $(0,+\infty)$. For $u_{P}^{*} \in \mathbb{R}, u_{P} \in \mathbb{R}$ and $\left(u_{\eta}\right)_{\eta \in \mathcal{E}(P)} \subset \mathbb{R}$ let us define:

$$
\begin{aligned}
R_{P, \delta t} & =\left[\frac{|P|}{\delta t}\left(\rho_{P} u_{P}-\rho_{P}^{*} u_{P}^{*}\right)+\sum_{\eta \in \mathcal{E}(P)} F_{\eta} u_{\eta}\right] \psi^{\prime}\left(u_{P}\right) \\
& -\left[\frac{|P|}{\delta t}\left[\rho_{P} \psi\left(u_{P}\right)-\rho_{P}^{*} \psi\left(u_{P}^{*}\right)\right]+\sum_{\eta \in \mathcal{E}(P)} F_{\eta} \psi\left(u_{\eta}\right)\right] .
\end{aligned}
$$

Then:

(i) If $\psi$ is convex and $u_{\eta}=u_{P}$ whenever $F_{\eta}>0$, then $R_{P, \delta t} \geq 0$.

(ii) If $\psi$ is twice continuously differentiable, then

$$
R_{P, \delta t}=\frac{1}{2} \frac{|P|}{\delta t} \rho_{P}^{*} \psi^{\prime \prime}\left(\bar{u}_{P}\right)\left(u_{P}-u_{P}^{*}\right)^{2}-\frac{1}{2} \sum_{\eta \in \mathcal{E}(P)} F_{\eta} \psi^{\prime \prime}\left(\bar{u}_{\eta}\right)\left(u_{\eta}-u_{P}\right)^{2},
$$

with, $\bar{u}_{P} \in\left[\min \left(u_{P}, u_{P}^{*}\right), \max \left(u_{P}, u_{P}^{*}\right)\right]$ and, $\forall \eta \in \mathcal{E}(P), \bar{u}_{\eta} \in\left[\min \left(u_{\eta}, u_{P}\right), \max \left(u_{\eta}, u_{P}\right)\right]$.

(iii) As a consequence of (ii), for $\psi$ defined by $\psi(s)=s^{2} / 2$, and $\forall \eta \in \mathcal{E}(P), u_{\eta}$ such that $u_{\eta}=\left(u_{P}+u_{P_{\eta}}\right) / 2$ (this is simply obtained by defining $u_{P_{\eta}}=2 u_{\eta}-u_{P}$ ), we get the following identity:

$$
\left[\frac{|P|}{\delta t}\left(\rho_{P} u_{P}-\rho_{P}^{*} u_{P}^{*}\right)+\sum_{\eta \in \mathcal{E}(P)} F_{\eta} u_{\eta}\right] u_{P}=\frac{1}{2} \frac{|P|}{\delta t}\left[\rho_{P} u_{P}^{2}-\rho_{P}^{*}\left(u_{P}^{*}\right)^{2}\right]+\frac{1}{2} \sum_{\eta \in \mathcal{E}(P)} F_{\eta} u_{P} u_{P_{\eta}}+\tilde{R}_{P, \delta t},
$$

with

$$
\tilde{R}_{P, \delta t}=\frac{1}{2} \frac{|P|}{\delta t} \rho_{P}^{*}\left(u_{P}-u_{P}^{*}\right)^{2},
$$


Proof. Let $T_{P}$ be defined by:

$$
T_{P}=\left[\frac{|P|}{\delta t}\left(\rho_{P} u_{P}-\rho_{P}^{*} u_{P}^{*}\right)+\sum_{\eta \in \mathcal{E}(P)} F_{\eta} u_{\eta}\right] \psi^{\prime}\left(u_{P}\right) .
$$

Using equation (A.6), we obtain:

$$
T_{P}=\left[\frac{|P|}{\delta t} \rho_{P}^{*}\left(u_{P}-u_{P}^{*}\right)+\sum_{\eta \in \mathcal{E}(P)} F_{\eta}\left(u_{\eta}-u_{P}\right)\right] \psi^{\prime}\left(u_{P}\right) .
$$

We now define the remainder terms $r_{P}$ and $\left(r_{\eta}\right)_{\eta \in \mathcal{E}(P)}$ by:

$$
r_{P}=\left(u_{P}-u_{P}^{*}\right) \psi^{\prime}\left(u_{P}\right)-\left[\psi\left(u_{P}\right)-\psi\left(u_{P}^{*}\right)\right], \quad r_{\eta}=\left(u_{P}-u_{\eta}\right) \psi^{\prime}\left(u_{P}\right)-\left[\psi\left(u_{P}\right)-\psi\left(u_{\eta}\right)\right] .
$$

With these notations, we get:

$$
T_{P}=\frac{|P|}{\delta t} \rho_{P}^{*}\left[\psi\left(u_{P}\right)-\psi\left(u_{P}^{*}\right)\right]+\sum_{\eta \in \mathcal{E}(P)} F_{\eta}\left[\psi\left(u_{\eta}\right)-\psi\left(u_{P}\right)\right]+\frac{|P|}{\delta t} \rho_{P}^{*} r_{P}-\sum_{\eta \in \mathcal{E}(P)} F_{\eta} r_{\eta} .
$$

Using equation (A.6) once again, we have:

$$
T_{P}=\frac{|P|}{\delta t}\left[\rho_{P} \psi\left(u_{P}\right)-\rho_{P}^{*} \psi\left(u_{P}^{*}\right)\right]+\sum_{\eta \in \mathcal{E}(P)} F_{\eta} \psi\left(u_{\eta}\right)+\frac{|P|}{\delta t} \rho_{P}^{*} r_{P}-\sum_{\eta \in \mathcal{E}(P)} F_{\eta} r_{\eta},
$$

and thus:

$$
R_{P, \delta t}=\frac{|P|}{\delta t} \rho_{P}^{*} r_{P}-\sum_{\eta \in \mathcal{E}(P)} F_{\eta} r_{\eta}
$$

If $\psi$ is convex, the remainder terms $r_{P}$ and $\left(r_{\eta}\right)_{\eta \in \mathcal{E}(P)}$ are non-negative, and if $u_{\eta}=u_{P}, r_{\eta}=0$; hence, if we suppose that $u_{\eta}=u_{P}$ when $F_{\eta} \geq 0$, then $R_{P, \delta t} \geq 0$. If $\psi$ is twice continuously differentiable, a Taylor expansion yields:

$$
r_{P}=\frac{1}{2} \psi^{\prime \prime}\left(\bar{u}_{P}\right)\left(u_{p}-u_{p}^{*}\right)^{2}, \quad r_{\eta}=\frac{1}{2} \psi^{\prime \prime}\left(\bar{u}_{\eta}\right)\left(u_{\eta}-u_{p}\right)^{2}
$$

with $\bar{u}_{P} \in\left[\min \left(u_{P}, u_{P}^{*}\right), \max \left(u_{P}, u_{P}^{*}\right)\right]$ and, $\forall \eta \in \mathcal{E}(P), \bar{u}_{\eta} \in\left[\min \left(u_{\eta}, u_{P}\right), \max \left(u_{\eta}, u_{P}\right)\right]$. Thus (ii) holds. The assertion (iii) is then a direct consequence of (ii).

\section{REFERENCES}

[1] G. Ansanay-Alex, F. Babik, J.-C. Latché and D. Vola, An $\mathrm{L}^{2}$-stable approximation of the Navier-Stokes convection operator for low-order non-conforming finite elements. Int. J. Numer. Methods Fluids 66 (2011) 555-580.

[2] F. Archambeau, J.-M. Hérard and J. Laviéville, Comparative study of pressure-correction and Godunov-type schemes on unsteady compressible cases. Comput. Fluids 38 (2009) 1495-1509.

[3] R. Berry, Notes on PCICE method: simplification, generalization and compressibility properties. J. Comput. Phys. 215 (2006) $6-11$.

[4] H. Bijl and P. Wesseling, A unified method for computing incompressible and compressible flows in boundary-fitted coordinates. J. Comput. Phys. 141 (1998) 153-173.

[5] $\mathrm{CALIF}^{3} \mathrm{~S}$. A software components library for the computation of reactive turbulent flows. Available on https://gforge.irsn.fr/gf/project/isis.

[6] V. Casulli and D. Greenspan, Pressure method for the numerical solution of transient, compressible fluid flows. Int. J. Numer. Methods Fluids 4 (1984) 1001-1012.

[7] A. Chorin, Numerical solution of the Navier-Stokes equations. Math. Comput. 22 (1968) 745-762.

[8] P.G. Ciarlet, Basic error estimates for elliptic problems, in vol. II of Handb. Numer. Anal. Edited by P. Ciarlet and J. Lions. North Holland (1991) 17-351. 
[9] M. Crouzeix and P. Raviart, Conforming and nonconforming finite element methods for solving the stationary Stokes equations. RAIRO Série Rouge 7 (1973) 33-75.

[10] I. Demirdžić, v. Lilek and M. Perić, A collocated finite volume method for predicting flows at all speeds. Int. J. Numer. Methods Fluids 16 (1993) 1029-1050.

[11] R. Eymard, T. Gallouët and R. Herbin, Finite volume methods, in vol. VII of Handb. Numer. Anal. Edited by P. Ciarlet and J. Lions. North Holland (2000) 713-1020.

[12] R. Eymard, T. Gallouët, R. Herbin and J.-C. Latché, Convergence of the MAC scheme for the compressible Stokes equations. SIAM J. Numer. Anal. 48 (2010) 2218-2246.

[13] E. Feireisl, Dynamics of Viscous Compressible Flows. In vol. 26 of Oxford Lect. Ser. Math. Appl. Oxford University Press (2004).

[14] T. Gallouët, L. Gastaldo, R. Herbin and J.-C. Latché, An unconditionally stable pressure correction scheme for compressible barotropic Navier-Stokes equations. Math. Model. Numer. Anal. 42 (2008) 303-331.

[15] L. Gastaldo, R. Herbin, W. Kheriji, C. Lapuerta and J.-C. Latché, Staggered discretizations, pressure correction schemes and all speed barotropic flows, in Finite Volumes for Complex Applications VI - Problems and Perspectives Vol. 2, - Prague, Czech Republic (2011) 39-56.

[16] L. Gastaldo, R. Herbin and J.-C. Latché, A discretization of phase mass balance in fractional step algorithms for the drift-flux model. IMA J. Numer. Anal. 3 (2011) 116-146.

[17] L. Gastaldo, R. Herbin, J.-C. Latché and N. Therme, Explicit high order staggered schemes for the Euler equations (2014).

[18] D. Grapsas, R. Herbin, W. Kheriji and J.-C. Latché, An unconditionally stable pressure correction scheme for the compressible Navier-Stokes equations. Submitted (2014).

[19] J. Guermond, P. Minev and J. Shen, An overview of projection methods for incompressible flows. Comput. Methods Appl. Mech. Engrg. 195 (2006) 6011-6045.

[20] J. Guermond and R. Pasquetti, Entropy-based nonlinear viscosity for Fourier approximations of conservation laws. C.R. Acad. Sci. Paris - Série I - Analyse Numérique 346 (2008) 801-806.

[21] J. Guermond, R. Pasquetti and B. Popov, Entropy viscosity method for nonlinear conservation laws. J. Comput. Phys. 230 (2011) 4248-4267.

[22] J.-L. Guermond and L. Quartapelle, A projection FEM for variable density incompressible flows. J. Comput. Phys. 165 (2000) $167-188$.

[23] F. Harlow and A. Amsden, Numerical calculation of almost incompressible flow. J. Comput. Phys. 3 (1968) $80-93$.

[24] F. Harlow and A. Amsden, A numerical fluid dynamics calculation method for all flow speeds. J. Comput. Phys. 8 (1971) 197-213.

[25] F. Harlow and J. Welsh, Numerical calculation of time-dependent viscous incompressible flow of fluid with free surface. Phys. Fluids 8 (1965) 2182-2189.

[26] R. Herbin, W. Kheriji and J.-C. Latché, Staggered schemes for all speed flows. ESAIM Proc. 35 (2012) $22-150$.

[27] R. Herbin, W. Kheriji and J.-C. Latché, Pressure correction staggered schemes for barotropic monophasic and two-phase flows. Comput. Fluids 88 (2013) 524-542.

[28] R. Herbin and J.-C. Latché, Kinetic energy control in the MAC discretization of the compressible Navier-Stokes equations. Int. J. Finites Volumes 7 (2010).

[29] R. Herbin, J.-C. Latché and K. Mallem, Convergence of the MAC scheme for the steady-state incompressible Navier-Stokes equations on non-uniform grids. Proc. of Finite Volumes for Complex Applications VII - Problems and Perspectives, Berlin, Germany (2014).

[30] R. Herbin, J.-C. Latché and T. Nguyen, An explicit staggered scheme for the shallow water and Euler equations. Submitted (2013).

[31] R. Herbin, J.-C. Latché and T. Nguyen, Explicit staggered schemes for the compressible euler equations. ESAIM Proc. 40 (2013) 83-102.

[32] B. Hjertager, Computer simulation of reactive gas dynamics. Vol. 5 of Modeling, Identification and Control (1985) $211-236$.

[33] Y. Hou and K. Mahesh, A robust, colocated, implicit algorithm for direct numerical simulation of compressible, turbulent flows. J. Comput. Phys. 205 (2005) 205-221.

[34] R. Issa, Solution of the implicitly discretised fluid flow equations by operator splitting. J. Comput. Phys. 62 (1985) 40-65.

[35] R. Issa, A. Gosman and A. Watkins, The computation of compressible and incompressible recirculating flows by a non-iterative implicit scheme. J. Comput. Phys. 62 (1986) 66-82.

[36] R. Issa and M. Javareshkian, Pressure-based compressible calculation method utilizing total variation diminishing schemes. AIAA J. 36 (1998) 1652-1657.

[37] S. Kadioglu, M. Sussman, S. Osher, J. Wright and M. Kang, A second order primitive preconditioner for solving all speed multi-phase flows. J. Comput. Phys. 209 (2005) 477-503.

[38] K. Karki and S. Patankar, Pressure based calculation procedure for viscous flows at all speeds in arbitrary configurations. AIAA J. 27 (1989) 1167-1174.

[39] M. Kobayashi and J. Pereira. Characteristic-based pressure correction at all speeds. AIAA J. 34 (1996) 272-280.

[40] A. Kurganov and Y. Liu, New adaptative artificial viscosity method for hyperbolic systems of conservation laws. J. Comput. Phys. 231 (2012) 8114-8132.

[41] N. Kwatra, J. Su, J. Grétarsson and R. Fedkiw, A method for avoiding the acoustic time step restriction in compressible flow. J. Comput. Phys. 228 (2009) 4146-4161. 
[42] J.-C. Latché and K. Saleh, A convergent staggered scheme for variable density incompressible Navier-Stokes equations. Submitted (2014).

[43] F.-S. Lien, A pressure-based unstructured grid method for all-speed flows. Int. J. Numer. Methods Fluids 33 (2000) 355-374.

[44] P.-L. Lions, Mathematical Topics in Fluid Mechanics - Volume 2 - Compressible Models. Vol. 10 of Oxford Lect. Ser. Math. Appl. Oxford University Press (1998).

[45] A. Majda and J. Sethian. The derivation and numerical solution of the equations for zero Mach number solution. Combust. Sci. Techn. 42 (1985) 185-205.

[46] R. Martineau and R. Berry, The pressure-corrected ICE finite element method for compressible flows on unstructured meshes. J. Comput. Phys. 198 (2004) 659-685.

[47] J. McGuirk and G. Page, Shock capturing using a pressure-correction method. AIAA J. 28 (1990) 1751-1757.

[48] F. Moukalled and M. Darwish, A high-resolution pressure-based algorithm for fluid flow at all speeds. J. Comput. Phys. 168 (2001) 101-133.

[49] V. Moureau, C. Bérat and H. Pitsch, An efficient semi-implicit compressible solver for large-eddy simulations. J. Comput. Phys. 226 (2007) 1256-1270.

[50] K. Nerinckx, J. Vierendeels and E. Dick, Mach-uniformity through the coupled pressure and temperature correction algorithm. J. Comput. Phys. 206 (2005) 597-623.

[51] K. Nerinckx, J. Vierendeels and E. Dick. A Mach-uniform algorithm: coupled versus segregated approach. J. Comput. Phys. 224 (2007) 314-331.

[52] P. Nithiarasu, R. Codina and O. Zienkiewicz, The Characteristic-Based Split (CBS) scheme - A unified approach to fluid dynamics. Int. J. Numer. Methods Engrg. 66 (2006) 1514-1546.

[53] A. Novotný and I. Straškraba, Introduction to the Mathematical Theory of Compressible Flow. Vol. 27 of Oxford Lect. Ser. Math. Appl. Oxford University Press (2004).

[54] G. Patnaik, R. Guirguis, J. Boris and E. Oran, A barely implicit correction for flux-corrected transport. J. Comput. Phys. 71 (1987) 1-20.

[55] PELICANS, Collaborative development environment. Available on https://gforge.irsn.fr/gf/project/pelicans.

[56] L. Piar, F. Babik, R. Herbin and J.-C. Latché, A formally second order cell centered scheme for convection-diffusion equations on unstructured nonconforming grids. Int. J. Numer. Methods Fluids $\mathbf{7 1}$ (2013) 873-890.

[57] E. Politis and K. Giannakoglou, A pressure-based algorithm for high-speed turbomachinery flows. Int. J. Numer. Methods Fluids 25 (1997) 63-80.

[58] R. Rannacher and S. Turek. Simple nonconforming quadrilateral Stokes element. Numer. Methods Partial Differ. Equ. 8 (1992) 97-111.

[59] E. Sewall and D. Tafti, A time-accurate variable property algorithm for calculating flows with large temperature variations. Comput. Fluids 37 (2008) 51-63.

[60] R. Temam, Sur l'approximation de la solution des équations de Navier-Stokes par la méthode des pas fractionnaires II. Arch. Rat. Mech. Anal. 33 (1969) 377-385.

[61] S. Thakur and J. Wright, A multiblock operator-splitting algorithm for unsteady flows at all speeds in complex geometries. Int. J. Numer. Methods Fluids 46 (2004) 383-413.

[62] N. Therme and Z. Chady, Comparison of consistent explicit schemes on staggered and colocated meshes (2014).

[63] E. Toro, Riemann solvers and numerical methods for fluid dynamics - A practical introduction, 3rd edition. Springer (2009).

[64] D. Van der Heul, C. Vuik and P. Wesseling, Stability analysis of segregated solution methods for compressible flow. Appl. Numer. Math. 38 (2001) 257-274.

[65] D. Van der Heul, C. Vuik and P. Wesseling. A conservative pressure-correction method for flow at all speeds. Comput. Fluids 32 (2003) 1113-1132.

[66] J. Van Dormaal, G. Raithby and B. McDonald, The segregated approach to predicting viscous compressible fluid flows. Trans. ASME 109 (1987) 268-277.

[67] D. Vidović, A. Segal and P. Wesseling, A superlinearly convergent Mach-uniform finite volume method for the Euler equations on staggered unstructured grids. J. Comput. Phys. 217 (2006) 277-294.

[68] C. Wall, C. Pierce and P. Moin, A semi-implicit method for resolution of acoustic waves in low Mach number flows. J. Comput. Phys. 181 (2002) 545-563.

[69] I. Wenneker, A. Segal and P. Wesseling, A Mach-uniform unstructured staggered grid method. Int. J. Numer. Methods Fluids 40 (2002) 1209-1235.

[70] C. Xisto, J. Páscoa, P. Oliveira and D. Nicolini, A hybrid pressure-density-based algorithm for the Euler equations at all Mach number regimes. Int. J. Numer. Methods Fluids, online (2011).

[71] S. Yoon and T. Yabe, The unified simulation for incompressible and compressible flow by the predictor-corrector scheme based on the CIP method. Comput. Phys. Commun. 119 (1999) 149-158.

[72] O. Zienkiewicz and R. Codina, A general algorithm for compressible and incompressible flow - Part I. The split characteristicbased scheme. Int. J. Numer. Methods Fluids 20 (1995) 869-885. 